\title{
SECURING A HEALTH INFORMATION SYSTEM WITH A GOVERNMENT ISSUED DIGITAL IDENTIFICATION CARD
}

\author{
By \\ Ricardo Filipe Sousa Santos \\ A thesis submitted to \\ Faculdade de Medicina / Faculdade de Ciências \\ Universidade do Porto \\ in partial fulfilment of \\ the requirements for the degree of \\ Master of Science in Medical Informatics
}

Oporto, Portugal

September 2009

Advisors: Manuel Eduardo Correia, PhD; Luís Filipe Antunes, PhD

(C) Copyright

2009, Ricardo Filipe Sousa Santos 


\begin{abstract}
Health Information Systems (HIS) are often deployed with inadequate security solutions. Given the importance and sensitivity of this matter and to guarantee the rule of law, as well as the legal and ethical value of decisions made in a hospital setting, often dealing with life/death situations, there is an urgent need for a much better provisioning of health information systems security.

The launch of the Portuguese digital national identification smart card, the Citizen Card (CC), with strong authentication and digital signing capabilities, represents a new and very viable economic opportunity for securing a HIS and at the same time foster the creation of a much more secure national health information infrastructure. The CC is a versatile and secure card, with the latest in encryption and tamper resistance technologies, with standard support for a Public Key Infrastructure (PKI). The CC holds identification data in three different ways: printed, digital and machine readable. It substitutes five national cards, including the national health system ID card. The CC has separate authentication and digital signature keys, both issued and managed by a government certificate authority (CA).
\end{abstract}

The main issues and problems with HIS security are presented, followed by brief explorations of themes related to the CC and HIS security. The relevant card characteristics are presented and discussed, with a special emphasis on the security aspects of the card when applied into the healthcare context.

Smart cards are being deployed in healthcare everywhere around the world with highly encouraging results. Major programs have already been deployed with great success, although there are a few well identified issues that need to be addressed. The CC card has the advantage of having a PKI already in place at the national level, so the initial costs of integration testing, deployment and card management have already been taken care of by the national government. HIS advantage on this new opportunity is discussed from a technical and human perspective, and some of the major deployment problems that are likely to be encountered are presented, as well as HIS areas where the smart card crypto functionalities can be deployed with great advantage.

There are advantages, as well as some problems in using a smart card in a HIS. The CC possible contributions in this field are as an easy, inexpensive, widely deployed way of using current technology to protect HIS security, and, ultimately, patient information, while at the same time fostering the expansion and deployment of interoperable HIS. Problems identified 
include possible reluctance in broad utilization of the $\mathrm{CC}$ in a professional setting, as well as the need to change currently adopted (although insecure) workflows.

The CC may have a role in advancing Electronic Health Records, protecting widely available personal health records, allowing better allocation of health human resources, promoting telemedicine, and above all, protecting the growing health data, both in privacy and quality, in accordance with the rule of law. 


\section{Resumo}

Os sistemas de Informação na Saúde (HIS) são muitas vezes criados com soluções de segurança inadequadas. Dada a elevada importância desta informação e para que se garanta o cumprimento da lei, bem como o valor das decisões legais e éticas tomadas nos hospitais, muitas vezes lidando com situações de vida ou morte, há uma necessidade urgente para um melhor aprovisionamento de da segurança dos sistemas de informação na saúde.

A criação do cartão de identificação nacional electrónico Português, o cartão do cidadão (CC), um smart card com meios de autenticação e assinatura digitais qualificados, representa uma nova e muito viável oportunidade económica para tornar os HIS mais seguros, e simultaneamente proporcionar a criação de uma melhor infra-estrutura nacional de informação na saúde. O CC é uma cartão seguro e versátil, possuidor das melhores tecnologias de encriptação e resistência a fraude, com suporte para uma infra-estrutura de chaves públicas (PKI). O CC possui dados visíveis de três formas: Imprimidos, digitais e passíveis de leitura óptica automática (MRZ). Ele substitui cinco cartões nacionais, incluindo o cartão do serviço nacional de saúde. O CC tem pares de chaves de autenticação e assinatura diferentes, ambas emitidas e geridas pela autoridade certificadora do governo.

As principais características e problemas dos HIS são apresentados, seguidos por um breve incurso em temas relacionados com o CC e a segurança dos HIS. As características relevantes do cartão são apresentadas e discutidas, com especial ênfase nos aspectos de segurança do cartão quando aplicado num contexto de cuidados de saúde.

Os smart cards estão a ser aplicados na saúde um pouco por todo o mundo, com resultados altamente encorajadores. Alguns projectos importantes já avançaram com resultados encorajadores, ainda que persistam alguns problemas que merecem atenção. O CC tem a vantagem de possuir uma PKI em funcionamento a nível nacional, pelo que os custos iniciais de emissão de cartões, testes, e de administração dos cartões já foram suportados pelo governo. As vantagens que os HIS poderão obter com esta oportunidade são discutidas sob o ponto de vista técnico e humano, bem como alguns dos problemas importantes que poderão ser encontrados na sua aplicação, e as áreas dos HIS onde as funcionalidades criptográficas do CC serão melhor aproveitadas.

Há vantagens, bem como alguns problemas no uso de um smart card num HIS. O CC apresenta-se como uma solução fácil, de baixo custo, ubíqua, no uso da tecnologia ao nosso dispor para tornar os HIS mais seguros, e em última análise, os registos clínicos, ao mesmo 
tempo proporcionando a expansão e criação de HIS inter-operáveis. Os problemas identificados incluem a possível relutância no uso de um cartão pessoal no ambiente de trabalho, bem como na necessidade de alterar métodos correntes e enraizados (ainda que inseguros).

O CC poderá ter um papel preponderante na criação e melhoria dos registos de saúde electrónicos, protegendo, de forma segura, registos acessíveis pela internet, permitindo uma melhor gestão de recursos humanos, promovendo o uso da telemedicina, e, acima de tudo, protegendo o crescente armazenamento de informação de saúde, tanto a nível da sua privacidade como da qualidade, de acordo com as leis em vigor. 


\section{Acknowledgments}

I would like to thank Professors Manuel Eduardo Correia and Luís Filipe Antunes, for their orientation and support, the staff from the Master of Science in Medical Informatics course, for their enriching classes and Professor Altamiro da Costa Pereira, for his inspiring leadership. 


\section{Dedication}

To my ever supporting wife Patrícia and my wonderful son Francisco. 


\section{Contents}

ACKNOWLEDGMENTSS

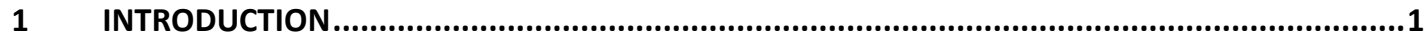

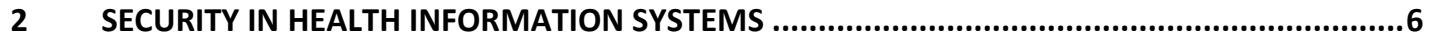

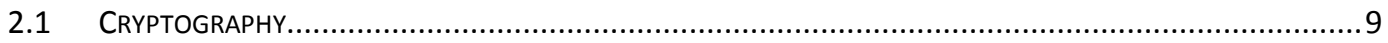

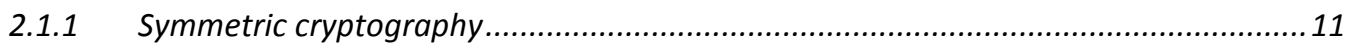

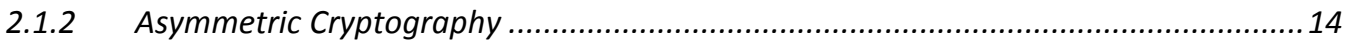

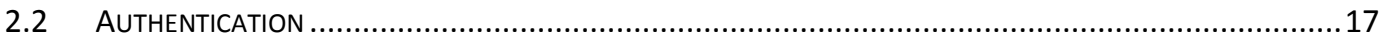

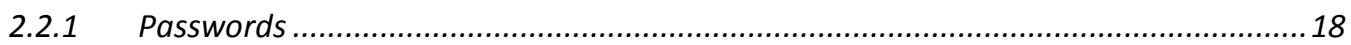

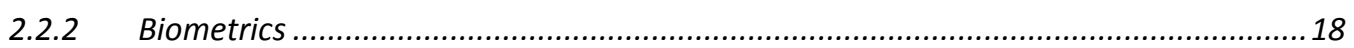

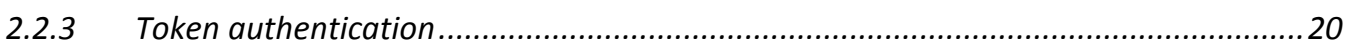

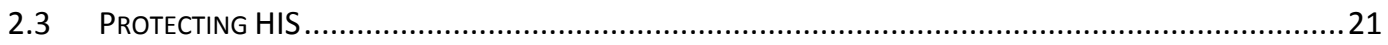

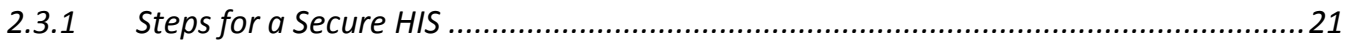

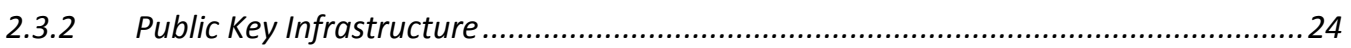

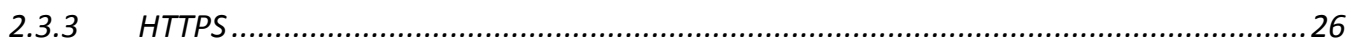

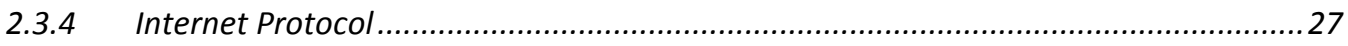

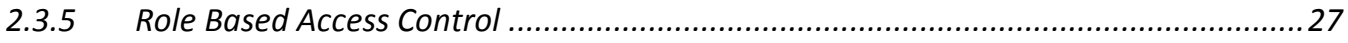

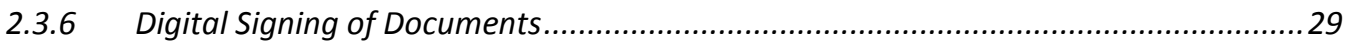

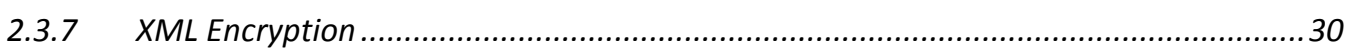

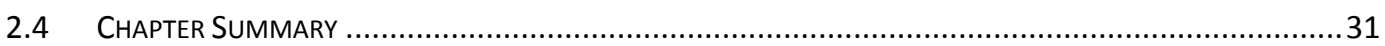

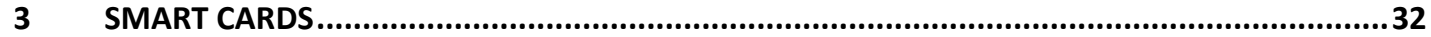

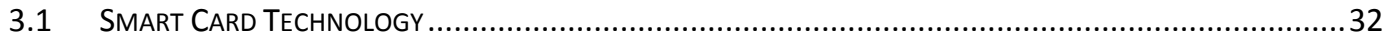

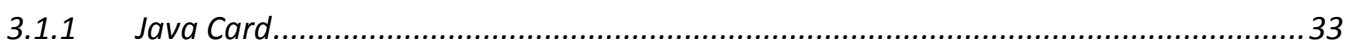

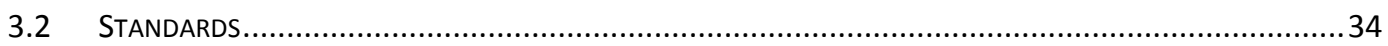

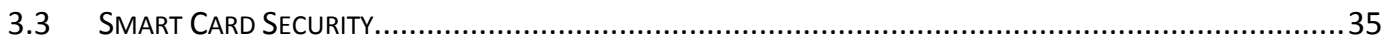

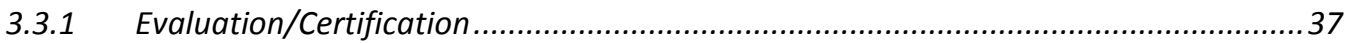




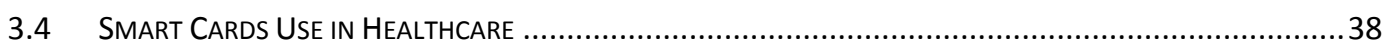

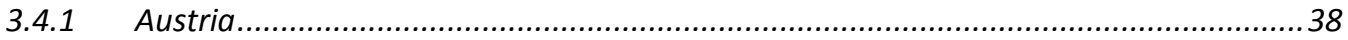

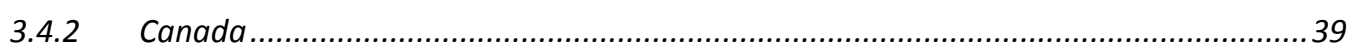

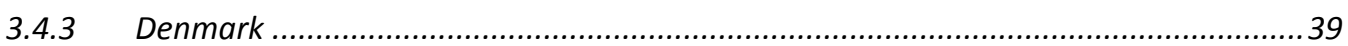

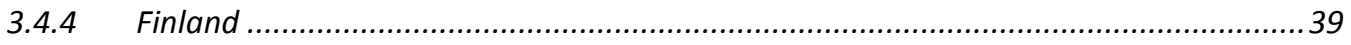

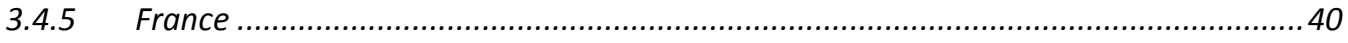

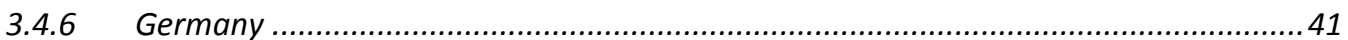

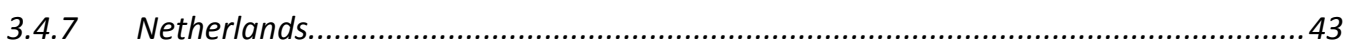

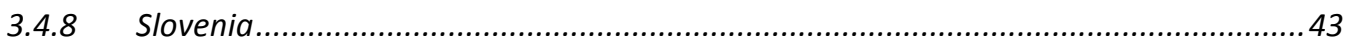

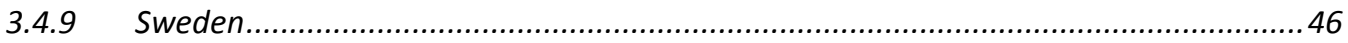

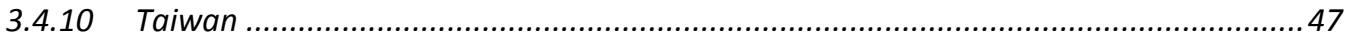

S.5 SUMMARY

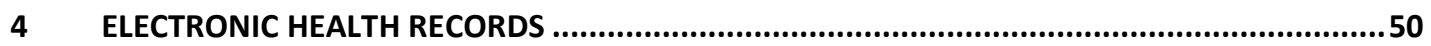

4.1 The IMPORTANCE Of EleCtronic Health Records .........................................................5

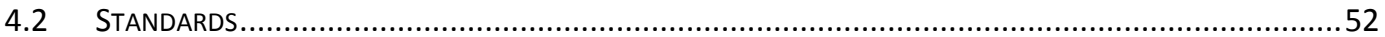

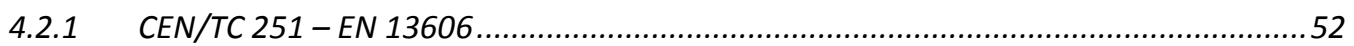

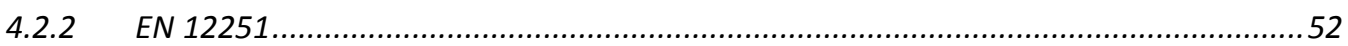

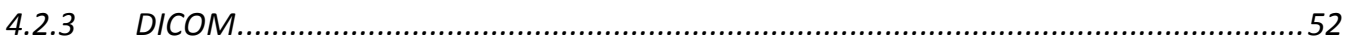

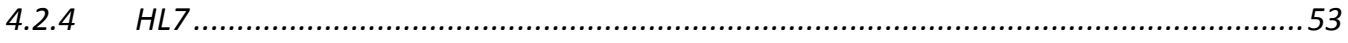

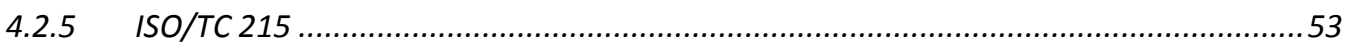

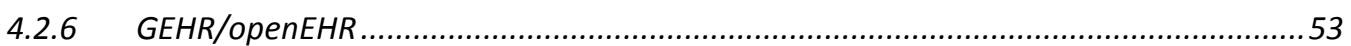

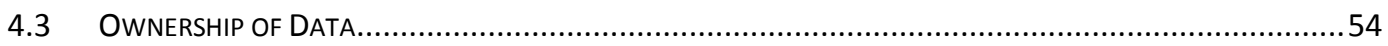

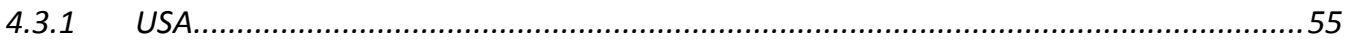

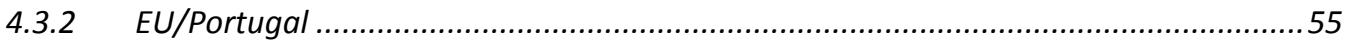

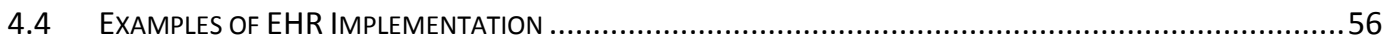

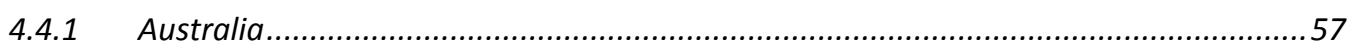

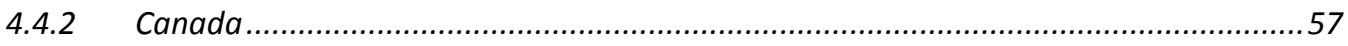

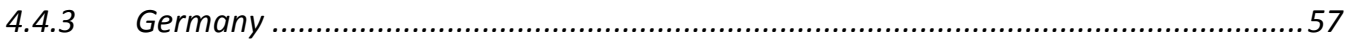

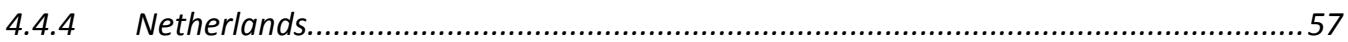

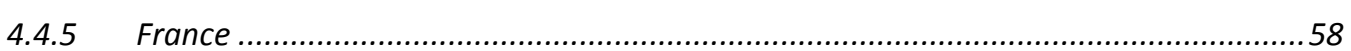

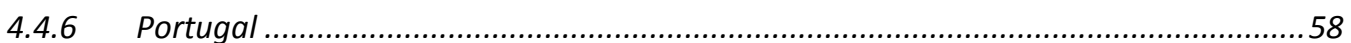

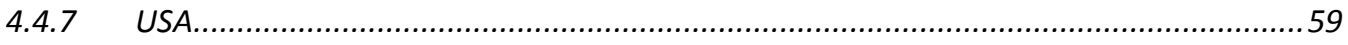

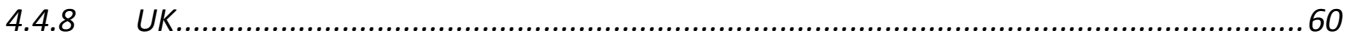

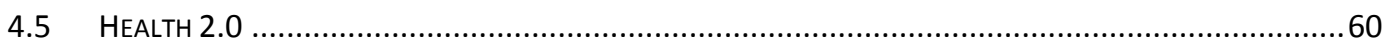

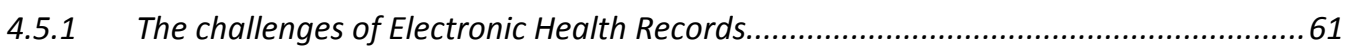

4.5.2 The concept of Personal Health Record ...................................................................... 63

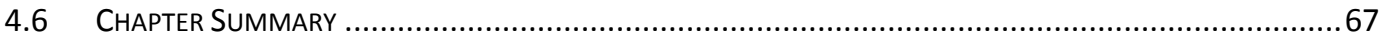


5.1 The Citizen Card Potential Role in Health Information Systems............................................75

5.1.1 Standard Healthcare Use of the Citizen Card............................................................ 75

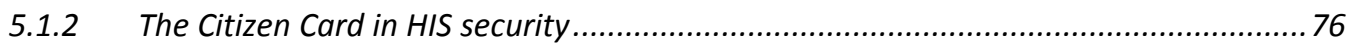

5.1.3 The Citizen Card as an Expansion Adjuvant of HIS................................................. 77

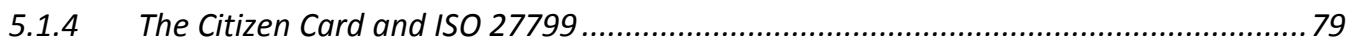

5.1.5 Anticipated Problems with the Use of CC in a HIS.................................................... 84

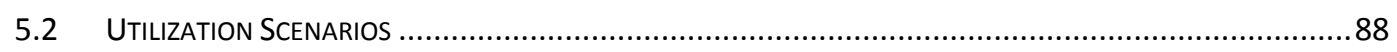

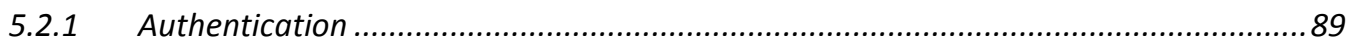

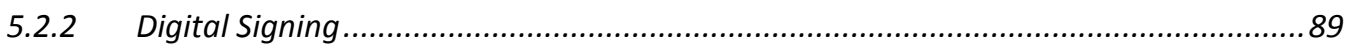

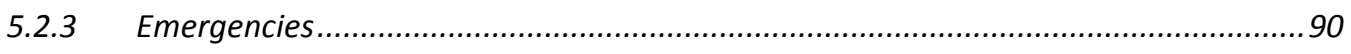

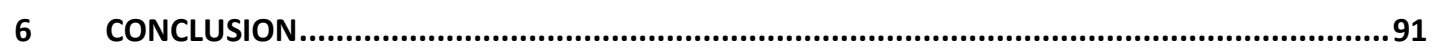

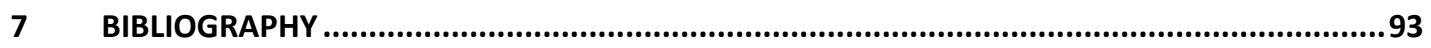




\section{List of Tables}

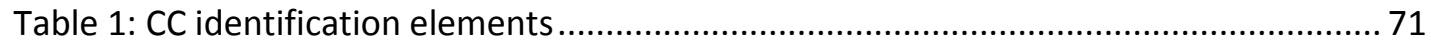

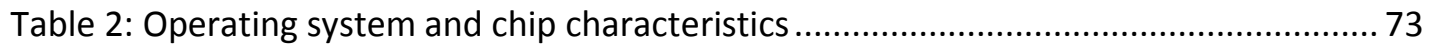

Table 3: Standards implemented by the Citizen Card PKI .................................................... 74 


\section{List of Figures}

Figure 1: Encrypting and decrypting a message ….......................................................... 10

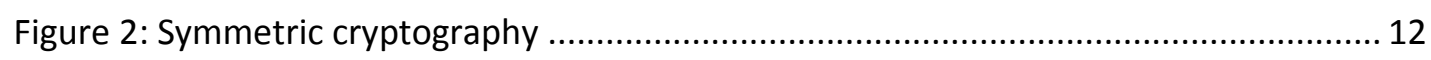

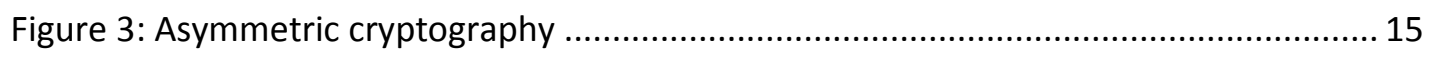

Figure 4: Confidential exchange of authenticated data over an unsecured channel............ 16

Figure 5: Summary of enrolment and use of biometric systems, as well as some of the

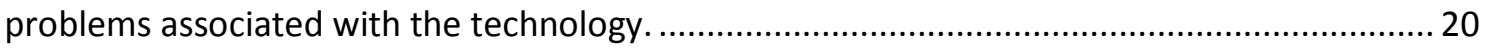

Figure 6: Cryptanalysis fields and some important attacks on smart cards ......................... 36

Figure 7: Security aspects of the CC front ……................................................................. 70

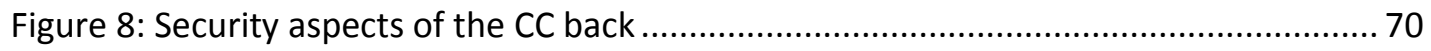




\section{List of Abbreviations}

AES - Advanced Encryption Standard

CA - Certificate Authority

CC - Citizen Card

CNPD - Comissão Nacional de Protecção de Dados (National Data Protection Commitee)

CPS - Carte Professionnel de Santé (Health Professional Card)

DAC - Discretionary Access Control

DES - Data Encryption Standard

DoS - Denial of Service

DMP - Dossier Médical Personnel (Personal Health Record)

EAL - Evaluation Assurance Level

ECC - Elliptic Curve Cryptography

EEPROM - Electronically Erasable Programmable Read-Only Memory

ER - Emergency Room

EU - European Union

e-Health - Electronic healthcare

EHR - Electronic Health Record

FAR - False Accept Rate

FEAL - Fast data Encipherment Algorithm

FRR - False Reject Rate

GP - General Practitioners 
HIPAA - Health Insurance Portability and Accountability Act

HIS - Health Information System

HTTP - Hypertext Transfer Protocol

HTTPS - Hypertext Transfer Protocol Secure

ICAO - International Civil Aviation Organization

ID - Identification Document

IGIF - Instituto de Gestão Informática e Financeira da Saúde (Institute for Health Informatics and Financial Management)

IT - Information Technology

$\mathbf{k b}-$ Kylo Bit

kB - Kylo Byte

LAN - Local Area Network

IEC - International Electrotechnical Commission

IP - Internet Protocol

IPv4 - Internet Protocol version 4

IPv6 - Internet Protocol version 6

ISO - International Organization for Standardization

ITU-T - Telecommunication Standardization Sector

MAC - Message Authentication Codes

MACC - Mandatory Access Control

NFC - Near Field Communication

NHI - National Health Insurance

NHS - National Health Service

NIST - National Institute for Standards and Technology

OS - Operating system 
PHR - Personal Health Record

PIN - Personal Identification Number

PK - Public Key

PKI - Public Key Infrastructure

RIS - Rede de Informação em Saúde (Health Information Network)

RBAC - Role Based Access Control

RF - Radio frequency

RNU - Registo Nacional do Utente (National healthcare User Database)

RTS - Rede telemática da Saúde (Health Telematic Network)

SAM - Sistema de Apoio ao Médico (Doctor Support System)

SAPE - Sistema de Apoio à Prática de Enfermagem (Support System for Nursing Paractice)

SSL - Secure Sockets Layer

SNS - Serviço Nacional de Saúde (National Health Service)

TCP - Transmission Control Protocol

TLS - Transport Layer Security

USA - United States of America

USB - Universal Serial Bus

VHA - Veterans Health Administration

VM - Virtual Machine

VPN - Virtual Private Network

W3C - World Wide Web Consortium

WPA - Wi-fi Protected Access

WEP - Wired Equivalent Privacy

XML - eXtensibile Markup Language 


\section{Introduction}

In 1984, Peter Reichertz [1] gave a lecture on the past, present and future of hospital information systems. He had large experience with those systems and pointed the trends for future development. Twenty years later, Reinhold Haux [2] revisited the subject, noting the still valid contents of the original text:

- The increase of information to store

- The shift from paper to electronic documents, and from local databases to distributed ones

- The inclusion of patients as active system users

- The use of data collected for investigation and healthcare planning and aiding, besides administrative purposes

- The shift from mainly alphanumerical data to inclusion of images, data on the molecular level and other forms of multimedia

- The multitude of new technologies used (sensors, etc), and the inevitability of ubiquitous computing

These trends for HIS (Health Information System) development are indeed the result of a continuous work in progress, especially in Portugal, judging by the immaturity of systems and the still generalized use of paper as the primary Health Record. Nowadays, the challenges of modern healthcare demand for better ways to handle the large volume of information and the level of privacy required, rightfully, by whom that information concerns - the patient. There are better ways to improve the handling and use of medical data, so the change from piles of paper to bits in computer systems is mandatory.

These changes are nowadays widespread and many hospitals in the developed world have had, for many years, computerized information systems. Most systems comprise some kind of computer based HIS, or, at least, bureaucratic information, like identification information for billing purposes. Some Investigators [3] have shown good performance from the transition to Electronic Health Records. 
The adoption of Information Technology in healthcare is generally viewed as one of the means to improve the quality, safety and efficiency of healthcare systems, although new dangers can, and do, arise from the use of technologies that expand possibilities of creation, access and use of medical data. There are, however, various ways of making the access to this data more secure, and in some cases even more secure than current paper based records. This involves:

- The computer security (machines): to prevent abuse from malicious users, with measures taken to ensure that the integrity of its software is not compromised and it is difficult to install malicious code, as well as using it without flagging alerts to system administrators. Some examples are antimalware software (centrally monitored), restricted user privileges, etc.

- The security of the servers: with the aforementioned measures as well as strict physical security (restricted access), redundancy of systems, hardware and power, with auto recover features (for data availability).

- The security of the network and communications: with dedicated networks and/or adequately encrypted communications, prudent use of wireless systems with adequate protection measures and controlled signal range, logging of critical actions, intrusion detection systems and other means of network activity monitoring.

- The physical security: Depending on the location of the hardware, additional measures may have to be put in place, e.g. in a restricted entrance room that is permanently occupied by hospital staff, these security measures may be different from those in place at a corridor accessible to anyone.

- User related security: Appropriate identity and authentication methods must be put in place to ensure that an unauthorized user does not have access to a legitimate account to access the system, jeopardizing all the measures discussed above. This means that it is pointless to design an intrinsically secure system if there are no adequate measures to ensure that an attacker could not simply bypass it by impersonating a legitimate user.

These are not, however, easy tasks to accomplish, and the transition to a fully computer based HIS has been impaired by further concerns of privacy and data confidentiality. In this respect there are repeated examples of failures and breaches of security $[4,5]$. It is best to keep in mind the dangers that come with this change of paradigm, that are recurrent in IT. 
HIS are difficult to protect from unauthorized use, because of the large and diverse number of people generally involved in healthcare providing, the unawareness and lack of interest of users about security issues and the inherent complexity of secure architectures for HIS. It has been known for a long time that login/password authentication mechanisms do not work very well in practice to secure Information Systems [6]. Users tend to be the weakest link, since they usually choose small and easy to remember password, use the same password in various applications, with many passwords being shared between unauthorized people or stored in unsafe locations. Furthermore, new steps in electronic healthcare (e-Health) for a broader, yet safer, healthcare network, require changes in HIS authentication mechanisms for the purpose of secure remote transactions of data, liability and non-repudiation. More secure authentication mechanisms are, ultimately, one of the key elements of success in the implementation a secure IT (information Technology) system [7]. Although it is of paramount importance to IT systems security, these have, too often, far from ideal authentication methods [8]. A good authentication mechanism must address and protect all discussed aspects related to the end users.

End users are often unaware of IT security concerns. HIS users are mostly doctors, nurses, healthcare technicians and administrative personnel, and these professionals often lack proper training and formation on IT utilization. HIS security suffers from this scarce knowledge of risks and their solutions.

It is impossible to write about secure digital authentication without referring, implicitly or explicitly, to cryptography, as a science which provides the means to communicate securely, hiding the contents and assuring the integrity of a message, as well as identifying the origin of a communication or message through various mechanisms, often transparently to the end user. Modern IT security relies on cryptographic methods to securely transmit, authenticate and protect data. Interestingly enough, cryptography was of great importance well before computers were a reality. There are some historical examples, like the Caesar cipher, a simple substitution cipher, used by Julius Caesar to communicate with his troops over 2000 years ago [9]. In fact, efforts to conceal a message or its meaning exist since men have learned how to write.

Traditionally, there are 3 types of secrecy systems:

- Steganography, the science behind the true concealment of the message, such as by invisible ink, hidden messages in innocent text or even in a misleading 
cryptogram, thus making secret the very existence of the message. This is often referred as security through obscurity

- Privacy systems that require special equipment to recover messages (such as speech inversion)

- Cryptography, sometimes referred as a "true" secrecy system, that relies in the impossibility (or reasonable impracticality) to understand (decrypt) a message (cryptogram), although one is aware of it [10]

These systems, individually or combined, can provide various levels of security to a message or communication, and further contribute to information security, and its three main characteristics: Confidentiality, Integrity, Availability.

The concept of remote authentication with a card has been introduced many years ago, namely by the first plastic cards by Diners Club half a century ago. Smart cards, which are chip based cards, more advanced and secure than magnetic strip ones, and allow for the use of secure authentication methods.

Smart cards are, in essence, small computers, and thus can effortlessly use strong cryptography and manage complex methods of authentication. They are often used for the purpose of securing information systems as a substitution or complement of login/password based authentication scheme and are a well documented, versatile and secure technology, very widespread. There are however several difficulties related to the implementation, integration and deployment of these technologies within healthcare in the context of a large hospital, a whole region or even a country.

Portugal is in the process of rolling out a new Identification Document (ID) for its citizens, which will aggregate several different officially issued documents. It is now available throughout the country. The Citizen Card (CC) is a smart card with strong remote authentication and digital signing capabilities by means of asymmetric cryptography. Because all the complexity involved with testing and deploying the card, as well as having the Public Key Infrastructure (PKI) in place, lead us to analyze and explore in this document the feasibility of using this the CC in a HIS setting, thus providing the basis for strong security and privacy, as well as its ease of use in Healthcare.

We will review the background for the problems and solutions presented, address plausible deployment scenarios, and explore the main characteristics of the $\mathrm{CC}$, with the intent of assessing its security, usability and usefulness, as a means of authenticating patients and 
health professionals in a HIS setting, ensuring the confidentiality, integrity and non-repudiation of medical data. 


\section{Security in Health Information Systems}

\section{The problems and solutions}

It is believed that the Hippocratic Oath appeared in the $4^{\text {th }}$ century B.C. Although there is no historical certainty about its author, it is often attributed to Hippocrates or one of its students. It has since been rewritten, to suit the values of cultures influenced by Greek medicine [11]. It mainly exposes the broad ethical standards that a sworn physician shall uphold, and, although not mandatory, it is still traditionally used by many medical schools. From the many ethical obligations in the document, from which "(...) I will do no harm (...)" is probably the best known, stands the oath for secrecy:

"Whatever I see or hear in the lives of my patients, whether in connection with my professional practice or not, which ought not to be spoken of outside, I will keep secret, as considering all such things to be private." [11].

Most professional associations and general guidelines devote great importance to doctorpatient confidentiality [12]. This is truly important for the sake of effectiveness in that relation, as well as the safeguard of patients own rights, both ethical and legal. Doctors have earned that trust over the centuries, but in the current emergent paradigm of medical data - the transition to Information Systems, some problems are posed, namely:

- The doctor is required to write down/type the relevant information in a file which he does not fully control

- It no longer depends on one person to keep Information safe

- There are other professionals which may have access to the information (nurses, health technicians, clerks, orderlies, IT personnel)

- There is the problem of intrinsic HIS security

- The potential for harm from security breaches increases with the easy transport of data and simultaneous access to multiple records from one source

Security and protection of patient health data, besides being demanded by patients themselves, is, in most developed countries, also required by law. In the United States, the Health Insurance Portability and Accountability Act (HIPAA) $[13,14]$ emphasizes, since 1996, the privacy of health information, and the need for its confidentiality, integrity and availability. The European Union has also issued guidelines on patient data protection [15], and Portugal protects health information as an individual's property, subject to confidentiality [16]. 
Handling health information is a great responsibility, and a very delicate process. HIS must be able to deal with thousands of individual records, and protect them from misuse. This is of utmost importance, since patient health data is among the most sensitive of all personal data.

The effects of aggregating data into large databases are that what could be easily seen by a dozen people about one hundred people (in a small office) may be seen by one hundred people about one million (in a region). Opportunity for wrongdoing increases the more people are involved. Some examples of misuse of large databases have been known for many years [17]. Some examples are presented here:

- As banks evolve in IT structure, almost any teller in any dependency has access to account information on any bank client. Therefore it is easier to find someone interest in accepting a bribe to look for specific clients information. This may be applicable to healthcare.

- The misuse of health data can be disastrous, such as the example of a banker with a state health commission who had access to all state patients with cancer, calling its clients loans [17].

- Prescription systems allow for identification of trends in individuals, which may then be used to direct specific unsolicited products or services. As all patients are identifiable when they fill a prescription, the resultant database could endanger those people privacy.

- Companies holding large databases, while keeping the data private, may be bought by larger companies with interest in those databases, such as a drug company buying a health systems company.

- Many companies in the USA use medical records in their hiring decisions [17].

Besides security threats concerning the data privacy, HIS brings other problems on integrity and availability of data, such as hardware/software bugs, systems failure and communication problems (messages unavailable or misinterpreted).

In 2004, the Portuguese National Committee on Data protection (Comissão Nacional de Protecção de Dados - CNPD) audited 38 hospitals on personal data processing and protection [18]. Among other problems, they found fourteen applications without password, passwords visible on stickers next to workstations on two hospitals, as well as several users stating that the passwords were, literally, their own name. This highly negative state of affairs was no surprise, as previous works on the subject had already been raising similar concerns for the last 
30 years [6]. Generally, studies concerning the security of EHR have shown inadequate practices, urgently requiring improvement [4].

The intrinsic value of the internet, with its promising information ubiquity, along with the possibility free and practical data communication between distinct institutions, is also its main danger, lest that information should fall into the wrong hands. Using the internet for crossinstitutional communication also dramatically increases the danger of sensitive patient health information being manipulated or abused, by falling into the wrong hands.

As defined by the European Union (EU) [15]:

- Data protection aims to protect the fundamental rights and freedoms of natural persons and in particular their right to privacy with respect to the processing of personal data.

- Data security aims to protect personal data against accidental or unlawful destruction or accidental loss, alteration, unauthorized disclosure or access, in particular where the processing involves the transmission of data over a network, and against all other unlawful forms of processing.

This is traditionally tackled by upholding three main security characteristics[19]:

- Confidentiality: assurance that patient data is not made available or disclosed to unauthorized individuals. This is a serious matter, as the aggregation of information brings about new threats, with the easier access: the likelihood that information will be improperly disclosed depends on two things: its value, and the number of people who have access to it [17].

- Integrity: ensures that patient data cannot be changed or deleted by unauthorized individuals or parties. Software bugs or hardware failures can also cause data loss or wrong data.

- Availability: upon demand patient data can always be accessed and used by authorized people.

In HIS it is also very important to be able to guarantee:

- Authentication: the corroboration that a person is the one claimed.

- Non repudiation: Someone cannot reasonably deny certain actions.

- Accountability: the actions of a person, especially the modifications that he/she performs on data stored in an EPR, can be traced. 
The EU commission also issued the Community framework for electronic signatures [20], which regulates secure data exchange, and created a foundation for the legal equivalence of electronically signed documents and its hand-signed counterparts, although only through the use of qualified and accredited electronic signatures, which guarantee such equivalence.

To understand how we can start to integrate and implement all these security concepts and properties into a HIS one must first explore what the science of Cryptography has to offer in terms of secure algorithms and communication protocols.

\subsection{Cryptography}

When we post a written letter, we will probably use a sealed envelope, which has the primary purpose of keeping its contents hidden to prying eyes. We generally consider the contents of personal correspondence to be private, and that the practice of sealing the envelope effectively protects its contents, even if they are not sensitive. Nowadays, it is certainly more private to use the regular mail than email, because the email systems do not protect the contents of the messages, and while they travel through multiple servers and nodes throughout the internet, until they reaches their destiny. Email can be screened and copied at any of these locations, and messages are also kept in servers, where they can be read by anyone with access to the storage.

It is, however, possible to secure email, e.g. by securing the message while in transit or protecting its contents. Both approaches generally make heavy use of cryptographic algorithms.

Cryptography is a science with high historical influence for the past 2000 years. It has been used by lovers, the government and the military, with the main purpose of withholding information from unintended recipients [9]. From a science known and practiced by few, it grew to becoming a well established academic discipline, and pervasive in modern digital societies. Contrary to items stored in a vault, encrypted messages are visible, but have their meaning hidden and therefore of no use to the eavesdroppers unless he is in possession of the means to decrypt it. Anyone may see the message, although only people in possession of the cipher key may read it. This is illustrated in Figure 1, and consists of "disguising" an intelligible message in a code, by applying a set of rules and an encryption key (encryption algorithm), with no use for any person without the means to revert the process (possession of the decryption key and decryption algorithm or process). 


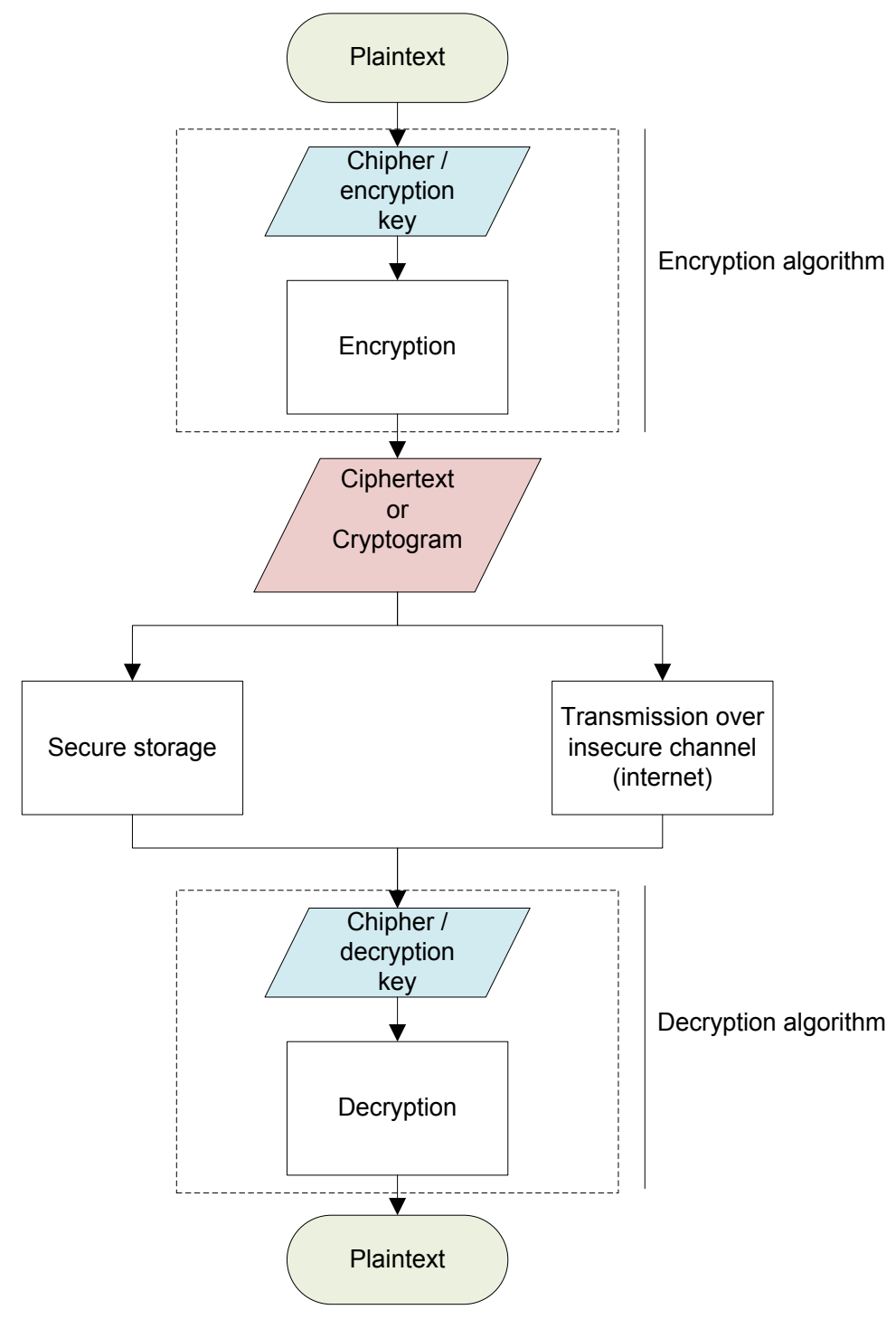

Figure 1: Encrypting and decrypting a message

To make effective use of cryptography one must be aware that:

- As the keys must remain secret, there's the issue of securely distributing the keys to the intended people (senders and recipients). A key compromise will thwart security: communicating the key by an unsecure channel (email, telephone, etc.), storing the key in an accessible location (computer, post-it, etc). This means the key must be well guarded. It is pointless to secure a valuable message with a strong algorithm if the key can be easily discovered or otherwise obtained. Keys based on own names, known dates, sequences of numbers ("1234") and other common keys and passwords ("password"; "password1", etc) are widely used and are automatically employed by many hacking tools.

- The Encryption Algorithm, or Cipher, as well as the keys, must be of adequate security and strength, that is, the algorithm/key must reasonably withstand expected attacks. 
Steganography, or the science that studies dissimulation of messages, aids to further protect a given message, by effectively dissimulating an important and confidential encrypted message in a non sensitive, easily readable and inoffensive text or image. This adds another layer of security, comparable to having a strong vault disguised in a plain looking wall: even if a specialized burglar could know how to open it, he would have to discover its existence and location.

Most of the methods used to guarantee the data security and data protection of patient health data are based on cryptographic procedures. By encoding messages written in plain text, which everyone could read, in a cipher text, which can then only be read by authorized persons, information can be securely transferred. The algorithm, which uses a person-specific parameter, the key, describes the way encryption is performed [21].

There are a wide number of algorithms, classical and contemporaneous, that can be used to encrypt messages. They vary from the naive Caesar's substitution cipher to mathematically challenging processes, such as those used by RSA algorithms. Only some, with special interest to HIS security will be presented.

There are mainly two types of encryption, symmetric and asymmetric. This is a fundamental distinction based on the keys used to encrypt and decrypt the cipher text (or cryptogram). When the same key is used for both processes depicted in Figure 1, that is, the same key will encrypt a message and similarly revert it to its original format, the encryption system it is called symmetric. When the key used to encrypt cannot be used for decryption and vice versa (and thus the whole process depicted in Figure 1 cannot be completed without the two keys), the encryption system is asymmetric. This also has profound implications in the possible uses of encryption schemes, as we will see.

\subsubsection{Symmetric cryptography}

Symmetric cryptography has the advantage of being quite easy to implement and very quick to encode or decode. The same key is used in both processes. This is the classic cipher.

Symmetric encryption has the disadvantage of distribution of keys, since this cannot be done over an unsecure connection (see Figure 2). Secure key exchange is difficult, because the creation of a secure communication channel to transmit a key would depend itself on another key, so that the transmission of the keys has to be made in advance, over other channels (e.g. secure courier). There is cost and delay associated with this paradigm [22]. 


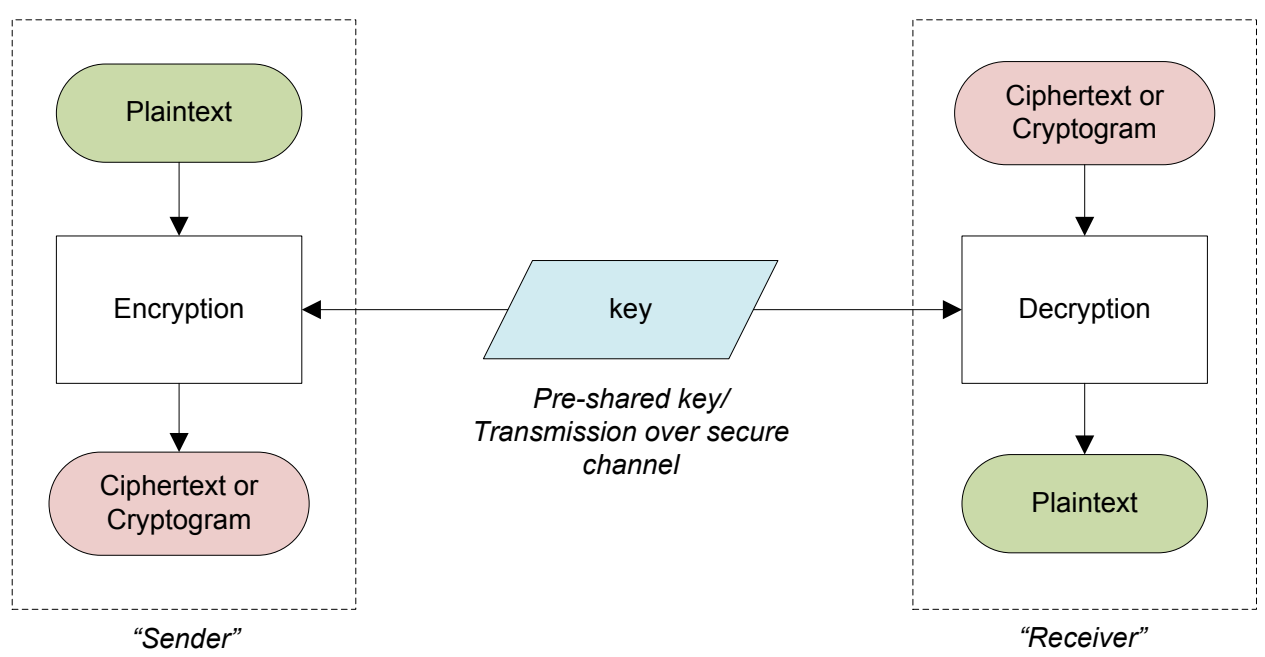

\section{Figure 2: Symmetric cryptography}

Some types of symmetric encryption algorithms and related processes, as well as some advantages and disadvantages, are presented.

\section{Block Ciphers}

Block ciphers organize fixed-length groups of bits, which are termed blocks. We take number of bits of plaintext, say, 128bit, and transform it into a ciphertext of equal length, by applying the encryption algorithm. The key, along with the algorithm chosen, determines the exact transformation.

The Advanced Encryption Standard (AES) algorithm, with a block of length of 128 bits, supporting key lengths of 128, 192 and 256 bits, was chosen by NIST from an open competition in October 2000. It is based in the Rijndael algorithm (designed by the Belgian cryptographers Vincent Rijmen and Joan Daemen). Former standard Data Encryption Standard (DES), developed at IBM and published as a standard in 1977, and triple-DES, with single, double or triple keys, are still used, and the later, with more than one key, is expect to resist attacks for a few years[23].

\section{Stream Ciphers}

Instead of being applied to blocks of data, these algorithms typically are applied to bits. Stream ciphers can be designed to be extremely fast in the process of encrypting/decrypting. It is the process of combining a variable bit length key to the plaintext, usually by the use of a XOR operation (exclusive OR, bit by bit), producing the ciphertext. A synchronous stream 
cipher denotes a keystream that is generated independently form the plaintext/ciphertext. A self-synchronizing keystream is dependent on the plaintext/ciphertext [24].

The one-time pad is a randomly generated key used only once (hence one-time) to encrypt a plaintext, with the key length (in bits) as large as the plaintext itself. This leads to a level of security untouched to other techniques, and renders it theoretically unbreakable. This perfect secrecy is thwarted by the extreme difficulty in distributing very large one-time keys. It calls for difficult solutions such as codebooks, known by both parties, filled with very large disposable keystreams, distributed beforehand in person, for instance. Although they have been used during wartime or extremely secure diplomatic channels, they are unpractical [25].

Nowadays, there is no de facto standard in stream ciphers, but the most widely used is RC4. Certain modes of operation of block ciphers, e.g. using them to generate keystreams, render them as stream ciphers.

\section{Other applications of symmetric cryptography}

\section{Hash functions}

Hash functions are one way algorithms that return a bit string, or hash value, based on an arbitrary block of data. An ideal hash function creates a value that cannot be used to find the original message to which it was applied, is unique to that particular message and makes infeasible to change the message maintaining the same result of the function (the hash value). Because of these characteristics, these hash values are also referred as digital fingerprints, or checksums. Their use is variable, but some of the main uses are to verify integrity of data, detect duplicates and uniquely identify files.

Merkle-Damgård or MD strengthening of a hashing function, the most recent being MD5 (1991) is a 128bit hashing function quite susceptible to attacks [23]. Collisions (finding another set of data with the same hashing result) were found in 2004. SHA-1 is also bound to have collisions found, because search algorithms were presented, and recognized by the National Institute of Standards and Technology (NIST), that recommends its substitution [26]. These functions are not recommended for signature purposes. SHA-256, SHA-224, SHA-384 and SHA512 are candidates for substitution, but NIST will be holding an open competition for the new hashing standard. 


\section{Message Authentication Codes (MAC)}

Their use is mainly to authenticate messages. MAC's are generally based in hashing and block cipher functions, but are faster. They are widely used in smart cards. As the MAC is created using a secret key applied (with a hash function) to the message, a message received with a corresponding MAC is probably both authentic and complete.

\subsubsection{Asymmetric Cryptography}

Whitfield Diffie and Martin Hellman, with their 1976 paper "New directions in cryptography" [22] revolutionized the field by introducing new methods of key distribution, which gave origin to the so-called Diffie-Hellman key exchange. Asymmetric Cryptography was born with these investigations, answering two fundamental problems faced with symmetric cryptography [27]:

- Key distribution is difficult, especially for communication between people that have never met, and thus have to agree on a key beforehand.

- The problem of signatures in digital documents, or how to demonstrate the origin and validity (thus non-repudiation) of purely digital documents, that may otherwise be easily forged.

These apparently unsolvable problems are actually related, and can be solved by the same process. Since 1950/60 two novel methods of authentication were known. The first, used by the military, served to identify friends and enemies. It was a cryptographic challenge-response paradigm that allowed to identify friends by the correct response, and was immune to eavesdrop because messages were unrepeated, as a new random challenge was used every time it was used. This method is best known by the keyword challenge and response often depicted in movies by military personnel, but was also used at radar stations, to identify planes. It had the disadvantage that anyone taking control of the cryptographic keys in one of the challenge locations could provide the correct answers to any other location. A second authentication process (or an improvement to the existing paradigm) was developed to overcome some login/password vulnerabilities, because password tables stored in the servers (match tables to keywords entered in order to authenticate users) could be seized and all logins compromised. The use of a one-way function, a hash function, which is easy to compute, but very difficult to invert, allowed storing the password products of the function for authentication, making the password table of little use to intruders [27]. 
Public key (PK) cryptography can be seen as a one way function with a backdoor which is known only to one person/entity. If a challenger poses a question to which it doesn't have an answer, but still can verify the correctness of that answer, the problem of key distribution could be partially solved. Someone can encrypt a message with the one way function, knowing that only the one with the information of how to invert the function could retrieve the message. The one way function could be made freely available, and thus the name Public Key Cryptography (see Figure 3).

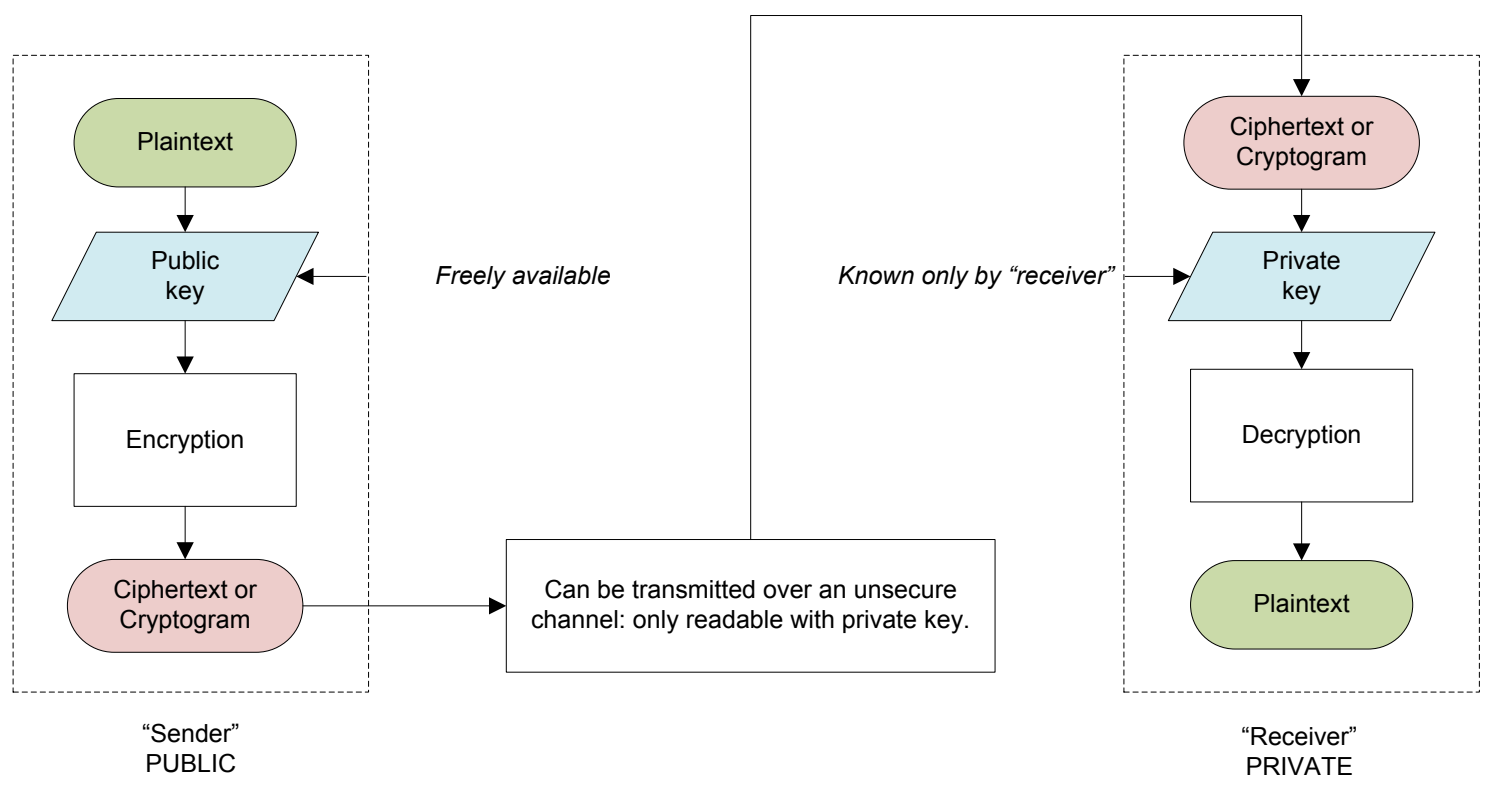

Figure 3: Asymmetric cryptography

In asymmetric algorithms, two different but mathematically corresponding keys are used for encoding and decoding. Although the keys are paired, they cannot be derived from each other. A pair is composed of a private and a public key. The private key is secret, but the public one is publicly available. The only way to decode a message created with one of the keys is by using the other. This allows for easier key distribution, as the public key may be in a public repository. It also assures that a message decoded by the public key was indeed created by the private key (and, thus, its owner), while a message encrypted with the public key can only be read by the owner of the private key. This effectively allows that one may authenticate and encrypt a message, only readable to the recipient, by combining the public key of the recipient and the private key of the sender (see Figure 4). Still, there is the problem of trusting the keys available, which is why there is the need for certificates (discussed later in the text) issued by authorities in which we choose to trust, that extend their trust into other entities. For instance, the ID 
Asymmetric cryptography is, nevertheless, a much slower (and computationally intensive) method of encryption compared to symmetric cryptography. So it has been proposed that this process could be used to share a common secret, a key subsequently used in a symmetric algorithm. The concept behind this key exchange was first described by Diffie and Hellman [22], hence the Diffie-Hellman key exchange. One of the variations of this concept is depicted in Figure 4.

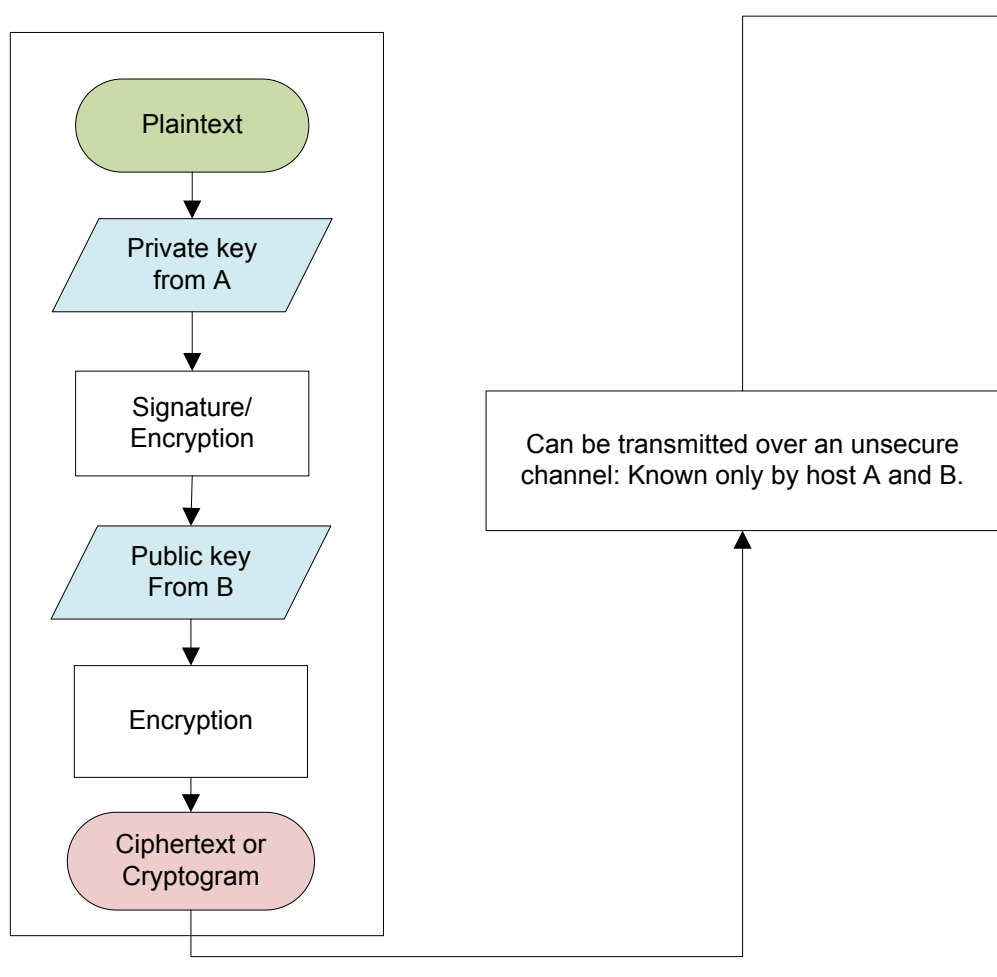

Host A

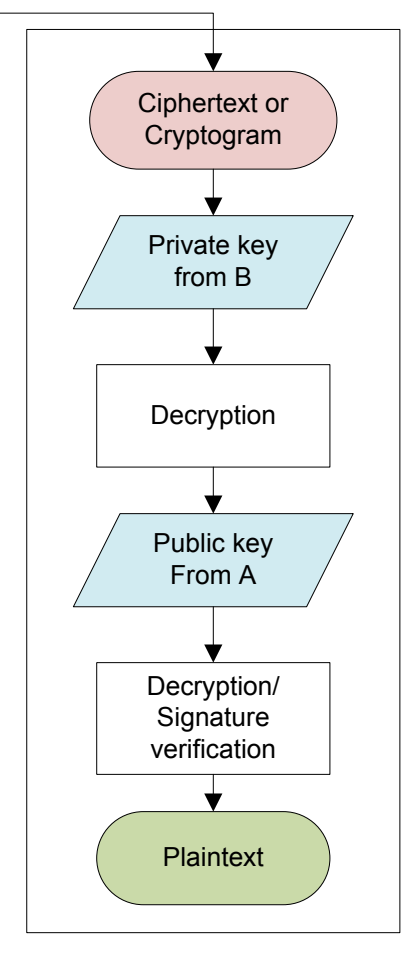

Host B

Figure 4: Confidential exchange of authenticated data over an unsecured channel

\section{The RSA algorithm}

The RSA is a public key algorithm invented by Rivest, Shamir and Adleman in 1978. It is the most widely adopted public key algorithm, having lost its patent in 2000 . There are still no known methods of breaking RSA efficiently, although the solution is theoretically simpler than factoring a given large number $\mathrm{N}$, the product of two large primes $\mathrm{p}^{*} \mathrm{q}-$ which is the basis of RSA encryption. The time needed to factor such a large integer, opposed to the relative simplicity of finding large primes to the composition of the modulus, leads to relative ease in implementing a secure encryption. The current record for factoring a large number is 200 digits (663 bits), known as RSA-200, was factored in May 2005, and was part of a contest - the 
RSA Factoring Challenge - which ended in 2007 [28].The minimum recommended key length in 2006 was 1024 bits (309 digits - $2^{80}$ steps in factoring such a number). Given today's and future computational power, a modulus of 1536-2048 bits (463-617 digits) is recommended for 10-15 years security [23].

Despite 20 years of experience with RSA, and many papers written about it, it is still hard to implement this algorithm without flaws, making its deployment theoretically vulnerable to various attacks. Although it has not been compromised, standard PKCS\#1v1.5, for digital signatures, for example, is not ideal, and various exploits have been delineated [23].

Currently the cryptographic community believes that one of the best ways of using RSA is the RSA-KEM mode: this is a so-called hybrid mode in which RSA is only used to transfer a symmetric session key (see figure above), while the plaintext is encrypted and authenticated using a symmetric algorithm with this key. This allows for less computation overhead, while maintaining secure key exchange and secure communication. This is the way most secure internet sites communicate with our computers (see 2.3.3 HTTPS).

\section{Elliptic Curve}

An alternative to RSA is the Elliptic Curve Cryptography (ECC), created in 1985 and coinvented by V. Miller and N. Koblitz. ECC is gaining momentum, and has been officially adopted by the governments of Germany, USA and Austria. It is more complex than RSA, but has shorter key lengths for similar security. It also has some disadvantages over RSA, such as being slower at signing documents [29].Its public key scheme is currently one of the most secure per bit. This means that it possesses highly wanted properties like less processing power, storage, bandwidth and power consumption. ECC has become the standard to protect U.S. government mission critical information and also sensitive and unclassified data. In their set of recommended algorithms, known as "Suite B", the Elliptic Curve Digital Signature Algorithm was chosen for authentication [30].

\subsection{Authentication}

HIS can take advantage of advanced cryptography to secure data, however the practical methods require a detour for a brief introduction to authentication schemes used nowadays. To secure information systems first there is the need to define what can be accessed, by whom, when, from where, and in what circumstances, thus defining user roles and permissions. The aim is to ensure who is who, and what can he do after being after being authenticated. This is of vital importance [31]. 
A user can authenticate himself by three classic methods: by providing something he knows (e.g. a password); something he has (e.g. a smart card or other token); or something he is (e.g. biometrics - gait, fingerprint, etc.). A combination of these methods can be used to enhance security [8].

\subsubsection{Passwords}

Passwords need to be difficult to guess, which is in direct conflict with ease of use. Humans tend to forget non meaningful strings and thus have a tendency to pick non random passwords that are very easy to remember. These easy passwords get less secure each time they are used, because they are easier to spot by eavesdroppers. On the other hand, administration and deployment of passwords based systems is widely used, easy and cheap, hence its broad use [7].

Most hospitals in Portugal use passwords for authentication in the majority of applications installed. The consequences have been reported by Portuguese Data Protection Agency [18]. They have studied data protection methods in hospital computerized systems, and found several computers with login passwords written in post-its, users confirming sharing their passwords, unsecure passwords, such as names or the same as logins, etc.

\subsubsection{Biometrics}

These are many times referred to as the "almost perfect" solution for identification, and used many times for local authentication. The key argument is that there are built around unique, unrepeatable characteristics of the human body or behavior, which can make identification of a human irrefutable. Being based in something we are, they are irrevocable, and it is very hard to erase or lose a characteristic used in biometric identification (such as removing fingerprints from fingertips). There are however various arguments against biometric technologies [32]: They are fairly expensive to deploy; the user can not change a compromised biometric; there are various known attacks on biometric systems; there are concerns on privacy [33] and usable biometrics also have errors, especially in identification mode when confronted with very large user databases. Another aspect, often depicted in fiction work, is the security of the owner of the biometric, as perpetrators, unable to learn or steal the feature, may physically harm the user. An example is the finger mutilation of a car owner with a fingerprint security system by car thieves in Malaysia [34]. 
Some people are unable to use biometric systems, as the enrolment success is close to, but not $100 \%$. Examples are:

- Hand/palm recognition and fingerprints: people with no fingers, paralytic, with serious skin diseases (with no fingerprints or unreadable ones), or other diseases that impair the use of hands, such as arthritis.

- Voice recognition: some people cannot talk

- Iris or retina: there are diseases that affect this biometric and some people do not have eyes

- Face recognition: cultural hiding of women faces.

Biometric technologies rely on enrollment on a system, by "capture" of certain physical or behavior characteristics (biometrics). This can be made by video, photo or other method, which, after processing, produces a template for posterior comparison. As there are no known ways to effectively reproduce all the variables involved in collection and posterior presentation of such biometrics (position of finger or eye; subtle voice changes; lighting conditions; different equipment, etc), some tolerance shall be given, and a threshold for positive identification is created, otherwise the user has to repeat the process. As Biometric systems can identify a person by reading the biometric and comparing it to a database (1- $\mathrm{N}$ : identification) or to a single record (1-1: verification, e.g. after a login), different thresholds are needed, in order to reduce the number of tries needed to correct identification (false negatives or False Reject Rate - FRR), while keeping low false positives, i.e. people that successfully login identified as someone else (false Accept Rate - FAR). In identification mode the resemblance (threshold) between the trait collected and the template must be highest, or else the FAR will be unacceptable (the chance some of one's characteristics are present in another individuals template are greater the larger the database). As a result of this, the FRR will be higher (as more resemblance is required to the stored template) and the individuals will have to repeat presentation of the trait more often.

It is also possible to spoof some of the biometric systems available, by reproducing the feature of a legitimate user, which may be unaware. The main problem with this kind of attack is that we cannot hide most of the biometrics in use, as we cannot easily hide who we are. Biometric features are effectively chosen because of their ease to use, hence, to present. Examples are successful reproduction of fingerprints left in glass or fooling facial recognition with a photo [32]. 


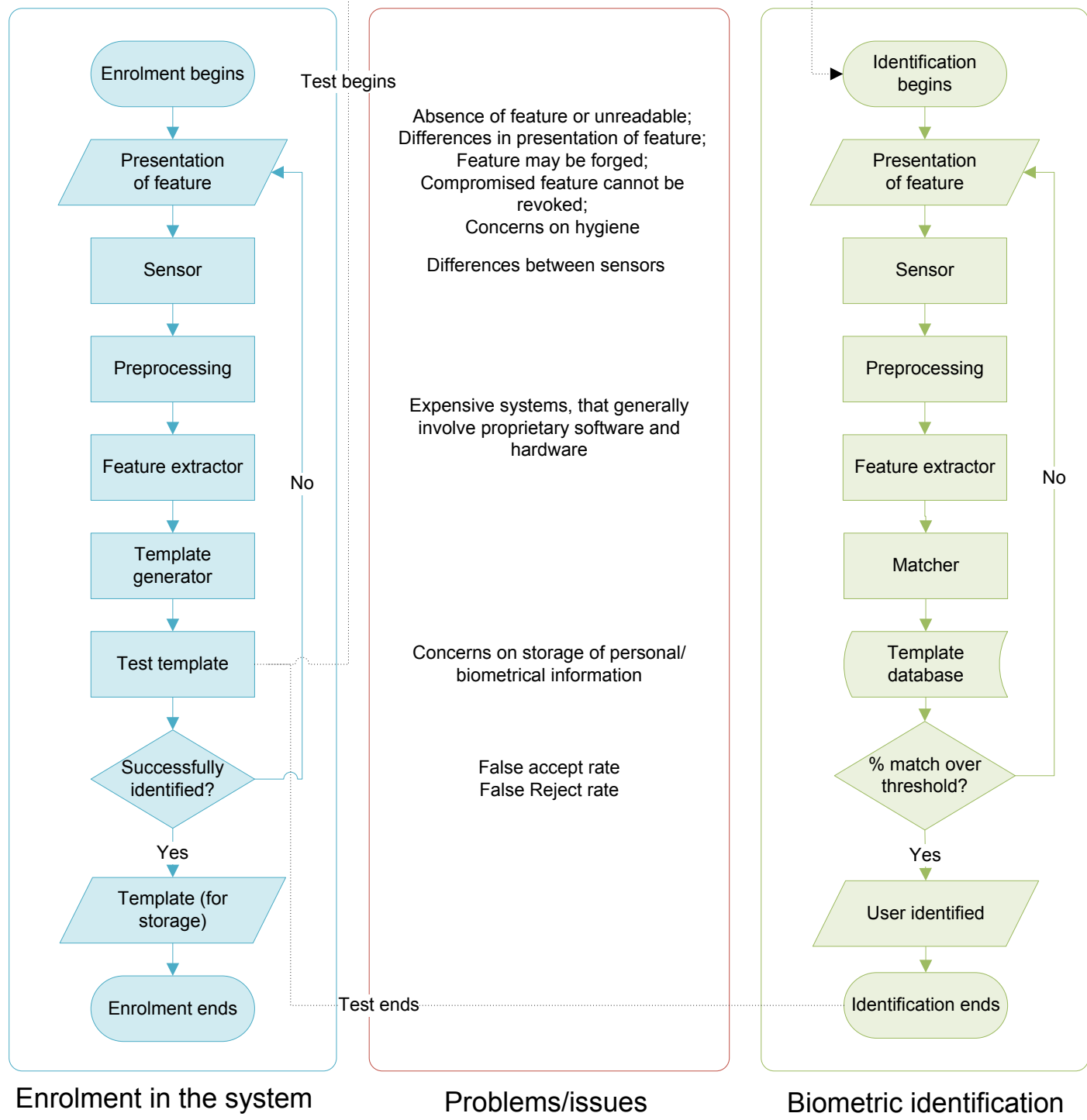

Figure 5: Summary of enrolment and use of biometric systems, as well as some of the problems associated with the technology.

An example of broad use of this technology in the Serviço Nacional de Saúde (SNS Portuguese National Health Service), is the Alert $^{\circledR}$ system [35], an emergency room (ER) oriented information system deployed in many Portuguese hospitals. This system uses, mainly, authentication by fingerprint. This same method is being introduced to control healthcare workers schedules, substituting the previous use of magnetic cards (simple token) and other systems, such as signature.

\subsubsection{Token authentication}

This method is based on the possession of something physical that can be uniquely associated with a certain entity and very hard to reproduce. It is very common to use smart cards as security tokens. They are very flexible and secure: but also have some drawbacks. If 
lost, there is the possibility of system compromise. They can be, however, revoked, and reissued. One also needs to carry it, it is not free and requires some effort to deploy and manage. If combined with a password or PIN, a token can safely store or generate multiple passwords and secret codes, which obviate the task of remembering multiple, changing passwords to one of remembering only the single password needed to access and activate the token. It also provides compromise detection, since its absence is observable (opposed to a password), and it provides added protection against denial-of-service (DoS) attacks (a person can try to login and lock multiple accounts in a login/password authentication scheme, but with the requirement of a security token, only someone with a valid active token can try to logon into the system).

\subsection{Protecting HIS}

We have already seen why these systems must be protected, what to have in mind when doing so and some of the technologies at our disposal. In this section we will discuss specific problems, as well as how to deal with HIS security in its most important aspects.

\subsubsection{Steps for a Secure HIS}

Dr. Ross Anderson, in a paper for the British Medical Association policy on Security of Clinical Information Systems [17], regarding the introduction of a nationwide NHS (National Health Service) network, presented several principles to achieve the desirable privacy, which we discuss:

1. "Each identifiable clinical record shall be marked with an access control list naming the people or groups of people who may read it and append data to it. The system shall prevent anyone not on the access control list from accessing the record in any way."

- This principle relates to Who can access and What can they do with the data. As the databases become more ubiquitous, grows the necessity for enforcing restricted access. While 20 people in a ward may access 30 records on paper, potentially thousands of people might be able to access millions of records nationwide in an EHR without proper restrictions.

- Records could remain as they are in paper, i.e., accessible to a group of people that created the information, such as a GP (General Practitioner) and be externally accessed only by explicit consent by the patient, with or without restrictions.

- This policy must be known by the patient, so that he can make decisions regarding it. 
2. "A clinician may open a record with herself and the patient on the access control list. Where a patient has been referred, she may open a record with herself, the patient and the referring clinician(s) on the access control list."

- When the patient is referred for a specific disease by his GP, there is obvious interest in that both clinicians and the patient can access the data, so the new record may have those permissions.

3. "One of the clinicians on the access control list must be marked as being responsible. Only she may alter the access control list, and she may only add other health care professionals to it."

- Other than the patient, only healthcare professionals may be able to access the EHR. There must be a responsible doctor for that document, who may be the patients GP or the hospital consultant that is in charge of treating him

- Healthcare professionals must be both responsible and liable for misuse, and the professional status has to be defined, as some professions may not be clearly classified, such as social workers.

- When a record is transmitted and/or given access, care must be taken to ensure responsibility and liability on the receiver.

4. "The responsible clinician must notify the patient of the names on his record's access control list when it is opened, of all subsequent additions, and whenever responsibility is transferred. His consent must also be obtained, except in emergency or in the case of statutory exemptions."

- The need for a patient to give explicit consent on the people that may access his record is difficultly overstated. In emergent situation, justification for the action has to be produced

- The responsible for the professional and/or the EHR and the patient could be notified in case of exceptional access and new additions to his "allowed" list.

5. "No-one shall have the ability to delete clinical information until the appropriate time period has expired."

- Besides setting legal mandatory persistence of records, this principle implies that records can be deleted, and sometimes, should, as in the case of a copy or access issued for the purpose of a single specialist consult.

- For some information, this could mean the lifetime of the patient (surgery report), whilst for other, this could mean a few years or less (normal blood workup). 
6. "All accesses to clinical records shall be marked on the record with the subject's name, as well as the date and time. An audit trail must also be kept of all deletions."

- This feature is important in many levels. It allows for tracing and punishing of misuse, fraud or unauthorized access. It may also allow (depending on implementation) for reconstruction of some deleted data.

- The HIS must be engineered with the same care and to the same standards that are expected in life support systems, as life/death decisions can equally be made from the information conveyed from it, such as a "Do not resuscitate" notice.

7. "Information derived from record A may be appended to record B if and only if B's access control list is contained in A's."

- When a patient has two records, different information can be viewed by different people. The purpose is to keep information considered sensitive. The flagging of such hidden information (explicitly, or conspicuous lack of record parts) is controversial, as the mere knowledge of its existence could lead to inference about it. France's EHR (Dossier Medical Personnel) is dealing with such controversies [36].

- The release of hidden information should be considered a special action, and logged as such.

- The system should be able to prevent accidental leakage of data (such as accidentally appending clinical records to a mailing list).

8. "There shall be effective measures to prevent the aggregation of personal health information. In particular, patients must receive special notification if any person whom it is proposed to add to their access control list already has access to personal health information on a large number of people."

- This aggregation control is of utmost importance, as even in a local level, some hospitals have records in over a million people, with all users having access to that data, with less than optimal control of the access use or misuse.

- This kind of access control (or lack of it) should preclude participation in even larger networks, as "having 2,000 staff accessing a million records is bad enough; but the prospect of 200 such hospitals connected together, giving 400,000 staff access to the hospital records of most of the population, is unacceptable." [17].

- We should keep in mind that making data anonymous is quite hard, especially if a complete medical record is at hand, as certain medical details crossed could easily result in the identification of a single person: Caucasian, married, two daughters, 35 years old, leg surgery from car accident in $2004 . .$. 
- Special techniques to prevent inference from the data (statistical security) have been used in census databases, and can also be used for the purpose of mere statistical analysis.

9. "Computer systems that handle personal health information shall have a subsystem that enforces the above principles in an effective way. Its effectiveness shall be subject to evaluation by independent experts."

- All the hardware and software used in a HIS must obey all the principles above, and even enforce them. Testing for software bugs or planning for hardware failures is part of it.

- User authentication and access control is of paramount importance, as well as communications security. These features have to be tested by independent parties.

This list is not an exhaustive one, yet it uncovers several key aspects that are often forgotten. We will now tackle some of the solutions to these problems.

\subsubsection{Public Key Infrastructure}

Following the EU directives, the need for interoperable HIS requires the existence of a trustworthy Public Key Infrastructure (PKI) based communication and cooperation platform as well as a properly deployed privilege and access control infrastructure [37].

A document can be signed by calculating an unambiguous checksum with a given hash function of the electronic file, which is afterwards encrypted with the private key of the author. Any person with access to the correspondent public key can decrypt this checksum, and compare to the result obtained by applying the same hash function to the document. If the two are the same, the document has not changed since it was signed and was in fact created by the person with the matching private key. If we know for sure that the key pair belongs to a certain individual (e.g. the public key was personally given to us) we are certain of the document origin.

Similar steps are taken with authentication, as if we know for sure who is the owner of a given public key, we can encrypt a request with our signature and then encrypt the result with the person's public key. This way we know for sure that only the intended recipient opened the request, and he now believes it came from us, as it was encrypted with our own private key (see section 2.1.2 above and Figure 4).

The problem here is how to make sure the public key belong to a certain individual: This is accomplished by means of a certificate, a piece of digitally signed data that binds the public 
key to the identification data of the key holder. A trusted third party verifies the identity and certifies the public key[38]. This is part of the PKI.

A Public Key Infrastructure is the complete infrastructure necessary to carry out procedures involving public key cryptography allowing deployment and maintenance of digital signing and authentication schemes, since key binding to revocation, as well as the means to verify the revocation status.

Key emission/binding is done by a Certificate Authority (CA), which is a third party, often a trusted company or agency (governmental or otherwise). It binds a public key with identification, by including and signing a certificate providing the identity and conditions for validity, such as expiry date. The public and private keys can then be distributed, with the private key securely exchanged with the user. It essentially publishes the public key for a given person or entity, signed by its own certificate. It creates a trust network in which this trusted entity (the state or a company), owner (creator) of an original certificate, extends its trust to others, be it other issuing entities or people. By assuring a tightly ruled network (infrastructure) the trust in certificates is as good as the trust in the primary entity.

A revocation list is also provided in the PKI context, readily available, that terminates validity on certificates. These processes may be implemented by means of a standard for PKI, X.509, coordinated by the ITU-T (Telecommunication Standardization Sector - International telecommunication Union), which addresses formats for public key certificates, certificate revocation lists, attribute certificates, and a certification path validation algorithm [39].

PKI use in Hospitals is thought to be a necessity, as the major part of medical documents in a hospital are relevant for signature and therefore will have to be electronically signed in a EHR environment. For this reason, the PKI is deemed indispensable for the further development of electronic patient records, as it provides the means to sign electronically on an irrefutable way. In clinical routine the procedure will probably only be accepted if the performance of the signature functionality and the necessary user interfaces optimally support the complex business processes $[21,38]$. That is to say that acceptance will be directly dependent with perceived benefit and ease of use.

Electronic Health Records (EHR) are part of HIS and may be deployed in several architectures, but some requirements are common. Among others, the need for information confidentiality and integrity, hand in hand with its availability. We must therefore rely in encryption and secure authentication based technologies and protocols for the deployment of truly secure EHR in HIS. 


\subsubsection{HTTPS}

The Hypertext Transfer Protocol (HTTP) was originally created for the communication of hypertext documents between computers in the internet. The documents thus exchanged are easily viewed by anyone eavesdropping the communication. The Hypertext Transfer Protocol Secure (HTTPS) was created to ensure a reasonable protection by transporting the HTTP protocol over a secure (encrypted) connection, the Transport Layer Security (TLS).

The Web browser is originally provided with a set of Certificate Authorities (CA) that are trusted. Other CA may be added, by providing their certificates/public keys. From this point on, it can automatically identify a public-key because it is certified by one of the CA (it is signed by the CA, for which we have the public key), and thus establish a secure connection by exchanging one session key (symmetric) encrypted with the site public-key. We now have an encrypted connection with a trusted site. This trust is extended, that is, if we trust the CA, we trust the CA vouched entity for it being who it claims to be, in this case the website. The intranet in a Hospital can use this same mechanism to connect its machines or to receive external connections.

Connection security is of course as secure as the cipher used and the trustworthiness of the certificate, as well as the possibility to verify it, by checking the signature and even the revocation list online. This means that:

- We should trust the CA in order to connect to a computer, because most of the times the certificate is the only hint of a legitimate machine in the other side of a connection

- The type of encryption is hardly overstated. The initial connection is made with public key cryptography, which can be of various key lengths. Similarly, the symmetric key exchanged can also have various key lengths, thus these two keys determine the security of the connection, given the remote computer is legitimate.

Given these concepts, the actual use of this system is fairly transparent to the end user. All modern and popular web browsers support secure connections, which make web transactions much more secure. The server (remote machine) states the secure connection setup and the browser exchanges the keys needed, which may be done with "built in" certificates, or previously added/installed key pairs, such as public keys issued by a CA. This would mean that both machines and/or users could be uniquely identifiable. 


\subsubsection{Internet Protocol}

The security and overall performance of a network can be further improved with the use of IPv6 (Internet Protocol version 6). Since the internet is an organized system of selfgoverning networks, it needs a common voluntary protocol for interconnecting those networks [40]. IPv4 (Internet Protocol version 4) is currently the most used Internet Protocol. It has several drawbacks that are known for a few years, that have been addressed by the creation of IPv6, more than 10 years ago [41]. However, slow adoption is caused by the protocol not being mandatory, unawareness of its advantages, compatibility issues [40], as well as the fact that IPv4 still works well for the majority of uses.

\subsubsection{Role Based Access Control}

Traditional means of restricting system access to authorized users are Mandatory Access Control (MACC) and Discretionary Access Control (DAC) [42].

DAC is an access control mechanism that permits system users to allow or disallow other users access to objects under their control. The controls are discretionary in the sense that a subject with a certain access permission is capable of passing that permission on to any other subject (unless restrained by mandatory access control), and thus are allowed to grant/revoke access privileges to those objects under their control. From here we understand the difficulty in applying this paradigm to healthcare, in which the data is not "owned" by the user.

MACC is, on the other hand, defined as a means of restricting access to objects based on the sensitivity (as represented by a label) of the information contained in the objects and the formal authorization (i.e. clearance) of subjects to access information of such sensitivity. While with this method we are able to emulate functionality necessary to HIS, it is concerned more with access to information than functions. From this perspective, as healthcare personnel has distinct functions and perform distinct tasks in the production and access of data, it is as important to define those authorized functions along with access.

Role based access control (RBAC) is a newer alternative to the former [42], and is a policy neutral and flexible access control technology sufficiently powerful to simulate DAC and MAC. It permits assigning and updating use profiles seamlessly, as the user has its access rights inherited by its assigned roles, which can be added, changed and removed. This way RBAC allows for access control on HIS based not only on individual permissions, but also by assigning group policies based on the profession (such as doctor, nurse, etc.), specialization (orthopedic, pediatric, etc.), workplace (nursery, operating room, etc), and many other characteristics. This 
way, by enlisting a new cardiac surgeon in the organization, the system could automatically recognize its clearance level on the HIS, permitting and denying actions based on the expected pattern of use of the system. Information privacy and a much more fine grained access control is one of the main IT requirements leading to this solution [43]. One variation of this policy is Workgroup Based Access Control, in which we base access control in the workgroup, such as a clinical team, or a hospital ward.

As an example for the need for HIS integration, as well as the RBAC importance, a short hypothetical usage scenario is presented:

Mr. Doe has a comprehensive EHR in his local hospital. He is 64 years old, has a heart condition that requires permanent medication and has caused several hospitalizations in the past. When he is admitted on the hospital at $3 A M$, following shortness of breath and chest pain, the admission secretary, based on his credentials, has immediate access to his health insurance data, name and contacts, as well as a list of doctors who regularly take care of Mr. Doe. A triage nurse is immediately aware of Mr. Doe's Heart condition, by looking at recent admission and diagnosis data, and directs him to the cardiologist. After discarding an emergent condition, based on some tests and reviewing of the patient EHR, Dr. Logan, who is on call that night, admits him to the cardiology floor, where further workup will be held. The orderly sent with him checks the HIS and sees only his name, origin and destiny rooms. On arrival, he changes the location of the patient in the system to the cardiology floor. The secretary on the floor admits the patient based on the identification data and admitting doctor. The nurses can then start the prescribed medications and update the patient's condition at regular intervals. The next morning, in rounds, Dr. Alpert (cardiologist) orders some new tests, as he regularly sees Mr. Doe at his office. The technicians in charge of the CAT scan ordered know the patients identification, type of exam and what is to be inspected, so they can adjust the machine parameters. Dr. Self (Radiologist), after reviewing Mr. Doe's medical history and previous exams, reports a progressing stenosis on one of the cardiac vessels, and states clearly its location. Mr. Doe undergoes a procedure where the vessel is carefully dilated, with a coil placed on it so it stays that way. The medication is updated on the EHR and after two days Mr. Doe goes home, feeling a lot better. He has a follow up visit in just a few weeks, and when he files his discharge papers he is given a login and password so he can access (patient) all his medical information from the internet, along with a manual for the safe use of this PHR. He can view, but not alter anything on the pages, except for some of his personal data. 
Later in that week, the billing personnel accesses all expenses during Mr. Doe stay, in the form of tests, days on the floor, etc, that is, the minimum necessary for billing his health subsystem.

Dr. Smith, a resident on the Internal Medicine ward asks for permission to do a research on Chest Pain in the Emergency Room. After he receives approval of his work, he can temporarily access all patients admitted in the Emergency Room in the last year, anonymizing his findings for publication purposes.

This small example addresses the variety of healthcare and other personnel that can access a single EHR during a few hours. Role based access may give unrestricted access to some information, whilst withholding other. One person may even have two roles, as these have hierarchies, such as the doctor who is also on the board of administration.

While having the aforementioned advantages, RBAC is not a straightforward task to accomplish. In very large systems, managing these roles and their interrelationships is a formidable task that is often highly centralized and delegated to a small team of security administrators [43].

The MEDIS project (Serbia) [39] is a distributed patient record project, which holds authorization policies for hierarchy. It defines hierarchies of how, when, where, why and who attributes (hierarchy of roles, professions, regions, etc.). In that way, a different access control can be implemented - hierarchical RBAC, with constraints defined by security attributes (software security, physical security rating, etc.) and non-security attributes (profession, specialization, etc.). This example sets preference on standards on communication, with emphasis on security, as a keystone. It tries to achieve even better protection than other paradigms, while maintaining functionality.

\subsubsection{Digital Signing of Documents}

Documents can be electronically signed by the use of asymmetric cryptography (as stated in section 2.1.2 - Asymmetric Cryptography). Digital signing with current technologies is a very good way to assure documents integrity and authenticity, as well as provide liability for personnel actions. This is very important for HIS trustworthiness, as data may be transmitted over insecure channels in distributed environments where stakeholders have never physically met, but must nevertheless be able to trust each other.

There is a signature standard approved by the National Institute for Standards and Technology (NIST) [44] employing public key cryptography combined with a one-way hash 
function. This is supported by a Public Key Infrastructure (PKI), where each user has a public/private key pair: The public key is available to the world while the private key is only known by the user [45].

The signatures may themselves be certified by other entities, hence allowing the creation of trust structures. For example, a professional medical association might certify all doctors by signing their keys, and other clinical professionals could be similarly certified by their own regulatory bodies. This way someone may know that the signature really belongs to a certified doctor.

Electronically signed documents can, still, lose value of evidence because the security of cryptographic algorithms decreases in the course of time (see page 9, section 2.1 cryptography), given the obligation to archive patient files for many years after its creation, which poses a problem to these schemes [21]. This was addressed in long term application by the Archisig project [46] and papers addressed the role of digital notaries, confirming the signatures, and validating them, through resigning before the technology used initially is obsolete [47]. These frameworks are efficient and allow for the lifespan information need to be kept under the rule of law.

\subsubsection{XML Encryption}

XML (eXtensibile Markup Language) is a set of rules for encoding documents electronically. It is currently the most relevant standardization effort in the area of document representation through markup languages and is rapidly becoming a standard for data representation and exchange over the Web [48]. Various languages have been developed around this concept, including the ones used in productivity suites such as Microsoft Office ${ }^{\circledR}$ (OpenXML) and OpenOffice.org (OpenDocument).

XML-based security standards, which are paraphrased as XML security, are developed by the World Wide Web Consortium - W3C (http://www.w3.org/). XML security consists of three recommendations: $\mathrm{XML}$ (digital) signature, $\mathrm{XML}$ encryption and $\mathrm{XML}$ key management specification (XKMS), all of them published by the W3C, as free open standards [49].

$\mathrm{XML}$ encryption specifies a process for encrypting data and representing the result in XML. The data may be arbitrary data (including an XML document), an XML element, or XML element content. The result of encrypting data is an XML Encryption element which contains or references the cipher data. This is valuable to encrypt data in a HIS setting, especially when associated with digital signature. 


\subsection{Chapter Summary}

HIS are becoming more and more common, given the need to deal with increasing amounts of data, and to properly use it at the point of care.

Although this move is rather logical in the world we live in, and somewhat delayed concerning other society areas, such as banking, care must be exerted when planning it. There are various examples of neglected privacy in HIS in general and the related EHR in particular that should make us plan, test and deploy such systems with the care employed in any other critical systems, such as life support. We must ensure EHR is, at least, as secure as paper based records, but we should aim for higher security, as the current state of privacy can also be greatly improved.

There are many areas to consider: computer security, servers, network and communications, physical security and the end user. These are tasks hard to accomplish, and many problems have already arise from bad planning and/or misuse of HIS. Several steps must be taken to ensure technology is put to its best use to protect HIS data. The planning must have various approaches to the problem, in all these aspects. Current state of HIS security in Portugal is far from optimal.

Confidentiality, Integrity and Availability are the cornerstones of HIS security. A secure system must have strong authentication to allow for accountability while upholding confidentiality and integrity.

Password protection has proved itself flawed and breach prone. Biometrics are a valid authentication method, but there as various concerns about security, and they are expensive. Cryptography provides secure authentication and communication: A PKI is an established secure way to authenticate users that have never met in a network, beyond reasonable doubt, and can be used to communicate securely.

Healthcare information is private, and very important. So important that, more than two thousand years ago, it was considered in a sworn duty as a holy secret. This secrecy is the basis of clinical trust and is in the very foundation of good healthcare. That should be well understood when dealing with these privacy issues. 


\section{Smart Cards}

\section{State of the art}

Cards have come a long way since the introduction of the first plastic cards by Diners Club half a century ago. With patents holding the concept since 1968, although the technology to produce them was only available in 1976 [50], smart cards have a long history. France was an early adopter, namely in the financial institutions. By 1992, the magnetic stripe bank cards started to be replaced by smart cards for security reasons. Smartcards underwent major overhauls and the most recent are now more like mini computers, with the processing power of desktop computers from a few years ago. Smart cards are widely known for their flexible uses and strong security features.

\subsection{Smart Card Technology}

Smart cards are memory only or processor-enabled semiconductor based technology. For the scope of this work, we will consider mainly the latter, which constitute the majority of issued cards nowadays.

Smart cards generally contain a chip, of no more than $25 \mathrm{~mm}^{2}$, with a few hundred bytes to several kB (Kilo Bytes) of RAM, up to several hundred kB of memory and may also have special circuitry to perform cryptographic operations such as RSA public key encryption, signatures and verification. Some of the newest chips have 32bit microprocessors with 660kB EEPROM, $160 \mathrm{kB}$ ROM and 16kB RAM.

Smart cards are exceedingly common on mobile communications (SIM - Subscriber Identity Module - cards), being also increasingly present on banking, physical access control, network access control, transportation and digital identity [51], providing authentication, functioning as tokens, and controlling access to places and services.

The international standard ISO 7816 defines smart cards with contacts [52], but other standards (e.g. ISO 14443) exist, and those may have an interface by contact, contactless or both. Contactless and Near Field Communication (NFC) smart cards communicate with a card reader through radio frequency (RF) technology. The latter is a wireless interface and protocol, defined for consumer electronics [53]. Contactless smart cards can enable fast transactions with devices, and are used, for example, in mass transit systems (such as toll charge systems) and physical access control. 
Smart cards have bi-directional data flow: data is read from, as well as written to the card. In general, smart cards support the storage of information that can be "read-only," "addedonly," "updated-only," or not accessible. They can support the use of java-based [54] or other applications, as to enhance the flexibility and portability of applications for these devices, bypassing hardware or software limitations by the use virtual machines (VM). These virtual computers, with their particular software, run on "virtual" hardware emulated on the physical machine - that is, the host machine has a particular software which emulates an entirely different computer, with the ability to run different software as well as adding layers of security (VM's bypass some security issues, as well as having their own protection measures).

Processor-enabled smart card software is stored in permanent nonvolatile, read-only memory. Application data stored on the card is kept in Electronically Erasable Programmable Read-Only Memory (EEPROM). The contents of this memory may be erased and new data can be reloaded electronically and they have an embedded silicon-based 8, 16, or 32-bit processor, with even the 8-bit microprocessor-based smart card being almost as powerful as the desktop PCs of the early 1980s [50].

To communicate with a computer, smart cards need a smart card reader connected to the computer (usually through a USB - Universal Serial Bus - port), a driver that allows for the reader to function properly in the Operating System (OS) environment, and a piece of software called middleware, so that their protocol (language) may be translated and understood in a standard way by the computer applications. Some investigators posed the possibility of network enabled smart cards, which would obviate the need for middleware and function as a network computer, and these cards have already become a reality, with potential for commercial application [51]. The protocol specified by ISO 7816-4 for communication with the host is half-duplex, compared to the full-duplex protocols TCP/IP, and hence the need for middleware, adding to cost and inconvenience.

\subsubsection{Java Card}

Java, a programming language executed in a dedicated Virtual Machine (VM), making its writing platform independent, as gained significant share in computer applications. It is an "object-oriented, platform-independent, multithreaded programming environment. It is the foundation for Web and networked services, applications, platform-independent desktops, robotics, and other embedded devices" [55]. Pervasive to mobile technology, Java language, which is now open source, [56] has also entered smart card market, promising better compatibility, enhanced productivity and networking oriented, secure transactions. Java Card 
3 is the newest specification. It functions as a web server in a card, supporting HTTP and HTTPS, in both contactless and contact cards. It is also capable of running multiple communication interfaces, each with its own co-resident application. Java Cards have extensive security implementations [57], such as the possibility of utilizing RSA 4096 bit key pairs, and have already been certified with the highest security standards, such as parts of common criteria's Evaluation Assurance Level 7 [58] (see 3.3.1 - Evaluation/Certification) applied to the VM and java software.

This software runs on existing chips and ads new security layers, as the VM itself is protected. Given the high scrutiny involved in this widely used technology and the fact it is open source could help smart cards becoming truly network enabled secure devices, without the need for further middleware.

\subsection{Standards}

The best-known standard is ISO/IEC 7816, which applies to contact-based cards [59], managed by both the International Organization for Standardization (ISO) and the International Electrotechnical Commission (IEC). It consists in fifteen parts:

- Part 1: 7816-1 Physical characteristics;

- Part 2: 7816-2 Cards with contacts - dimensions and location of the contacts;

- Part 3: 7816-3: Cards with contacts - Electrical interface and transmission

- protocols;

- Part 4: 7816-4 Organization, security and commands for interchange;

- Part 5: 7816-5 Registration of application providers;

- Part 6: 7816-6 Inter-industry data elements for interchange;

- Part 7: 7916-7 Inter-industry commands for Structured Card Query Language (SCQL);

- Part 8: 7916-8 Commands for security operations;

- Part 9: 7916-9 Commands for card management;

- Part 10: Electronic signals and answer to reset for synchronous cards;

- Part 11: Personal verification through biometric methods;

- Part 12: Cards with contacts -- USB electrical interface and operating procedures;

- Part 13: Commands for application management in a multi-application environment;

- Part 15: Cryptographic information application.

The contactless standards include ISO 10536 for close coupled cards (working at approximately $0-1 \mathrm{~mm}$ from the reader), ISO 14443 governing proximity cards $(0-10 \mathrm{~cm}$ from 
the reader) and ISO 15693 covering vicinity cards and smart labels. The more common standard in contactless cards is ISO 14443 [60], which has four parts:

- Part 1 14443-1 Physical characteristics;

- Part 2 14443-2 Radio frequency power and signal interface;

- Part 3 14443-3 Initialization and anti-collision;

- Part 4 14443-4 Transmission protocol.

\subsection{Smart Card Security}

Security is the smart card industry main reason for existence. Cryptography implementation, secure data holding, and programming flexibility are assets built on a very strong security foundation [61].

A smart card takes advantage of state of the art silicon technologies, including a microprocessor, with or without dedicated coprocessors, to improve speed or security. It also holds analog parts and sensors with the intent of protecting it against attacks. Embedded software, based on secure operating systems, virtual machines, application firewalls and cryptography, make them highly secure devices [62]. The data stored on a smart card can be protected by active data encryption schemes and/or biometric identification. Access mechanisms are complex and based on software and hardware, trying to circumvent all kinds of possible faults and vulnerabilities [61]. Because of this, smart cards are extremely difficult to duplicate/clone.

Well known secret key algorithms are implemented in a number of smart cards, such as Data Encryption Standard (DES), Fast data Encipherment ALgorithm (FEAL), Advanced Encryption Standard (AES), as well as proprietary ones, namely in the telecom business, with fairly small and easy to manage keys (56 to 128 bit length). For higher end smart cards, public key algorithms, such as RSA (see corresponding section on 2.1.2) or DH (Diffie-Hellman) are available, with up to 2048 bit length keys [61]. Java card 3 even allows for 4096 bit RSA keys. One of the main advantages of utilizing a smart card to process public key pairs is that the private key never leaves the device, assuring its protection. Public key exchange of information allows for safe exchange of information over an insecure connection. Data may be eavesdropped, but not understood, or at least, not practically.

However, there are various attacks aimed at smart cards, namely by discovering their secret information or altering its use or function. Cryptanalysis, the art or science of analyzing 
and recovering secret information hidden in cryptography systems, can be separated is various fields, as shown in Figure 6.

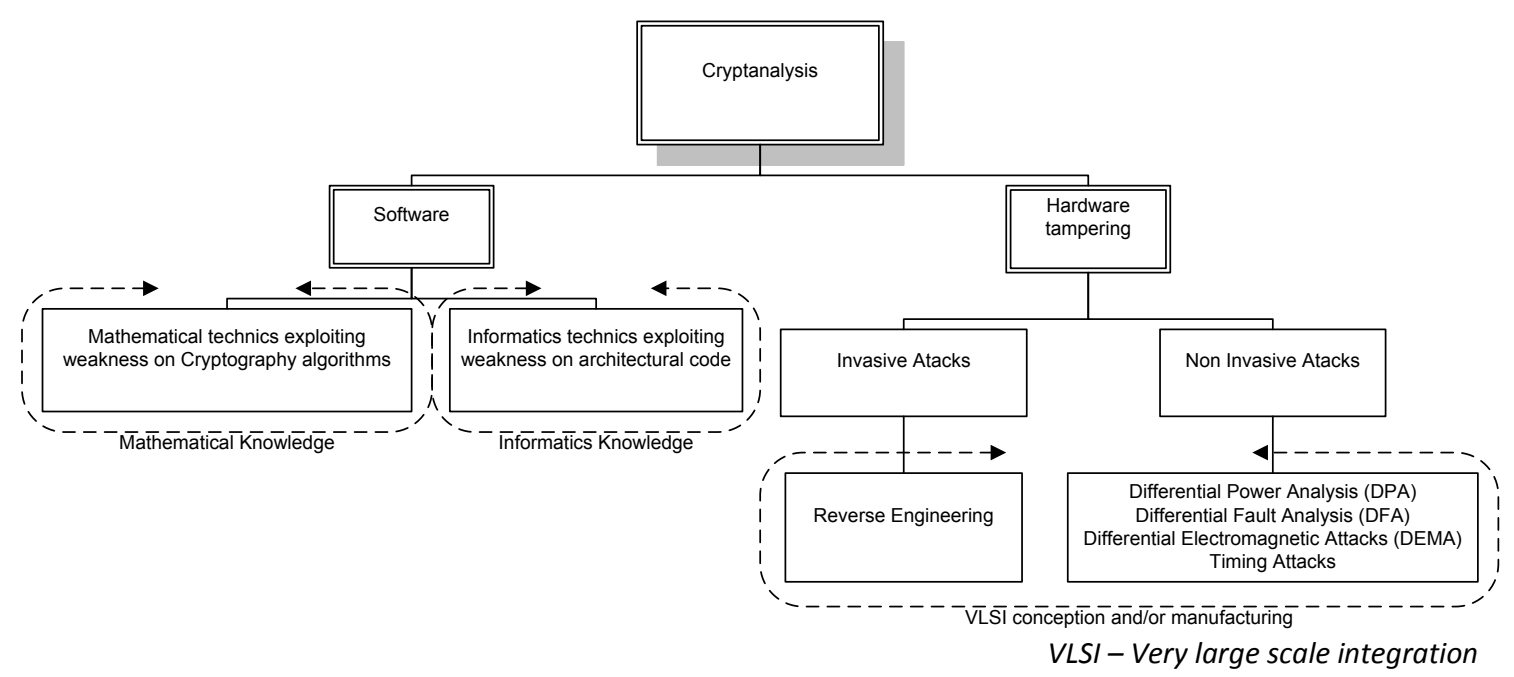

Figure 6: Cryptanalysis fields and some important attacks on smart cards

These attacks represent a true menace to smart card security, one that chipmakers try to circumvent in various ways. Recently, a security flaw was found on a chip, the "MiFare Classic", by Netherlands researchers [63]. This chip is used in public transportation cards, such as London's Oyster card, Netherland's OV-Chipcard, US Boston's CharlieCard, and in numerous wireless access control and ticketing systems worldwide. These cards sold around of 1 billion worldwide. This exploit was published after the researchers won a legal fight with the manufacturing company, NXT [64], on the right to publish their results. Their work used exploits on the protocol, but was built on the results from two Hackers, Karten Nohl and Henryk Plötz, who used invasive hardware based techniques to find the algorithm in which the card was based. In this case they did so, partially, by slicing the chip and taking pictures with a microscope. They then analyzed these pictures, looking for specific gates and connections, to reverse engineer their function, and thus reproduce the algorithm. Other work followed these findings [65], approaching algorithm weaknesses in other ways.

This exploit is based in a combination of invasive hardware attacks and subsequent exploit on software level. In this particular case, the security exploits are easy to replicate and require little equipment. Given the number of circulating cards, this is a good example of a major problem caused by poor design, but also because it is not always possible to preview new approaches used by hackers.

It is, still, possible to reduce the danger of compromise, by clearly identifying: 
- The assets to protect (secret keys, software, currency, etc.)

- Identify the information that may be misused or compromise security

- The types of attacks it must be able to withstand

- Limit bugs by extensive testing and quality assurance of both software and hardware; Create deep interaction and integration between software and hardware

- The insurance level of the embedded OS

- Third party evaluation and quality assurance

The security measures needed to create secure smart card technologies are fairly mature, with over 20 years of experience. Its successes and failures produced vast knowledge on the subject. The design must include functions for security (like random generator, specific local encryption engines, etc.), all functions involved or related to security must be implemented in a robust way (minimize leakage and sensitivity to disturb) and the functions and their implementation need to be proven for security [62]. A company must enforce high security levels at all development stages, using state of the art technologies, even in the way the card relates to a computer: A card must be able to trust and be trusted in a connection, the reason why public key cryptography is usually found in secure smart card products.

The smart card is gradually becoming even more secure with all the manufacturers developing and implementing state-of-the-art countermeasures for all known attacks both in software and in hardware. All this makes it the smartest, low cost, security token [61] (see 2.2.3 - Token authentication).

\subsubsection{Evaluation/Certification}

Certification gives people the confidence needed to use a system, if they ultimately trust a third party, the certifier. Independent certification, with proven impartiality, as well as thorough and reproducible methodology, is a well proven methodology for the establishment of trust.

The Common Criteria [66] was created by a group of nations, with the aim of having an international standard for security recognition. This way, IT products may be evaluated once, and the product of this evaluation can be accepted by other countries, and the criteria ensure that products relate to high and consistent standards, contributing significantly to confidence in their security. This is also known as standard ISO/IEC 15408. Essentially, this is a mean of 
comparing different independent evaluations, rovided they follow a rigid common set of requirements [67].

The result of Common Criteria evaluation is an Evaluation Assurance Level (EAL), ranging from 1 (less secure, low risk) to 7 (highest formal testing, very high security assets) [67].

\subsection{Smart Cards Use in Healthcare}

\section{The use of smart cards in healthcare around the world}

There are many examples of smart card use in HIS. Germany, France, Canada are some examples of big deployment projects. The Smart Card Alliance published a report on smart card usage in HIS, and its contribution to HIPAA compliance [68], in which they are pointed out as a practical solution for the majority of issues in this setting.

HIS need to grow outside institutions, they need to connect [69]. Electronic Health Records (EHR) should be easy to access, yet only by who has permission and is properly authenticated to do so. People need to know and trust their security $[70,71]$, hence the role for secure and strong authentication.

HIS can greatly benefit from public key cryptography. Users can authenticate themselves univocally, as well as sign documents in a liable way, with the possibility of doing it remotely $[38,72]$. A secure and pragmatic way of doing so is by the use of a PKI and smart cards.

Some countries use smart cards for multiple/various healthcare purposes. We will now review a few.

\subsubsection{Austria}

The Austrian Social Security Administration has replaced the existing paper-based system with eCards. The cards' functions include permitting holders to receive medical services and allowing entry to medical history and e-government. The cards will eventually evolve into citizen cards. Issuance and management of the 8 million cards is facilitated by Bell ID's ANDiS Management System. This manages the complete lifecycle of the cards with an integrated Global Platform based post-issuance personalization function. This allows cardholders to download and reload applications at a later date, and also enables the Main Association of Austrian Social Insurance Institutions to change on-card data via their own e-portal, the Karten Services Portal [73]. 
This project may not be fully compatible with the European Citizen Card (ECC) standards (CEN/TS 15480, among others) as these were finished after the initiated roll out [74].

\subsubsection{Canada}

A pilot study designed to evaluate the smart card use in the Canadian healthcare system and its findings were published in 2001 [75]. It served as a mobile repository in which pertinent information was stored. Patients have read only access, thus only professionals can enter relevant information in the card. 299 healthcare professionals and 7248 patients/clients were enrolled.

The results showed that a moderate advantage was perceived with the use of such technology, and high expectations could not be created, as the perceived advantage over the use of smart cards was lower in the groups that actually used it.

This study showed that professionals didn't feel that use of this type of information technology in the provisioning of medical services did not seem to improve the image of the health care professional, and that professional use of the system was not really influenced by the official position taken by its professional association.

\subsubsection{Denmark}

This country has deployed, since 2003, an ambitious Digital Signature program, in which crypto keys are distributed to Danish citizen's to allow for remote authentication into private and public entities. Nevertheless, as of April 2008, Denmark had no plans to issue card based eID's [76]. This is mere software based scheme, which is also used to access public services related to healthcare, such as appointments and health records. Although this is not related to a smart card deployment, it intends to depict the perceived importance of public key importance in government and healthcare, which use can be greatly enhanced by smart cards.

\subsubsection{Finland}

Finland Launched in 1999 an eID card (FINEID) featuring the holder's photograph and containing a microchip that stores the user's eNumber [77]. In addition to normal identification, the FINEID card can be used for electronic transactions and acts as an official travel document for Finnish citizens in the Nordic countries and 27 other European countries. Besides a secure network key for authentication on online services, the card nowadays (with more recent upgrades) can also be used to electronically sign documents and encrypt email messages. 
Since June 2004, citizens are able to include their health insurance data and therefore obviate the need to carry the Kela card, which was Finland's main healthcare identification.

The Kela card [78] contains information on the cardholder's health insurance, identification (including photograph on newer models) and may include other data:

- Indication of pensioner status

- indication of eligibility for pensioner discounts for public transport fares

- disease codes showing eligibility for special reimbursement of prescription drug costs

- $\mathrm{R}$ code (for those who have been issued a Front-Line Service or Veteran's Badge)

- $\quad M R$ code (for those who have participated in minefield clearance operations)

The Kela card does not contain a chip with the mentioned information, and cannot thus be considered secure.

\subsubsection{France}

The French SESAM-Vitale scheme was implemented in 1998 and had been successful in reducing administrative costs, cutting fraud and increasing payment efficiency. Today the program links more than 220,000 health care professionals with the health insurance system. It is based on the issuance of a health smart card to each French citizen aged over 16 years. This is the passport to access health care services and to receive insurance reimbursements. Each health care professional also has a professional card (the CPS smart card), which establishes the interface between the citizen who requires care and the professional who provides it. For example, the basic secure transaction process involves the two cards (both the VITALE patient card and the CPS professional card) to sign an electronic medical claim form. This is done either on the host computer at the doctor's office or directly by the portable terminal when visiting the patient at home. It is transmitted via the Internet for reimbursement. The country is now in a consolidation and renewal period with the Carte Vitale 2, a smart card with a new chip containing $32 \mathrm{~KB}$ of secured memory and up-to-date cryptography, standard ISO/IEC 7816 [73].

Le DMP (Dossier Médical Personnel - Personal Health Record) is another product for the facilitation of remote secure interaction via smart cards (see 4.4.5 - France) [79]. 


\subsubsection{Germany}

Germany was the first country to introduce a card into its healthcare system. It started to issue magnetic strip cards with a very limited dataset in 1994 [80]. The patient controls this limited information stored in the card, and can erase it at any time. The information is provided and updated by healthcare professionals only.

In 2003, the bIT4health (better IT for better health) project defined an architectural framework and a related security infrastructure for the German health telematics platform. A business analysis predicted that the replacement of the traditional paper based prescription by electronic means would save about 700 million Euros a year, which would render the initial investment profitable in 2 years. Furthermore, a reduction on medication errors or wrong information provided, could contribute to reduce some of the 25,000 deaths per year in German patients [81].

This project is probably the world's largest healthcare smart card initiative. Healthcare professionals are also issued professional cards, in a ambitious process: 80 million patients, 350,000 doctors and dentists, 2,000 hospitals and 22,000 pharmacies [73]. The smart card used is an active security device employed to perform strong authentication of the patient, in order to reduce fraud costs and increasing privacy. It provides an emergency data set (such as blood group, allergy alerts and ongoing treatments records), pointers to the patient's electronic health record (EHR) components distributed on the net, a medication file, immunization passport, provider's reports, patient's receipts, and finally and especially it can act as an electronic prescription carrier. The back of the card functions as an European Health Insurance card, which has replaced the E111 forms, and secures EU citizens basic medical care in other member states.

From a technical point of view, the card is a contact based smart card capable to process public key (PK) algorithms. The physical characteristics comply with ISO/IEC 7816-1[52] and related standards. An HPC is a normal size card (ID-001 card). Most of the EHR data will be server-based and accessed via information links on the card, due to the limited storage capacity [80].

Authorized health care professionals can read personal information only if the patient consents by entering the card's PIN. An estimated 80 million patient cards will be renewed over the next five years. The first health care pilot program began in December 2006, involving 70,000 units in seven regions. Definitive rollout started 2009, first in northern Germany. There have been, however, some issues that are hindering mass adoption. Namely, the equipment to 
read the cards is not mandatory to offices, clinics, pharmacies, etc., and its cost has to be, at least partially, supported by those [82].

Initial data on the possibilities and value of this new tool is encouraging [80], as this smart card generation, together with a standardized national telematics platform, will offer the chance to develop a broad range of applications for both the patient and the health care provider.

Recently, there was a setback with the CA (Certificate Authority) management. The Hardware Security Module (a particularly powerful security device that has its own processor and random-number generator) used to store the private keys for the root certificates suffered from an operational failure and, consequently, the private keys were completely lost, as there was no backup. The module responded to a supposed attack, as it should. The problem ensued because the company that stored it had a contractual agreement for not having a backup. This will result in the reissuing of the first generation cards on future stages of testing [83].

Some economic results of the program, as well as lessons learned from it, are depicted bellow[84].

\section{Economic Results}

- First year of annual net benefit, i.e. when annual benefits exceed annual costs: 2003, year 2

- Estimated annual net benefit for the year 2008: approximately $€ 450000$

- $\quad$ First year of cumulative net benefit: 2004, year 3

- Estimated cumulative benefit by 2008: approximately $€ 3.5$ million

- Cumulative investment costs, including operating expenditure, by 2008: approximately $€ 1.5$ million

- Estimated productivity gain, measured in decrease in unit costs of providing a patient with a valid and accepted insurance validation certificate for travel abroad: $65 \%$

- Distribution of benefits (direct positive gains) to 2008: Citizens - $96 \%$; Hospitals $-4 \%$

\section{Lessons learned}

- Clear, pragmatic goal for eHealth project, which addresses specific needs, is essential

- User-driven solution has a high probability for high acceptance rates: the application was developed for the benefit of citizens and hospital staff (eHealth can help overcome constraints of national borders) 
- A successful eHealth project is a series of continuous investments over time - an eHealth dynamic approach

- A successful eHealth project is part of a chain of activities, where there are many paradigms to change in preparation for the broad acceptance of such projects: Involve private and public companies (hospitals, pharmacies) as well as the public and government; Laws have to be prepared for the changes implemented, etc.

\subsubsection{Netherlands}

NICTIZ (National IT Institute f or Healthcare) designed a national infrastructure intended to make it possible for patient-related information to be accessed and supplemented from anywhere in the country, with real security concerns. Privacy is assured in a need to know basis, i.e. the information will be accessible only if needed for proper treatment. A dataset is already at place on several regions, allowing for data exchange on comorbidities and medications.

A smart card (UZI - Unique healthcare provider ID) is issued to health professionals as part of the IT security, to provide secure communication over the health network, facilitating encryption, digital signing and access logging.

A unique patient number is also at the core of the infrastructure, and Health Level 7 version 3 messages (HL7 v3 - see 4.2.4) is the communication protocol standard.

As intended by the NICTIZ, the authentication provided by the patient is to be based on a citizen card, but this functionality is not yet available. An electronic national ID card can provide a reliable method of allowing the patient access to all relevant information via the internet [85].

\subsubsection{Slovenia}

Slovenia initiated a nation-wide project to introduce smart cards in the health sector in 1995 and its full-scale deployment started in September 2000. This makes Slovenia one of the first countries, with France, to introduce microprocessor-based health cards.

The basic aim of the project was to support insurance related procedures, but the system was designed to be flexible enough and to be part of a national global health infrastructure. These cards are aimed for patient identification, authentication, storage of minimal datasets and as a European health card. 
During the initial six-year period of operation, the Health Insurance Institute of Slovenia [86] has gradually introduced new functionalities (e.g. ordering the European health insurance card at self-service terminals) and new datasets were included on the card (e.g. issued medication), improving the system's efficiency, number of applications and ease of use.

The content is only viewable by health professional's card holders, and holds electronic records of the following data items:

- card holder (name and surname, address, sex, date of birth);

- health insurance contribution obligor (registration number, title, address, type of contribution obligor);

- compulsory health insurance (validity data);

- $\quad$ voluntary health insurance (Insurance provider, type of policy, validity data);

- $\quad$ selected personal physician (general physician/pediatrician, dentist, gynecologist);

- issued medical technical aids;

- voluntary commitment to posthumously donate organs and tissues for transplants;

- Issued medication.

There are some technical components of the system noteworthy:

\section{Health insurance card}

The health insurance card is a microprocessor card with 16 kB memory capacity. Reading and modification of data on the card is subject to strict protection, requiring the health professional card and dedicated hardware and software. As of December 2007, 2.061.659 cards were in circulation.

\section{Health Professional Card}

Reading and recording of the health insurance card data is only possible with a card reader, in the presence of the health professional card, with the personal identification number (PIN) entered by the card holder. The system conforms to the health professional card concept being introduced in other European countries [87], such as France.

The health professional card allows its holder access to the data required for his/her work and that falls within the domain of his/her competencies. The card is issued to doctors, medical nurses, administrative officers in the reception offices, pharmacists, physiotherapists and other health care workers and authorized officers of the health insurance companies. There were 20.607 such cards in Slovenia in December 2007. 


\section{Self service Terminals}

There are 298 self-service terminals in operation, whose primary function is related with card updating. They are installed at public locations across Slovenia, in particular in hospitals, health centers, and health insurance company offices.

The initial cards were capable of symmetric cryptography. It was proposed [88] that only the professionals cards should be capable of producing digital signatures, in a nationwide upgrade for the health infrastructure, and as a way to reduce costs and simplify the upgrade.

This upgrade is happening [89] [87] and as stated in the Health Insurance Institute of Slovenia webpage [86]: "The final goal of the renovation is the transition from the current system, in the context of which data is exchanged between users in the healthcare system using the card as a data carrier, into a comprehensive on-line system where the card will serve merely as a key to access data stored on data servers."

As the new health insurance card will therefore give the cardholder access to his or her own data stored on the servers while giving health professionals access to insurance and personal medical data (upon agreement between the users of the system). The health professionals cards gains the new functionality of being able to authenticate the user online, to access remote information, as well as digital signing capabilities.

After completing this initial goal (the card containing pointers, instead of data, which are accessible online), there are other planned functionalities:

- Electronic prescription,

- Insurance holder's access to his own health and insurance-related data,

- Access to analytical data for health insurance purposes,

- Other electronic health insurance documents.

With this upgrade of the card, the Slovenian National Health Service expects to accelerate the creation and exchange of health records between healthcare providers.

In 2008, Gemalto signed a contract [90] for this new card, in which it will provide middleware, digital signature capabilities, software applications, applets and cards to the project: "This new electronic health card is the first of its kind in Europe to feature a Java public key infrastructure (PKI) that will further secure the online health system via a digital signature for health professionals." 


\subsubsection{Sweden}

Since 2002 all hospitals and primary care centers have been connected via Sjunet, the joint telecommunication network administered by the organisation Carelink (www.carelink.se) and dedicated to healthcare. It also links together county councils and regions, pharmacies and several other healthcare enterprises This is a physically independent private network, which allows for the secure and reliable exchange of confidential data, including images, and also incorporates the possibility for video conferencing [91].

On the 1st October 2005 the Swedish Government introduced the "official" elD card containing biometric data. The new national identity card (Nationellt Identitetskort) is not compulsory and does not replace previous paper ID cards [91]. This card is called "official" because there are other eID's, both smart cards and merely software based virtual cards(files stored in a hard-drive), issued by various entities, and sometimes accepted for the purpose of identification.

For the purpose of accessing web services related to healthcare, the citizen may use his eID card in some counties.

In $200642 \%$ of all prescriptions in Sweden were transferred from the doctor to the pharmacy electronically via Sjunet, or by using web based prescribing - eRecept, developed in coordination with the Apoteket, the Swedish National Organization of Pharmacies.

Some of the economic results and lessons learned from the implementation of ehealth in Sweden are depicted below [84].

\section{Economic results}

ePrescribing in the Stockholm County generates an estimated annual net economic benefit of over $€ 95 \mathrm{~m}$ in 2008. In 2005, it already had a net benefit of $€ 27 \mathrm{~m}$ [84]. It is worth to retain that overall expenditure with the system was about $€ 4 m$ for 2001-2008 period.

- First year of annual net benefit, i.e. when annual benefits exceed annual costs: 2005, year 5

- Estimated annual net benefit for the year 2008: approximately $€ 97$ million

- First year of cumulative net benefit: 2006, year 6

- Estimated cumulative benefit by 2008 : approximately $€ 330$ million

- Cumulative investment costs, including operating expenditure, by 2008: approximately $€ 155$ million

- Estimated productivity gain, measured in decrease in costs per prescription: $58 \%$ 
- Distribution of benefits to 2008: Citizens $-20 \%$; Hospitals $-80 \%$

\section{Lessons learned}

- Key driver for eRecept acceptance in Stockholm has been the awareness campaign initiated and sustained by the County Council and Apoteket.

- In Stockholm, the main success factor for eRecept is considered to be the high level of cooperation between all, the healthcare providers, the pharmacies and the County Council, who are involved in providing the service, especially the cooperation between the senders and receivers of eRecepts

- A good and clear implementation strategy connected to a national strategy for electronic prescribing and a process of continual review has led to faster up-take of electronic prescribing in the County Council. This clear strategy has resulted in reduced delays and good use of available resources during the implementation phase

- The success of eRecept is also attributable to professional project managers in the healthcare organizations and Apoteket.

\subsubsection{Taiwan}

Taiwan's National Health Insurance (NHI) is mandatory. Since 1999, new smart cards were launched as part of a project designed to provide better quality of services and to improve the effective use of medical resources. They provide identification for the $\mathrm{NHI}$, as well as some important information, such as important physician orders, prescriptions and drug allergies, vaccination records and organ donation notes. The IC has data divided in: Basic, Healthcare, a Medical and a Health Administration [92].

A Data Center was created for the purpose of ubiquity and consistency of patient records throughout hospitals and primary care providers in the country, although the project is expected to grow through phases, i.e. firstly to provide basic administrative (identification) data, then clinical data, and only afterwards the full extent of insurance, medical and public health administration data [93]. There is a Virtual Private Network (VPN) in which the data center is accessible, and medical institutions using the VPN system must first go through an electronic identification procedure to confirm a user's identity [94]. The internet may be used by those not covered by the VPN.

Technical teams were provided by the NHI for consultation purposes.

The first phase of the project was thwarted by some problems. Although hospitals were encouraged to adhere to the card implementation and use, there was a great diversity on the 
adoption of these systems, namely adaptation of the HIS to the card. The impact of such change was investigated by Liu et all [93], namely at two components:

\section{The effect of changes to the existing workflow of the medical services}

$60.6 \%$ of responding hospitals felt that the time for an outpatient service was prolonged, $34.6 \%$ remain unchanged and few (4.8\%) became shorter. Interruption of the service were also pointed: The major causes of problems were $\mathrm{NHI}-\mathrm{IC}$ cards themselves (31.2\%), failures in authentication (17.0\%), malfunctions in interfaces between HIS and card readers (15.8\%), and failures in card readers (15.3\%). About 5.6 and $2.9 \%$ of the problems were introduced by unfamiliarity with the operations of the NHI-IC card systems and others, respectively.

Overall, no hospital was found to strongly agree with the new system, and a few $(2,9 \%)$ strongly disagreed with the project, as impairing to the workflow was evident.

\section{The adoption capability of existing hospital information systems}

The complexity and financial burden of the adoption of the card was related to the size of HIS. Bigger hospitals were found to have greater adoption time spans.

The NHI claims that the public satisfaction with the system increased significantly to almost $80 \%$ nowadays [92].

Taiwan is one of the largest healthcare smart-card based solutions in the world, and lessons learned with its implementation are valuable, such as the need for a marketing strategy, security audits, a comprehensive HIS security plan and integration testing and acceptance procedures [68].

\subsection{Summary}

Smart cards are essentially small computers, designed to accomplish several security related tasks. They are used in many applications, such as mobile phones and satellite/cable TV. Their main advantages are to provide secure authentication and to be very difficult to copy. Being quite powerful and versatile, they are capable of performing several complex operations without endangering protected keys, as these keys are only used inside the chip, by its microprocessor.

There are various standards on smartcards, which are very important to ensure its carefree use everywhere, with the same level of security. 
Exploits and techniques to find them in smart cards must be known and planning for the protection of the card is necessary, in order to achieve good independent evaluation on its security. Smart cards are very secure tokens, which can be further protected by password or biometrics.

The adoption of a smart card for healthcare purposes has been long pursued by some countries. Although the implementation is costly and error prone, good planning, in a multiple approach (i.e., all the players and paradigms involved in the process) can ease the transition to a better system. There are few countries which pursued an eID card also used in healthcare, which could alleviate some of the costs involved.

The IT requirements to plan, test and roll out these projects is considerable, but, despite this, projections on savings (both less expenditure and more healthcare quality, leading to less collateral expenses) are very positive.

One of the main challenges is to promote adoption, both by citizens (patients and the private sector, necessary for the full use of these technologies. Some delays were also present in the adoption because of unplanned, unforeseen events, which must be considered in similar starting projects.

The secure identification/authentication of key players, such as the patient and doctor, is a recurring aspect of these projects, to prevent fraud and to ensure quality, non redundant care. PKI is largely used for this aspect. 


\section{Electronic Health Records}

\section{The power of information}

Health records (or clinical records) definition can be as broad as the purposes it serves: "to recall observations, to inform others, to instruct students, to gain knowledge, to monitor performance, and to justify interventions" [95].

\subsection{The Importance of Electronic Health Records}

The importance of medical records is best illustrated by the way it can be put into use:

- To recall observations

- Recalling past observations or conditions is very important as a clinician has multiple patients. Apparently unassociated conditions or current medications may be of great importance when prescribing, etc.

- To inform others

- As the process of diagnosis is complicated, and the quality of information at hand influences the quality of conclusions (be it written, told by the patient, etc), information passed by a colleague with training aids in the speed of treatment. The clinician can make deduction and reason on the basis of previous findings, instead of having to repeat the steps necessary to reach a correct diagnosis.

- To instruct students

- Medical knowledge is very vast. One of the ways medical students learn is by studying and discussing clinical cases, which makes it easier to develop clinical skills and to incorporate knowledge.

- To gain knowledge

- Progress in healthcare would not be possible without clinical studies, and the analysis of its findings. Medical knowledge began by basic observations on conditions and patients and evolved to complex clinical trials, in which control and manipulation of variables permits precise and accurate conclusions. All these findings are still valid, as even single case reports can be of use in rare conditions/disease presentations, or as a starting point to other, more complex and better designed studies, thus forming a pyramid of evidence, based on the recording of simple facts and its subsequent analysis. 
- To monitor performance

- The performance of a drug, a healthcare institution, a diagnostic test, etc., may be ascertained from the proper records of patients, from which we may try to extrapolate conclusions, given a correct methodology.

- To justify interventions

- Medical history can be used to justify, for instance, the use of a drug in light of a personal condition that precludes the use of others, or a more aggressive treatment plan when conservative ones failed, etc.

Electronic Health Records (EHR), computerized records, electronic charts, etc. refer to a medical record on digital format. Electronic records may be as simple as a scanned bitmap image of the actual paper record, or as advanced as a fully interactive, searchable file, with multiple data input yields (images, reports, free writing, selectable items, etc) [96].

To most people, information concerning its health is stored on the providers, scattered in all visited institutions, thus making it very difficult to have instantaneous access to all data and creating "information islands" [97]. The paradigm of health records on paper makes it even harder to access and distribute all information related to a given patient. Furthermore, the amount of data generated nowadays overwhelms traditional storage, especially because of imaging studies [98], not only in a physical way, but because of the growing difficulty in retrieving specific data in a timely fashion.

The Ideal situation would be to have a secure, ubiquitous, complete, reliable and interoperable medical record. This is very difficult to procure. Security is an mandatory issue, especially if ubiquity is a reality. The fact that a patient private, law protected, medical history can be accessed from everywhere in the internet makes the framework used for service implementation critical in terms of security and requiring services planning and testing, because there is a need to protect The EHR confidentiality and integrity, while making it available to the right people.

This is not to say the EHR must be managed uniquely by one application. There are reports that integration between dissimilar departmental systems, for instance, in larger institutions, is not only possible [99] but in some cases even desirable [100].

In many countries there are initiatives for building an integrated patient-centric EHR as well as initiatives for transnational integrations. MEDIS, in Serbia, is an example of such an attempt. It is based on public standards $[101,102]$ and gives special emphasis to security aspects of design and deployment [39]. 


\subsection{Standards}

There are various standards related to EHR and PHR, as well as some standard protocols that define transmission of health data or health reports, which are important in the broader definition of the EHR.

\subsubsection{CEN/TC 251 - EN 13606}

The Comité Européen de Normalisation European Standard (CEN/TC 251 - Health informatics; EN 13606) [102] "Health informatics - Electronic health record communication" is a high level template which provides a set of design decisions which can be used by system vendors to develop specific implementations for their customers, containing several parts:

- Part 1: Reference model (2007)

- Part 2: Archetypes interchange specification (2007)

- Part 3: Reference archetypes and term lists (2008)

- Part 4: Security (2007)

- Part 5: Interface specification (under development, ballot approved)

Its content builds on many years of openEHR work, and adopts many of its constructs in a simplified form that are perhaps better suited to the more simple architectures within most contemporary EHR systems [103].

\subsubsection{EN 12251}

EN 12251 [101] "Health informatics - Secure User Identification for Health Care Management and Security of Authentication by Passwords" defines authentication and access control mechanism for EHR, using public key certificates.

\subsubsection{DICOM}

DICOM (Digital Imaging and Communications in Medicine) is a standard originally created for the transmission of medical imaging studies. It was developed by the American College of Radiology (ACR) and National Electrical Manufacturers Association (NEMA). [104]. The third version, published in 1993, is updated in a regular basis, referring to the year of update. The last update is DICOM 2007 version [105]. Given its purpose, DICOM standard has many limitations complying with all the needs of electronic records, but plays an important role in Picture Archiving and Communication Systems (PACS), and sometimes implemented in parallel and interoperable with EHR. 


\subsubsection{HL7}

HL7 (Health Level Seven) [106] is a nonprofit organization, accredited by the American National Standards Institute (ANSI) and other standards organizations, involved in the development of international healthcare standards. It presents a framework for the exchange, integration, sharing and retrieval of electronic health information, both clinical and administrative, which is widely implemented It is involved in the HIPAA [14] legislation since 1996, and collaborates with other healthcare standards groups, such as DICOM, CEN/TC 251 and ISO 215.

It provides:

- A messaging Standard ( $\mathrm{v} 3.0$ is the most recent)

- The workhorse of the standards provided

- The Clinical Document Architecture

- Provides an exchange model for clinical documents

- A clinical Context Management Specification (v1.3 is the most recent)

- The Arden Syntax for Medical Logic Systems (v2.0)

\subsubsection{ISO/TC 215}

ISO/TC 215 [107] is an International Organization for Standardization Standard on Health Informatics providing technical specifications for EHR. It consists on several working groups:

- WG 1: Data structure

- WG 2: Messaging and communications

- WG 3: Health Concept Representation

- WG 4: Security

- WG 5: Health Cards

- WG 6: Pharmacy and Medication

- WG 7: Devices

- WG 8: Business requirements for Electronic Health Records

Several standards have been created within this framework, including ISO 18308, which describes EHR architectures.

\subsubsection{GEHR/openEHR}

The GEHR/openEHR was first started in 1992 as Good European Health Record. The initiative was later named Good Electronic Health Record. It is maintained by the openEHR 
Foundation, non-profit organization defining itself as "an international, online community whose aim is to promote and facilitate progress towards electronic healthcare records of high quality, to support the needs of patients and clinicians everywhere". The innovative vision of this standard is the introduction of an archetype concept, in a two level methodology to model the EHR. The first level is more general and stable, the second more healthcare and application specific. These are modeled as archetypes, constraint rules that specialize the generic data structures that can be implemented using the reference model. Archetypes are defined by descriptive data, constraint rules, and ontological definitions [108].

Archetypes are models of clinical (or other domain specific) concepts. Technically they are formal specifications of clinical content, and clinically they are the basis to intuitively define, discuss and present clinical content. Existent archetypes may be used by many institutions but they can also be created [109].

The work done over the years was put into the development of standards such as the discussed EN13606 [110].

\subsection{Ownership of Data}

Ownership of clinical data is prone to several misconceptions. Depending on the place it is gathered, it may have different exclusive owners or be subject to a "joint ownership". The information may either be regarded as property of the one who gives, receives or pays for care. This leads to confusion on rights to access, change, destroy or transport any given clinical information. There are major differences in the way this type of information is ruled in the USA and EU. And within these legislations on/or recommendations, confusion arises in this era of digital information.

This matter is of great importance. It ultimately implies ethical and legal issues that are often debated, such as informed consent, clinical studies and trials, use of clinical information for insurance purposes (acceptance or rejection), marketing, etc.

Ownership of data also relates to intellectual property. Free circulation of health Information is only of interest when its source is reliable. Documents digitally signed could enhance the provision of intellectual property rights [47] (although without cost or earnings) as well as provide basis for trust in information produced in other locations. 


\subsubsection{USA}

Ownership of paper records is not debated, it belongs to the doctor or clinic that collects and maintains it. It can be sold or given to other clinicians for treatment purposes. Patients have the right to privacy on that information and access, but Federal and state laws do not extend these rights [111]. They cannot destroy this information, as an example. Beyond this interpretation of the law, digital information is much more difficult to rule, as is interpreting the existent rulings.

There are other legal regimes in the USA that apply to this type of Information: Privacy law extends the right to block access to the pertaining information, but not to really own it; Property law does not give rights over information, as it would impair development and interfere with other fundamental rights, although common exceptions are patents and copyrights, to a degree.

Although interoperable EHR would benefit most doctors, clinics and patients, and federal funding to integrate medical records exists and is often applied, these overlapping rights and confusion, along with the multitude of players involved (clinical personnel, clinics, insurance, state, patients), encourage the caregivers to withhold the share of information they control, in order to better preserve their business interests.

\subsubsection{EU/Portugal}

In the EU, personal data is protected by the EU directive 95/46/CE [15]. Individual member states adopt these rulings and may better define its conditions. Portugal adopted this ruling in the Law 67/98[112].

- Processing of racial or ethnic origin, political opinions, religious or philosophical beliefs, trade-union membership and data concerning health or sex life is prohibited, although safeguards are in place, such as consent to do so, medical reasons (in the interest of the subject), etc.

- Information shall be given to the subject to which personal data pertains about the identity of the holder of such information, the purposes of data collection, obligation or voluntarism on collection, right to access and rectify data, among others.

- There is the right to access and/or rectify data, as well as confidentiality and security of processing and right to erasure in certain conditions (such as no compliance with dispositions or rightful objection on the half of the subject). 
The law 12/2005 [16], in Portugal, defines the legal ruling of health information. It clearly establishes the patient/subject of data as the rightful proprietary of such data, and the healthcare providers as holders of that information.

- The patient has the right to know all contents of its health record, by means of a doctor, chosen by him, and to appoint someone of trust to receive this information. The law 46/2007 [113] altered the need for a doctor to mediate, not needed anymore in public institutions, but still on private practice.

- Data holders have the obligation to protect all data from unlawful use, access, violation or destruction.

- Data, after anonimization, can be used for research purposes.

- Data can only be imputed to or consulted by a doctor directly involved in the care giving process, or someone under his supervision, and equally obliged to ethical secrecy.

While not literal on timeframes, this ruling entitles a patient prompt access to his health data.

The necessity of a doctor/health professional to convey the information is related to the need of conveying subjective information, which can be hard to interpret or harmful (such as psychiatric notes on a patient). The Portuguese Medical Association Code of Ethics (2008) also states that the written memory (subjective) of the doctor is his intellectual property, but still states the patient must have access to all information in the record. This difficulty in defining what healthcare information is, and what to show the patient, is apparent in the Opinion emitted by the Portuguese National Council on Ethics and Life Sciences on the law project 788/X [114]: “(...) the way the patient should have access to the clinical record needs careful evaluation. It is not less certain that the clinical record, as such, should not be handed to the patient. (...) it is (also) an work instrument for doctors and nurses."

\subsection{Examples of EHR Implementation}

The ambulatory setting accounts for the majority of EHR use worldwide, in which the USA and Canada adoption rate (20-30\%) lag notably behind the United Kingdom (>90\%), Netherlands (90\%), Germany (42-90\%) and Australia (80-90\%). In hospital settings, the adoption is much lower overall, ranging from 5 to $10 \%$ in these countries [115].

There are some published experiences on this matter. This is not an extensive list, but it provides some typical examples: 


\subsubsection{Australia}

"Healthconnect" aggregates centrally summaries of records taken locally. This information is selected by patients and providers, by relevance. This system is expected to save AUD \$300 million on reduction of error and duplication of effort [116]. A New South Wales pilot project is encouraging hospitals and community providers to exchange clinical data for some 50,000 patients [117].

\subsubsection{Canada}

Canada created Infoway, a strategy to promote adoption of EHR among health providers, with the objective of health data exchange, namely the implementation of an interoperable EHR for $50 \%$ of Canadians by 2010 [117]. It is currently in the process of developing privacy and security standards and mechanisms for that purpose. Despite this effort, there is yet poor adoption of EHR among players for clinical information sharing, such as hospitals, which hinders this process.

\subsubsection{Germany}

Following the deployment of its healthcare smart card, Germany also plans for an EHR, based on the security provided by the card. Most of the EHR data will be server-based and accessed via information links on the card, due to the limited storage capacity [80]. Authorized health care professionals can read personal information only if the patient consents by entering the card's PIN.

The EHR where the card will be based is intended to comply with the revised CEN ENV 13606 "health informatics- electronic healthcare record communication", or its new name EN 13606 "health informatics - electronic health records communication" [103]. This aims to ensure trouble free interoperability.

\subsubsection{Netherlands}

The AORTA program addresses the basic infrastructures linking patients, care professionals and health-care insurers. AORTA is the Dutch national infrastructure for the exchange of data between healthcare providers. The focus of this program is to facilitate the realization of a national "continuity of care" oriented EHR. AORTA uses HL7 version 3 messages and documents as its core mechanism for information exchange [118]. 


\subsubsection{France}

Le DMP (Dossier Médical Personnel - Personal Health Record) is a product for the facilitation of remote secure interaction via smart cards. It allows a patient willing to enroll a way to access information related to his health, provided by multiple caregivers. It also allows him to manage access rights for health professionals. These see only information relevant to their profession. The information is stored remotely in a state-approved healthcare data hosting service, and the access is based on the CPS and Vitale card, in an encrypted format. A login/password access for the patient, from home, is being prepared. The system provides logging of the access, as well as a "break the glass" feature, which provides for an emergency access to the health information (with no prior authorization), which has to be later justified [36]. The DMP is also not used for epidemiological research and has protect access in various conditions, such as that its access cannot be used as a condition for any kind of contract (such as health insurance). The patient has the possibility of hiding some of the information from one or all doctors, a functionality that has caused some agitation among doctors [79].

\subsubsection{Portugal}

In the Madeira Digital (Digital Madeira) context, the digitalization of clinical information was the main objective, as well as the creation of a EHR for the region. The goal was to provide access to relevant clinical data from every point of care in a public clinical setting. A centralized architecture was created, connecting 3 hospitals and 54 general practice locations $[119,120]$.

The Aveiro Digital program (Digital Aveiro) promoted the Rede Telemática da Saúde (RTS Health Telematic Network). This network utilizes RIS for interconnection and is also a central repository of information [119].

Several other projects are being developed locally, namely in the two major hospitals of Oporto, with the objective of creating an EHR. The Centro Hospitalar do Porto is working with the Minho University, developing products for information collection and integration[121]. São João Hospital is using a system developed in the Oporto University (Faculty of Medicine), VCintegrator, which creates a virtual EHR, based on data collected in multiple information systems already in place, with the latest reports and clinical history, into a usable and accessible EHR [122].

The first applications with the purpose of substituting of paper based records are in cuurent use for more than 10 years and were created by a state institute, the now extint IGIF Instituto de Gestão Informática e Financeira da Saúde (Institute For the Health Informatics and 
Financial Management). Two programs were initially roled out: SAM - Sistema de Apoio ao Médico (Doctor Support System) and SONHO/SINUS - Information Systems directed respectively at hospitals and general practice units. These programs were followed by SAPE Sistema de Apoio à Prática de Enfermagem (Support System for Nursing Paractice). Most public health Institutions use them, as they are required for payment purposes. These programs were subject of very little upgrading since their creation, which may have led many hospitals to start creating/expanding their own systems.

\subsubsection{USA}

The Veterans Health Administration (VHA) is the largest integrated health-care system in the USA, and provides public-sector care for honorably discharged veterans of the US armed forces. Its performance has been often favorably compared to the private sector, in terms of performance and quality of care [3]. The Department of Veterans Affairs has, since 1999, a networked EHR and an associated PHR, which subscribers and staff can access. It comprises, among other fields, medical charting, provider orders and patient progress notes. This EHR has an estimated annual cost of US\$90 per patient and is easily accessible from physicians' offices or from the patient's bedside. It consistuted a step ahead from the availability of $60 \%$ of the relevant clinical information at the point of clinical encounter in 1995 to close to $100 \%$ in 2004 [3].

Kaiser Permanente, the largest nonprofit health management organization in the United States, introduced an EHR in 1999. The findings of a 2005 retrospective review were that several flaws in the implementation hindered easy initial adoption, and adaptation to the electronic environment was not straightforward [123]. Some aspects perceivably improved in this context, such as primary care visits reduction with the HealthConnect online secure messaging system, evaluated in 2007 [124].

Kaiser Permanente has more than $\mathbf{2 . 2}$ million of its members actively using its personal health record called My Health Manager. Their HealthConnect EHR, is stated to cover 8.7 million members, making it the largest privately funded EHR initiative [125].

The fragmentation of health data in the USA is a result of the multiple caregivers they can choose from, as there are many more EHR scattered throughout them. Interoperability between these systems is regarded as of extreme importance [117], both for economical and health reasons. 


\subsubsection{UK}

In this country, the EHR is also evolving at a national level: "NHS Connecting for Health is part of the wider NHS. It helps the NHS to deliver better and safer care to patients, via new IT systems that link General Practicioners and community services to hospitals." [126].

The 2003 National Health Service (NHS) contract with General Practitioners (GPs) [117] put in place large financial incentives for meeting quality standards, which has driven up the use of more sophisticated EHR systems with decision support. Automatic transmission of electronic prescriptions to pharmacies was also one of the objectives. This initiative is using openEHR archetypes and templates to provide a common and agreed clinical content on which to base its clinical applications, as part of standard CEN 13606 [110].

HealthSpace is a free, secure online personal health organizer, provided by the NHS, which aims to help anyone living in England, aged 16 or over, to manage their health, store important health information or find out about NHS services near them. It contains an area for online storage of important medical information, available to some citizens (as the service is just starting) [127].

\subsection{Health 2.0}

\section{Next generation on healthcare}

Despite the critical importance health has in people lives, business processes associated with health services are lagging behind the services provided by banks, travel agencies, insurance companies, etc. The electronic automation and online business models have not encountered, as of yet, wide adoption in this healthcare [128].

The reduction of error and increased safety are major drivers for adoption of electronic services, such as Electronic Health Records (EHR), but other arguments, such as services integration, records ubiquity, improved resources efficiency, as well as costs control and easier accounting, come into place, not necessarily in this order.

There are some studies $[129,130]$ that evaluate the impact EHR can have in health practice, and it seems clear that this is not a question of "if", but "when" will EHR become a critical commodity. Electronic records a necessity in today's ever increasing multitude of health records, coming from multiple providers. The paper paradigm is not able to cope with this massive influx of information that is being produced the most recent diagnostic methodologies, not to mention its inherent lack of flexibility. The ownership of the records 
(attributed to the patient/client) also imposes the flexibility of an easy data sharing model, rather than the more traditional data owning model.

Several surveys $[131,132]$ have shown that consumers are supportive of the notion of ubiquitous health records. Their major concern is the security of such records. Many patients already have some kind of personal health records, which varies from a drawer, full of medical exams and reports, to a more complex computer program or file, containing more organized information.

As the move to the EHR is unstoppable, the need to make the right decisions is upon the government, private companies, providers and the public. If a model is adopted and worked upon, in a collaborative effort including the healthcare professionals and patients, the EHR may be a reality well beyond what is being currently deployed in major health institutions.

\subsubsection{The challenges of Electronic Health Records}

The EHR is constituted by a longitudinal collection of electronic health information about individuals and populations [116]. Information Technologies (IT) are being employed to reduce medical error, as well as facilitate care. They are being advocated by the Institute of Medicine has a priority reform and a national commitment for today's health practice. Information technology plays a fundamental role to "support healthcare delivery, consumer health, quality measurement and improvement, public accountability, clinical and health services research, and clinical education" [133].

Reducing handwritten data reduces errors in interpretation and dramatically improves the storage/retrieval of information. The change from an in-patient to a more episodic ambulatory approach in today's healthcare mandates the need for easily accessible records. The need for risk assessment, billing, report creation/issuing and clinical research are not compatible with thousands of bulky charts hidden in giant basement warehouses.

The principles of confidentiality are often pointed out by patients, who want to know what information about them is stored, how will it be used and by whom.

Although difficult to implement, there is some consensus on the inevitability of cross institutional records, mainly because of regulations. In such a scenario, key points must satisfied for the sake of function, regulations compliance, acceptability (by both the health personnel and public) and still be able to make an evolutionary step, making it easier to compile, consult, analyze and distribute health records. There are also many strong economic 
(and other) reasons that will make it inevitable [134]. Creating and deploying a networked EHR should abide to several key points:

- The need for full disclosure: Confidentiality is an important subject when it comes to health. Patients will not disclose some information they feel that, for example, may be known outside the patient/doctor relationship. They may not want to disclose any information regarding their health to anyone other than the staff that directly provides care. However, this is a very sensible subject because of the need, for instance, for paying for a treatment, that may be directly associated with a condition, and still must be disclosed (to a broader circle of staff) because it directly implies materials, hours of doctors and or nurses, room occupancy, laundry, etc.

- Legal and ethical obligations: providers are subject to regulations and trust principles with their clients/patients. They have to document every visit, and must do so in a easy, quick way, so that they won't spend more time typing than actually examining and talking to the patient. One should not expect a nurse or physician to code everything in drop down and check boxes. In the USA, improper record keeping (illegible, inaccurate, or incomplete) may give rise for medical malpractice liability. There is an inherent risk to the use of early adoption, unrefined software. There's also the fear of malicious data manipulation [135].

- Cost: Substantial investment is needed for global approaches to HIS. Some drawbacks concerning the development costs of such systems are expected, and some examples have happen in major economies, such as the USA and the lack of proper financing following a promised health care technology development program (2004)[136].

- Readjustment: There is a need for paradigm shift with EHR and PHR. Some learning will be necessary and many steps taken today in the process of organizing a clinical record will/should become obsolete. Besides the costs involved, there is a learning curve to account for.

- Proprietary technology dependence: There is concern about reliance on proprietary technology/ software for such an important feature of a health system. Once deployed, the framework should be self sustainable, without worries of monopoly on the software and hardware needed for interoperability [19]. Public standards and/or technology would obviate this problem. The software and hardware should be patent free, fully based on open standards. 
Some important contributions for the clarification of EHR have been made by several entities. Markle Foundation funds the Connecting for Health, a major public-private collaborative, and its working groups issued comprehensive papers on the EHR and PHR [137139]. The EHR collaborative group consists of some of the most important stakeholders in the USA, such as the American Medical Association (AMA), the American Health Information Management Association (AHIMA) and the Healthcare Information and Management Systems Society.

OpenEHR [103] (see 4.2.6) is a global effort for the development of an interoperable EHR, maintained by the openEHR foundation. It builds on the research done for over 15 years, including the GEHR, by its members from an international community. The openEHR approach asserts that it is not the application, but rather the data (or health information), which makes up the health record. And further, that it is a common and agreed structure of that data that makes the electronic health record of any use for computing in healthcare. OpenEHR states a set of specifications for EHR, but not the software itself:

"The openEHR endeavour is about creating specifications, open source software and tools in the technical space for [a health computing] platform. In the clinical space, it is about creating high-quality, re-usable clinical models of content and process - known as archetypes along with formal interfaces to terminology. The design purpose is to enable semantic interoperability of health information between, and within, EHR systems." [140]

A web-based review and validation process for archetypes was also created, enabling international involvement and ensuring that released archetypes are of high clinical and technical quality [109].

\subsubsection{The concept of Personal Health Record}

Health systems fragmentation, leads inevitably to a multitude of data dispersed among several offices, clinics or hospitals. This problem can be addressed by a patient controlled approach, per se, or as part of a broader framework.

Personal Health Records (PHR), as opposed to Electronic Health Records, emphasizes the patient side and interest in this type of data. Rather than reporting to ownership, as most countries recognize all health data as legally owned by the patient, the nomenclature refers to the physical possession/accessibility of these records, as well as the possibility of interacting with them, adding or editing data, as well as to show them to whoever they wish. 
In a survey, Kaiser Permanente concluded that the majority (51\%) of Americans think IT should be a top priority in the next presidential administration, as well as $65 \%$ have gone online learning on a medical condition, and $47 \%$ preferred doctors who used EHR. On the other hand, concerns on privacy were also stressed by the results [131].

PHR can be held on paper, a portable media/device, a server with internet access, a PC, or a combination of those. It should, however, have a minimum set of data elements to insure interoperability [141].

The internet alternative to the more classical models can be supported, or funded, by governments, or simply accepted as a mean of integration of the EHR, one that can be controlled by the patient.

There are several companies that offer such service for a fee, providing the framework and storage. Some examples are: TeleMedical.com [142]; PersonalMD [143]; Medefile [144]; CAPmed [145]; My Medical Records [146]; mediKEEPER [147]; VIM Vault [148]. The latter is part of a product range oriented to all players in healthcare providing. As these are all based in proprietary technology, the lock down could have deleterious effects [149], putting the information at stake in case the company stops fulfilling its role.

These companies offer various levels of integration, types of access, different input/output methods and storage/retrieval functions. They are sometimes immature, limited in function, and some companies tend to end their services prematurely $[120,150]$.

\section{The Google approach}

Google released a PHR in 2008, with no fees, that aggregates different types of information, namely patient, doctor and clinic/hospital produced, in a secure, widely accessible manner [151].

Multiple profiles can be created and stored in the same account (which is the same account used for other Google services, such as email). These profiles can be linked to healthcare care providers, so they can read from and write to the account. Google also permits interactivity with partners, that is, they can expand the functionalities of the framework in proprietary sites. A list of partners from which clinical data can be imported from or exported to is available in the service page menu.

The information entered by the user is then done manually, that is, results aren't copied (as in a scan), or free text, but instead there is input for a condition, medication, or test result, 
selected from a list, and then the results are added, as well as the date and notes. For interoperability purposes, this eases import-export of data, since all fields are standardized and nomenclature is rigid (selectable, not writable). The profiles are also completed with personal data, such as age, weight, height, etc. There is, however, the possibility of uploading files and images, and this can include the scanning of paper exams.

Fields can be searched for more information, such as medication and diagnosis, so that the patient can learn more about his illness or about possible secondary effects/interactions with the drugs prescribed, or even search for a medical doctor. This feature, deeply related with Google's nature as a search engine, is intuitive and easy to use.

There's automatic flagging off possible interactions based on the prescription drugs entered on the respective field, and some charts can be automatically created.

The service is compatible with any computer with a current web browser and is free for private and professional entities. Google claims the information stored in their servers is well protected, with extensive software, hardware and other physical protections, as well as redundancy and strict backup policies.

The privacy policy is the same as the company's other products, that is, a lot of information, statistics and trends are stored and analyzed, but not in a way that can be traced to the user.

This service is already online, although it only is adapted and available for people living in the USA (partners, language, etc.). Some concern arises over the quality of information stored with this service. There are reports of billing information being utilized for filling the PHR (by partners). Inaccuracies appear to exist in that information, which is not always produced by health personnel (serves insurance claims), and is sometimes inherently inaccurate because of bad code choosing or inexistence of coding for the specific problem. One specific case involves inaccurate and/or misleading information from billing data from a hospital that ironically has a very good EHR accessible to patients [152].

\section{The Microsoft service}

Microsoft uses a somewhat different approach [153] for PHR. Besides planning interaction with hospitals and clinics, the same way Google does, it will work with some of the programs and web sites already focused on the PHR, such as the already mentioned mediKEEPER [147], and some brands of medical devices that send data to a computer, such as heart rate, blood pressure or glucose monitors. It also interacts with a "Vista gadget" that allows upload of 
information by simply adding it in a piece of software that sits in the desktop. These features are integrated in a "connection center" that provides interaction between these sources, and then updates the PHR.

There is also the possibility of uploading documents, rather than just imputing information, that are stored in a web server. There is a search service, similar to Google's, in which the users can learn more about their illnesses or medications.

The sharing of a record can be done in different ways: limited time View-only; limited time view and modify; custodial access (no time limit, full control). Privacy concerns are addressed with the promise that no private data will be used for commercial purposes unless the user specifically authorizes it.

The service doesn't allow at the time an account to be created from outside the USA.

Kaiser Permanente is working with Microsoft in a pilot project that will bring Kaiser's PHR and HealthVault together for Kaiser employees and their doctors [131].

\section{The catch?}

Google Health, as well as Microsoft's HealthVault is intended to give patients control of their health records, giving them the opportunity to have their records securely stored, in an easily, organized and always accessible way. Contrary to most other alternatives already in the market, Google and Microsoft state that security concerns are well covered, and no cost is associated with the service.

By supporting the upfront, development and maintenance costs on their structure, These companies are effectively making a sonant proposal, although some other problems are expected, also commonly encountered in the roll-out of EHR's [139]. Standardization of systems, interconnectivity and data, is a major problem, as the network will only grow as more people are served by it and this will only happen if health providers find it useful and easy to use the system (or are otherwise compelled by patients). The need to exchange data and the inherent difficulty and dangers has thwarted many attempts to create viable electronic health record projects.

The fact that these are private companies also rises some concerns, as many experts [19, 154] feel this data should always be on governmental/public domain, be based on open standards, and thus independent from single companies that could end support or stop the development of such important frameworks. 
How will Google and Microsoft handle all the problems previously encountered, especially security concerns? How will the ownership of data be dealt with? What kind of flexibility will be offered by these frameworks? As the services are fairly recent and not yet available from outside the USA, there isn't much information and/or experience to fully answer these questions. Google and Microsoft claim the information is private and any analysis on it is anonymous and automated, but, given the importance of information stored, it is difficult to understand if such products, at their current state, could abide to European law.

Google is a private company, whose core business is essentially advertising. Many feel that Google's data mining processes, despite their excellent services, are intrusive and overly personal. Some feel that Google is getting more and more of our inner selves in the virtual space [155], and privacy is becoming an issue when most of our lives can become accommodated in some hard drive from across the planet.

The same can be said about to Microsoft, which can be arguably trying to gain control over a niche of great importance invested by Google. This great importance, as Google puts it, is trust. People trust the companies who handle their health, because it is such a sensitive matter, and may use the rest of the products that company has, based on that same level of trust.

As far as relevant advertising goes, widely used on Google mail, the company claims search results won't be delivered based on information from Google Health, and Google health (at least currently) has no adds.

As some of these concerns are coherent with the problems faced by EHR in general, namely the difficulties with authentication and data protection, the same solutions apply, at least as long as these companies facilitate the process.

\subsection{Chapter Summary}

EHR may well be an important step both from a corporative point a view and in the lives of patients. It is important to have bedside information available to make important decisions; it could help save a great deal of money, by reducing redundant expenditures and accelerating care; health research could be improved. If we expand such an idea to a networked EHR/PHR, the possibilities would increase greatly, with many added benefits: It would facilitate patient's access to other doctors, who would be able to see the "whole picture"; it could greatly enhance telemedicine applications and all its gains in terms of healthcare access; it could 
enhance GP's performance, as true family doctors, in their pivotal role in the SNS healthcare, by allowing greater involvement and feedback in the care process.

The gradual move to EHR is also pressed by other reasons, such as the growing need for physical storage in the classical paradigm of paper record, the considerable delay in getting the information after it is needed and the increasing difficulty in finding that same information. Another real push in healthcare IT systems is the need for billing, as the first systems to be introduced are likely to be related to billing.

We have already discussed the relevant security issues in chapter 2, and we should keep those in mind when planning an EHR, an integrant part of HIS, as their security is only as good as the weakest link.

Some countries have already introduced EHR, and lessons from their experience are very important. Networked EHR are the most challenging ones, with the added fears of possible record aggregation. There are various challenges in EHR deployment, such as the need for patients full disclosure, the legal and ethical obligations, the costs involved, the readjustment needed in current workflows and the possible proprietary technology dependence, which should be avoided whenever possible.

The move for a PHR may well be a subsequent step or even deployed alongside the EHR, as it is certainly, when carefully designed, a great aid for patient empowerment. It is, after all, his information. Various private companies have developed PHR, free or fee based, and with or without collaboration with healthcare entities. Such approaches must be taken with great care, but they are definitely projects worth following. 


\section{The Citizen Card}

\section{Identification beyond reasonable doubt}

Portugal, like most European countries, has had for many years now a government issued national identification paper based card, other than the passport (countries like the USA, Canada, Australia or the United Kingdom do not have such identification) [156, 157]. This document is used as the identification credentials before public and private entities. It is, however, in the process of being replaced by a more secure, practical and functional electronic document, which will incorporate other (previously issued separately) government documents [158].

Finland, for instance, was a pioneer in the electronic ID concept. Such cards were firstly issued by this country in December 1999. They were, however, optional, and not this deployment was not very successful (75.000 units emitted until December 2005) [156].

The Portuguese Citizen Card (Cartão de Cidadão - CC) is a multiple identification document which includes an optic machine-readable zone (MRZ) and an Integrated circuit (IC). It is meant to substitute 5 identification documents: National Identity; Social Security; National Health Service; Tax Payer's; Electoral Card (ID for elections). It is a move to an easier relationship between the government and the citizen, although it does not, in accordance to the Portuguese constitutional law, cross-reference the data between the public organisms to which the documents refer. This is done with the purpose of disallowing aggregation of the data derived from its multiple identification numbers, other than the numbers themselves. Public organisms also don't have the right to unilaterally decide to cross-reference these data.

In Figure 7 and Figure 8, the security aspects of the card are represented [159]. Table 1 enumerates the CC's information content and the place where it is held. 


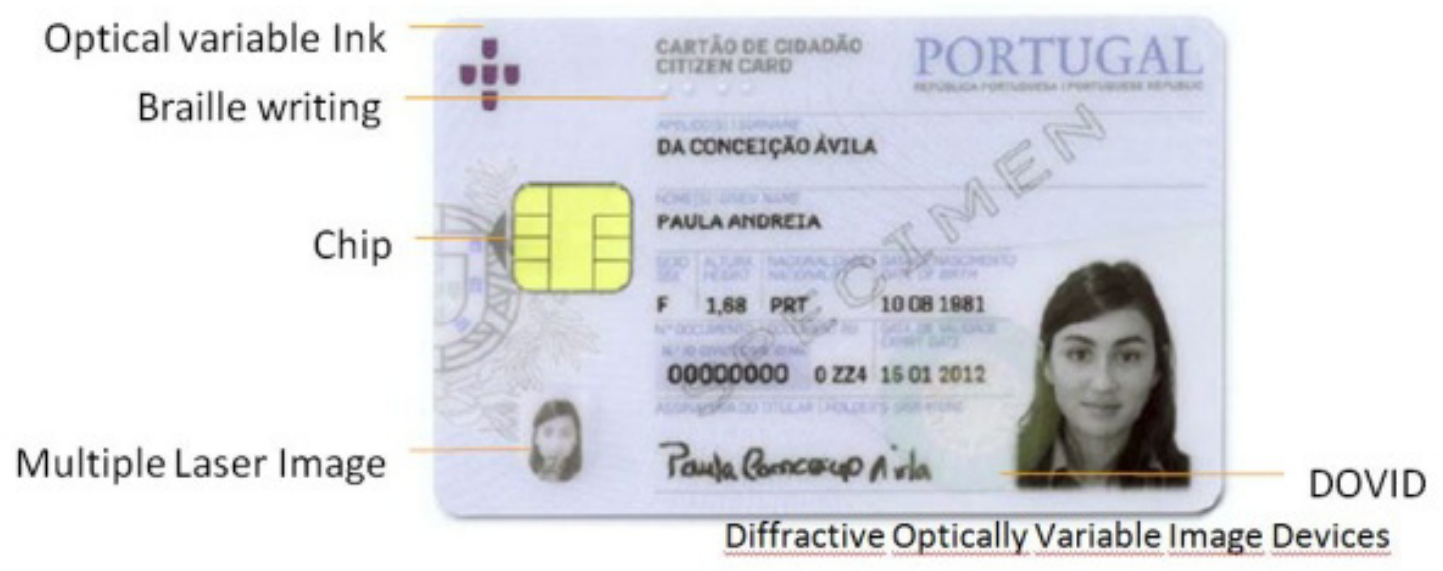

Figure 7: Security aspects of the CC front

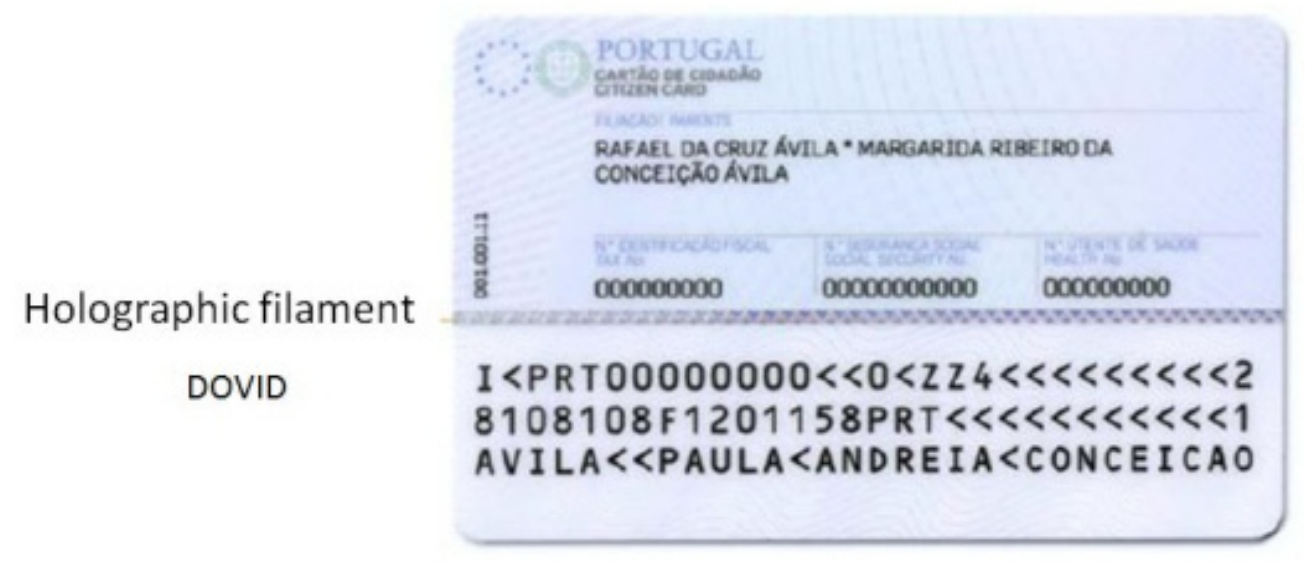

Figure 8: Security aspects of the CC back 
Table 1: CC identification elements

\begin{tabular}{|c|c|c|c|}
\hline Properties & Vis & MRZ & IC \\
\hline Last names & $x$ & $X$ & $X$ \\
\hline First names & $x$ & $x$ & $x$ \\
\hline Parents Names & $x$ & & $x$ \\
\hline Nationality & $X$ & $X$ & $X$ \\
\hline Birth date & $x$ & $x$ & $x$ \\
\hline Sex & $x$ & $x$ & $x$ \\
\hline Height & $x$ & & $x$ \\
\hline Facial Image & $x$ & & $\mathrm{X}$ \\
\hline Signature & $x$ & & \\
\hline Civil ID Number & $x$ & & $x$ \\
\hline Tax ID Number & $x$ & & $x$ \\
\hline Health ID Number & $x$ & & $x$ \\
\hline Social Security ID Number & $x$ & & $\mathrm{X}$ \\
\hline Document Number & $x$ & $x$ & $x$ \\
\hline Emitting Country (Portuguese Republic) & & $x$ & \\
\hline Type of document & & $X$ & \\
\hline Expiry date & & $X$ & $X$ \\
\hline Emission date & & & $X$ \\
\hline Address & & & $X^{*}$ \\
\hline Fingerprints (2) & & & $x+$ \\
\hline Eventual indications, according to the law & & & $X$ \\
\hline Authentication certificate & & & $x+$ \\
\hline Electronic signature certificate & & & $x+$ \\
\hline Software applications needed & & & $X$ \\
\hline Free writing zone for citizen use & & & $\mathrm{X}$ \\
\hline Additional health data (health sub-system, etc) & & & $\mathrm{X}$ \\
\hline
\end{tabular}

Vis: visible; MRZ: Machine Readable Zone; IC: Integrated Circuit; *Data not accessible; +PIN (password) protected access/use

The document number, which is the civil ID number plus 2 alphanumeric characters and 1 control digit allows to know the status of the card, namely if it has been revoked. 
This is a smart card capable of cryptographic operations, which is issued with two public key pairs. One pair is used for secure authentication and the other for digital signatures. These operations are aided by specific circuitry and software in the card:

- IAS (Identification, Authentication and Electronic Signature) - On-chip authentication and digital signing, which means the private keys never leave the card. The middleware permits direct communication with this application. It allows the signing of documents and authentication through a web browser;

- EMV-CAP - Random one-time password generation, for use in alternative channels, such as telephone;

- Match-on-card - fingerprint verification (only for use by official authorities in special circumstances)

The authentication certificate is activated when the card is delivered. The electronic signature certificate is activated only if asked for, and only by fully capable citizens over 16 years old. These certificates are revocable at all times, but their replacement is dependent on the issuing of a new document, because the private keys are unique copies, secretly stored inside the chip, and cannot be updated or retrieved

The card is also digitally signed by the emitting entity.

An application is available for download which permits user interaction with the aforementioned capabilities, as well as a middleware technical manual [160] to ease the integration of digital functionalities by public and private parties. This middleware is currently supported in the following environments (only latest versions listed):

- Microsoft Windows XP/Vista/7; Microsoft Windows Server 2003; Microsoft Windows 2000 SP3;

- $\quad$ Red Hat (linux) 5.3;

- OpenSuse (linux) 11;

- Ubuntu (linux) 9.1;

- Caixa Mágica (linux) 14;

- Mandriva (linux) 2008;

- Fedora (linux) 12;

- Apple MAC (Leopard/Tiger)

The card itself is an ID-1 format (ISO/IEC 7810), format TD-1 of machine readable travel documents (MRTD). The chip conforms to the position standard ISO 7816-2. The support 
material is polycarbonate. The Chip is currently an Infineon, model SLE66CX680PE, and the Operating system is Axalto ICitizen V2 64K (Table 2).

The chip complies with CC EAL5+, which is an evaluation built on the Smart Card IC Platform Protection Profile (BSI-PP-0002) [161]. This Protection Profile is endorsed by Eurosmart and complies with the internationally accepted standard ISO/IEC 15408 Common Criteria (see 3.3.1 - Evaluation/Certification).

Table 2: Operating system and chip characteristics

\section{Operating System characteristics}

Axalto, ICitizen V2 64K

Java Card 2.2.1

Global Platform 2.1

$64 \mathrm{~KB}$ of memory for application and data

$$
\mathrm{T}=0 \text { e } \mathrm{T}=1
$$

Multiple PIN Management

Dynamic Memory Management

\section{Chip characteristics}

\section{Infineon, model SLE66CX680PE}

$0.22 \mu \mathrm{m}$ technology, 16-bit processor 244 KB ROM, 6144 bytes RAM, 68 KB EEPROM

RSA 1024, 2048 bits /Eliptic Curve

DES, TDES (dual key), AES

CC EAL5+

Supply voltage range: $1.62 \mathrm{~V}, 3.0 \mathrm{~V}, 5.0 \mathrm{~V}$

Operating Temperature range: -25 to $+85^{\circ} \mathrm{C}$

500,000 write/erase cycles per page

Typical data retention 10 years

SAB 8051 compatible instruction set and some additional powerful instructions needed for smart card applications

Advanced security sensors and physical countermeasures (e.g. shielding)

True random number generation (AIS31 compliant)

Control of access rights to the memory by the memory management unit

Automatic error detection/correction of the NVM content data encryption for all CPU external memories by the integrated Memory Encryption and Decryption

Encryption of the data transported over the bus to and from the security sensitive SFRs Countermeasures against SPA, DPA, EMA, and DFA attacks 
The card certificates PKI is managed by the State Electronic Certification Authority (Entidade de Certificação Electrónica do Estado - ECEE). The key pairs are RSA 1024 bits [162]. They comply with European regulations on electronic signing and internationally accepted standards [20] (see Table 3).

Table 3: Standards implemented by the Citizen Card PKI

\section{Standards implemented by the Citizen Card PKI}

RFC 3647: Internet X.509 PKI - Certificate Declaration Practice and Certificate policies

RFC 2459: Internet X.509 PKI - Certification Revocation List and Certificate Profiles

RFC 3039: Internet X.509 PKI - Qualified Certificate Profile

RFC 2560: Internet X.509 PKI - Online Certificate Status Protocol - OCSP

ETSI TS 101456 - Policy requirements for certification authorities issuing qualified certificates

ETSI TS 101862 - Certificate Profile for Certificates Issued to Natural Persons

ETSI TS 102042 - Policy requirements for certification authorities issuing public key certificates

ISO 17799 - Code of practice for information security management/specification for information security management

The CC has a five years expiration date. It will be issued only to Portuguese citizens (with Portuguese nationality) and to Brazilian citizens covered by the "Porto Seguro" treaty. The latter documents will not serve as a travel document. The CC is not mandatory to whoever has a valid document substituted by it, but will be issued automatically whenever a change or renewal of one of the documents is requested.

The card states its version (physical and certificates) in the back, so that multiple and enhanced versions of the documents may be easily identified. There are three circulating versions of the document, with modifications related to the MRZ, to enhance its use and comply with ICAO (International Civil Aviation Organization) requirements. The first two MRZ versions may not be readable automatically [163].

The security is state of the art, but the possible security exploits are also perfected, and new ones can be found. There are various examples of smart card exploits, that companies try to circumvent and render unusable $[164,165]$. 


\title{
5.1 The Citizen Card Potential Role in Health Information Systems
}

\author{
Confidentiality, Integrity, Availability
}

The concerns of privacy and security in the medical informatics field are related to the need of professionals and patients to trust the system. IT security can only present the means for that trust to happen, in a non intrusive, easier way. Smart cards indeed offer an easy and practical way for the average person to become truly involved in a secure IT environment.

The task of expanding HIS outside home institutions, for a regional or national EHR, and for the creation of a Personal Health Record (PHR) which should interchange data with, or be based on the EHR [139], is very challenging, when it comes to security and trust management. A PKI with smart cards can be an effective solution for this problem. However, the deployment of such a solution can be very expensive and difficult [166]. The CC, being already in place, presents itself as a relatively inexpensive alternative and opportunity for securing HIS.

\subsubsection{Standard Healthcare Use of the Citizen Card}

The CC holds key data for healthcare provisioning, as shown in Table 1, without including health information, i.e., it holds only the minimal necessary identity information for admission in a hospital or other healthcare facility.

A legal authorization was issued in 2005 by the Portuguese National Data Protection Committee (CNPD) [167] for the utilization of data from the CC database by hospitals. This consultation of the database (which contains many of the CC data) may be done directly, by query trough the Rede de Informação em Saúde (RIS), upon authentication via login/password by the hospital.

During its proof of concept trials [168], the CC was analyzed for the purpose of electronic medical prescription. By connecting to a central database, and after authentication by the patient, a doctor can see the database and prescribe electronically. The prescription is stored (in real-time) for later viewing in the pharmacy, where, after the same authentication procedure, the pharmacist can see the prescription and provide the medication. The advantages publicized are the rationalizing of prescription (the application possesses a decision support system), fraud and error preventing, and the creation of a centralized prescriptions database. Another work on this subject presented similar solutions [169], and other countries are actively working on making e-prescribing a reality (see: Smart Cards Use in Healthcare). 
Recently, a national healthcare database (RNU - Registo Nacional do Utente) was made available to CC users, who may consult their administrative data online (such as health subsystem or primary care facility), upon authentication with the CC. The waiting list for surgeries is also available to consult and, shortly, appointments can be made directly (online) to the primary care physician).

\subsubsection{The Citizen Card in HIS security}

The CC is based on the latest, and well tested, smart card technologies. Its IC has top security and versatility features. As expected for such an important document, it supports state of the art active defenses against tampering, the latest encryption technologies and proven software from a major supplier, as well as good security certification. It has great support for the user, simple software, multi-platform compatibility, good technical guides, and the only thing the user has to aquire to use it online is a relatively inexpensive card-reader, which is available at low prices at CC stores (around $12 €$ ).

The CC's public key cryptography, based on RSA 1024 bit keys, is considered adequate for these purposes, although more secure keys (2048 bit) would ease the minds of the less optimistic, since the former is, nowadays, the minimum recommended by many authorities in this matter [170]. RSA is, however, a proven viable solution, and it makes good sense to adopt it for this purpose. The fact that two key pairs (authentication and digital signing) are being employed by the CC leads us to another line of security policy. The user needs to understand the difference between signing and authenticating (because it has to choose the operation), one can leave signing deactivated (e.g. for minors) and the compromise of a key does not compromise the other. The card also has a five years expiration date, which leads to a fairly short life cycle, so that card vulnerabilities can be addressed or security and cryptographic upgrades deployed in future revisions.

Overall, in the light of the current literature, the CC has the potential to be a good example for the deployment of these devices. Time will tell if the framework already in place will hold this expectation.

When it comes to Healthcare, the use of the $\mathrm{CC}$ solely for electronic prescription lacks ambition. This card has a much wider potential usage. Its strong authentication capability can be used for online banking, as well as public services identification. And it is already prepared to be used in this way by public and private entities, at the user's discretion. The healthcare system may greatly benefit from the CC. It provides static identifiers that can be used for 
preventing record duplication, or to find different institution records for the same person. It is unifying, as it can potentially be used to authorize a procedure in a hospital, fill a prescription in the pharmacy or access digital medical records from a doctor's private practice. It can be part of the answer to fears of privacy loss or security within the HIS, since by its use all actions could be logged and traced to a single person. It could also substitute many of the technologies being rolled out, which are more expensive (like biometrics), and lack the CC's readiness and deployment abstraction.

One of the main challenges has been the complexity involved in implementing an eHealth system and protecting the integrity and confidentiality of the communicated information. There are major IT infrastructure and paradigm changes that are often required to implement a new system [73].

Security implementations should overcome the differences in security architectures, giving each individual system autonomy for its own security administration, while complying with certain policies on data manipulation and transfer [37]. The user authentication can be made by presentation of credentials, which in this case could be the $\mathrm{CC}$, in any given healthcare provider, and transaction of data from other locations would be easily done, since the user/patient is authenticated and can authorize it.

\subsubsection{The Citizen Card as an Expansion Adjuvant of HIS}

One of the major problems encountered when planning for a regional/national health record is the lack of security features that would assure the privacy and confidentiality of such data. Health data is obviously something we would like to share, but only in special circumstances, and to very restrict group of authenticated people.

The difficulty in implementing secure web based access is related to the need of a strong authentication scheme, incompatible (in a practical sense) with the common password use and all its known risks, especially considering the often little knowledge and interest on IT security on the patient and healthcare professional side.

A Public Key Infrastructure helps to mitigate many problems pertaining to data security and accessibility. It is, unfortunately, difficult to implement, test and maintain. In such a system, every doctor wanting to use the patient's health record would have to be enrolled into the system in order to possess a card, and every patient would have to do the same. It is difficult to deploy such a wide system, not to mention the high costs involved. 
The advent of the national electronic identification card in Portugal, with the introduction of the Citizen Card, potentially solves most of these problems, as the card integrates RSA digital signature and authentication Keys, and will gradually replace current ID for all Portuguese citizens.

A web based framework with a central repository for information could be a good solution for a nationwide EHR, in which all players could have access to software in the form of continuously updated applets, thus needing only a browser and internet connection to be able to use the system. One of the most difficult aspects, in this scenario, is to warrant the security of the system. A PKI with smart cards is one of the best proven ways of doing so [171]. With the CC and a card reader, along with clearly defined security policies, this could no longer represent an issue, and instead be a source of great potential.

Secure remote authentication with this kind of framework leads to new possibilities, such as:

- A PHR managed, or co-managed, by the patient. This puts the patient in charge of their health records, giving him the opportunity to gather and distribute their health information as they see fit. This applies to collaboration with private companies, such the ones referred in 4.5.2 -The concept of Personal Health Record.

- Authorization of clinical data access in any place by the patient himself;

- Telemedicine or e-health: remote health work or remote expert consultation is a rapidly developing field. The $\mathrm{CC}$ could be used to secure connections between hospitals, to sign documents and reports with legal validity, making these applications less vulnerable and permitting an efficient allocation of personnel without harm for patients (e.g. Radiology experts).

- A true National EHR is very difficult to implement, but trust is the basis of such work. Trust from patients, from healthcare personnel, official entities and healthcare management. The $\mathrm{CC}$ has the potential of helping to create this trust, the same way people trust a bank. The $\mathrm{CC}$ is built to be secure, and its legal binding should facilitate various remote processes.

Of course, for most of these uses, much work has to be done other than the simple use of the CC authentication capabilities. Still, a part of the various expected problems can be more easily managed. 
One particular characteristic of the Portuguese National Health Service is that public healthcare is the standard, which could greatly facilitate deployment of such an architecture, as there are much less companies and entities to hinder the agreement on standards and policies.

As written in section 4.5.2 - The concept of Personal Health Record, there are a few companies involved in the maintenance of an online PHR as a paid service $[120,139]$. Recently, two major software companies (Google and Microsoft) joined the market with free PHR products $[151,153]$.

These rather new alternatives open some public/private collaboration opportunities, as they are already collaborating with various healthcare (namely private) entities. The patient has full control of their records. As some may feel uncomfortable trusting their health information to a private company, encryption could ease this feeling, or, at least, handling of access authorization via $\mathrm{CC}$, as a much more secure authentication method than $\operatorname{login} /$ password.

There are, however, a few aspects that are to be considered in such a move, and others that could undermine its use in HIS. Both aspects are addressed in the following sections.

\subsubsection{The Citizen Card and ISO 27799}

ISO standard 27799 [172] provides guidance to protection of health information by implementing ISO/IEC27002 [173], which has a more broad scope, for IT security.

Concerning the scope of the present work, there are a few items worth noting in ISO 27799:

\section{Access control}

As major orientations, organizations that deal with personal health information need to control it, in a way that precludes all use unless:

- The user has a healthcare relationship with the data subject

- The user is carrying out an activity for the data subject

- There is a need for specific data to support this activity

RBAC (Role Based Access Control) is recommended for the aforementioned control, along with Workgroup Based Access Control (clinical teams) and discretionary access control. 
It is noted that time pressures found in health delivery situations can make effective use of passwords difficult to employ, as well as the care needed when using wireless networks, especially regarding the use of encryption standards that are largely ineffective, such as Wireless Equivalent Privacy (WEP).

Regarding Teleworking/Telemedicine, the legal implications of one being able to work out of borders or even out of jurisdiction (e.g. international waters) have to be taken into account. It is necessary to uniquely identify the subjects of care and there is a necessity for audit trails, logs and other evidence on security incidents, as well as plans to quickly respond to such incidents.

As noted in the previous sections, the $\mathrm{CC}$ can be an effective instrument to overcome and exceed the orientations on this matter. Authentication may be facilitated, and is greatly improved over other methods, namely login/password. Teleworking, or Telemedicine, is substantially easier because of the legal binding, secure authentication and enhanced privacy that the CC functionally permits. Unique patient identification can also be accomplished by the use of the CC SNS number. Audit trails are greatly facilitated and/or improved because of the secure identification of users, time and place, as well as the framework for digital signing and consequent respect and confidence in the system and the electronic documents it contains.

\section{Threats to HIS security and the role that can be played by the CC}

As threats to health information security, ISO 27799 states some examples. The CC may be used to help address many of them:

- Masquerade by insiders - The use of legitimate accounts by those that do not own them. The complexity of this threat is that this is sometimes done because it makes work easier, such as continuing work in a workstation logged on by someone else. It is a serious and very common security breach.

- While some vulnerabilities may persist, use of the CC would make this more difficult, because logging may be dependent on the card insertion on the machine, and with few additional steps (automatic and/or timed logout, impossibility of concurrent logins, etc) a good level of protection would be assured

- Masquerade by service providers - In which someone has a legitimate reason to use the system, such as maintenance or testing, but uses his credentials to gain unauthorized access to data. 
- Depending on the situation, CC login would make the transactions and access, at least, traceable, if logs are implemented and available. Some kinds of low level access are, tough, difficult to control.

- Masquerade by Outsiders - when an unauthorized $3^{\text {rd }}$ party gains access to data and/or systems by impersonation of a legitimate user, or fraudulently becoming a authorized user (social engineering). This may constitute a fail in one or more security controls, such as

$\bigcirc$ User identification

○ User authentication

$\bigcirc$ Origin Authentication

$\circ$ Access control and privilege management

- The CC can overcome most of these shortcomings, by ensuring secure authentication of people. With proper policies, access and privileges may be easily controlled.

- Unauthorized use of a health information application- Either an unauthorized user in an unattended workstation, for example, or an authorized user performing unauthorized actions, such as maliciously altering data. This may mean a failure of one or more controls:

- Workgroup access control - by permitting access when the there is no relationship to the subject

- Logging and audit control - failure to notice actions

- Personnel security - inadequate training of personnel

- By contributing for an easy to use and reliable RBAC, or other privilege management system, along with proper logging, actions could be monitored and audited.

- Malware - damaging or disruptive software that may harm, hinder or disrupt the HIS. This may be a virus or a worm, and is potentially very dangerous, if it manages to bypass security software.

- The use of card readers with PIN pad, while not solving this problem (which has specific solutions), enhances the security of the framework by denying malware, such as key loggers, access to the PIN associated with the cards. 
- Misuse of System resources- Non work related use of systems, especially when potentially compromising.

- The problem may be overcome by informing the personnel on the security risks involved in some actions. However, it may be discouraging to do forbidden actions when the identity is known and logged, by use of the CC.

- Communications Infiltration - Tampering with the normal flow of data by an individual. This includes Denial of Service (DoS) attacks, and relates mainly to system architecture, vulnerability assessment and (lack of) intrusion detection.

- DoS attacks are harder to perpetrate when a token, such as the $\mathrm{CC}$, is required for access to a network.

- Communications interception - lack of encryption in communication between machines leads to several problems, such as interception of data. Packet sniffing (which is the interception and reading of network packets free flowing through the local area network (LAN), for example). This way, unencrypted emails sent over this network can be readily intercepted and read.

- Securing the network with encryption based on VPNs, where the client can be authenticated by the CC is an easy way to overcome this security threat.

- Repudiation - This threat to security relates to the deniability of an action, such as sending or receiving a message. It implies failure to ensure adequate control on the work flow.

- The CC holds hardware and software for secure authentication and electronic signature. Thus repudiation is, at least, as difficult as with a signed paper. As pointed in this paper, proper use of these signatures may be much more secure and versatile than written signatures.

- Connection failure - Network service disruptions or server "down time" (not accessible: by failure, maintenance, etc).

- These disruptions may lead to diverting traffic through the internet, for example, in a less secure way. The CC allows for easy implementation of measures to ensure proper and secure transmission of data trough an unsecure channel. Network disruptions are, still, an important problem in IT security, which requires careful planning of the infrastructure and vital systems, with redundancy of drives, machines, power supply and network systems.

- Embedding of malicious code - use of malicious code disguised in legitimate pieces of data, such as email. 
- Mail electronic signing, for example, constitutes another layer of security against fraudulent emails, and is easily set up by the CC.

- Accidental misrouting - Sending data to wrong or fake addresses.

- Correct authentication of people and encryption of communications may be aided by having a proper set of certificates to trust. The CC PKI allows for correct identification of both sides involved in communication.

- Technical failure of the host, storage facility or network infrastructure - these failures constitute very important threats, sometimes critical, are many times related to improper planning of the HIS.

- Environmental support failure - power failures, natural or man-made disasters may lead to failure of HIS, in times when they are needed. These have to be built considering those scenarios.

- System or network software failure - leads to weaknesses in areas controlled by the software.

- By adding another layer of security, by denying access to the network without the $C C$, these exploits may be less successful.

- Application software failure - Improper designing of software may facilitate exploits, utilized to disrupt (e.g. DoS attacks) or compromise (e.g. allowing disclosure of information).

- The CC adds another layer of security in accessing the application.

- Operator/user error - unintentional disclosures, loss of data or other errors.

- While not preventing it, the CC allows for correct identification of the person/people involved, so that proper measures can be applied.

- Maintenance error - lack of proper maintenance of systems.

- The CC may contribute to correct logging of programmed maintenance, ensuring compliance with the maintenance schedule, as well as identification of personnel involved in errors.

- Staff shortage - implies little manpower to accomplish all necessary actions. In healthcare, epidemics may simultaneously add to system load and cut on manpower availability.

- The system automation in areas such as disaster recovery and "break the Glass" features (using feature or performing a restricted action in emergent situations) along with proper logging and the CC may allow for better uptime 
with less manpower, while maintaining adequate and possibly improved levels of security.

- Theft and willful damage - Performed by insiders or outsiders, may constitute important security breaches in HIS.

- While not preventing these actions, the CC may make them harder to accomplish, by using access controlled areas to store critical hardware, and logging access to these areas.

- Terrorism - Has to be considered in the planning of HIS.

- The CC, being useful in the areas described above, may reduce the chance of a successful outside attack on HIS.

\subsubsection{Anticipated Problems with the Use of CC in a HIS}

\section{Problems and solutions}

The spread of innovation in a healthcare environment can be impaired by some issues:

- There is the need to enroll the help of various medical and paramedical professionals, with different backgrounds, especially regarding the use of new technologies.

- There is the need to involve the patient, as the client, especially in the EHR setting.

The inertia on processes, defined as the impediment caused by rooted practices or lack of stimuli to change, by need or obligation, is difficult to overcome. There is therefore a need to find ways to do so: Identifying the problem to which it is related, in order to ascertain plausible solutions.

\section{Professional's potential problems in the usage of the CC}

Will the use of an identification card in a professional setting be easily accepted by healthcare personnel? Is the smart card a usable and useful method for digital signature and authentication on the workplace?

Healthcare professionals have special ways of working. They do a lot of multi-tasking, tend to have busy times with various issues on their minds, and often deal with delicate situations and even life or death decisions. Such a stressful and busy line of work is not always compatible with the IT requirements or work flows, and that is one of the reasons HIS do sometimes fail to improve care. IT tends to be organized and rigid, far from the apparent chaos involved in diagnosing a disease, for example [174, 175]. 
This way, and although the use of CC could substantially improve the healthcare professional relation with the HIS, it should be stressed that it has to be on a clearly helpful, understandable and unobtrusive way. Like any other setting, the system should be tested for usability. There may be some resistance in using one's ID card in the line of work, every time interaction with the system is needed, it may take longer than ordinarily takes to log. There is always the possibility of forgetting the token, which would undermine the system. There is also the obvious problem of non Portuguese healthcare workers, which cannot have a similar card, though a quite simple solution would be to have a small setting PKI, and issuing cards for those workers. Solutions like the one's adopted by France or Germany, with dedicated cards, would obviate this problem, while lacking most of the aforementioned advantages.

An acceptance testing took place in Slovenia regarding smart cards in healthcare, and results were positive, but those were healthcare dedicated cards, not national identity ones [88].

The compatibility of Health Information Systems with healthcare workflow has been thoroughly debated, and it is not easy to find proof of positive and effective contribution to healthcare [129]. Many systems have failed because of little integration in everyday tasks, cumbersome use and deviation of what professionals consider important for the accomplishment of their daily work[176].

It is difficult, or even impossible, to establish rigid workflows in healthcare. Many of the tasks done obey rigid schedule and order, but exceptions are far too common to be worthy of such designation. The process of diagnosis, for example, varies widely among doctors, because of the mechanisms involved in such a complex process $[174,175]$. The concept of multitasking gains new perspective within an infirmary with tens of patients, and the priorities are many times defined "on the fly" because of unforeseen events, forcing the rearranging of the workflow[177].

When one tries to adapt humans to the requirements of a machine, the result is less than optimal, unless people are actually working inadequately and their tasks are not productive, in which case the optimization of the tasks leads to improvement, not necessarily because of the new system. The cautious approach is to understand the workflows and to what degree they vary, the aspects that need improvement, whether because of the rule of law, internal policies, or simply because they are deficient, and to observe ways to facilitate tasks that are troublesome or boring, for example. 
One should not expect doctors and nurses to alleviate accounting work by registering meticulously treatments administered, procedures done, materials spent, diagnosis made, etc, if the additional work involved in that process is not easy and/or compensated, as additional time in front of the computer obviates time at the patient's bedside, and corresponds to administrative work not easily understood or valued to their line of duty. It isn't easy to accomplish tasks that will not bring benefits for the staff or their patients [178].

Using a smart card for logging in the HIS solves a lot of issues in system security and patient privacy, but that does not necessarily brings practical advantages to the user. The most used authentication method is login/password, insecure by nature, and sometimes difficult to use when more secure passwords are employed (alphanumeric with special characters, long passwords, changed periodically, etc) [7]. From a user perspective, on the other hand, login/password is not hard to use, if one chooses a simple, easy to remember password, its free and has been used for decades in IT [6]. So, when changing this simple, though many times wrongfully used, authentication method, a correct approach may be to make login/logout even simpler (such as smart card automatic login) and/or to make workers aware of the dangers (to itself and patients) of password authentication, in order to smooth the initial adaptation time:

- A machine will allow interactivity only when a smart card is inserted. The smart card is authenticated centrally by means of certificate verification, so a role is attributed to the user, as well as all the permissions issued to that role. All interaction with the healthcare software will be permitted based on the role and automatically logged upon launching. If the smart card is removed from the station it logs off automatically. Further electronic signing of documents created is prompted to the user, and the introduction of the correspondent PIN substitutes the handwritten signature.

There are some possible problems with this theoretical approach:

- If a doctor or nurse needs to be constantly leaving a certain space in order to see/treat patients, then returning to the station to register its findings or procedures, this login process can be bothersome, especially if accompanied every time by password introduction, and possibly the time need to reach the page they were working previously, even if by some clicks.

- There is the possibility of losing the smart card, either by forgetting it in a station or in the clothing, a problem aggravated if the card serves even higher purposes (Identification, legal signing...). 
- If someone lefts the wallet at home, it would be essential to have an alternate method of authentication, permitting use of the system. Alternatives could be: Password authentication, properly enforced and justified for a period of time (e.g. a workday); a card issued by a supervisor/superior, that logs in the system as the real user, but with the remark of temporary ID, with logging of user, issuer and reusable card used.

These problems could be reduced if the $\mathrm{CC}$ had a contactless chip, in which case the professional would simply have to pass it close to the reader or stand next to it.

On the other hand, if such use for the $\mathrm{CC}$ is accepted, it may have a positive general effect in the careful application of security measures, because:

- It is an important item in everyday life, one that people will not want to share or loose, such as a credit card.

- The legal binding and non repudiation of actions taken enforces correct use of systems, with less exposure to possible harm (e.g. internet sites, malicious files), as well as potentially increasing the quality of records.

The end user will also be keener to use a smart card if:

- A perceived benefit, such as simplification of a task or process, is realized.

- The possibility of greater interaction with central services online, even from home, as well as access to statistics tools, for example, would make staff aware of a direct benefit of the system. The idea of a safer and more private network must be passed on, but with a broader explanation of the differences between now and before, as most healthcare workers won't realize them.

- The innovation is perceived as superior to the technology it is replacing; there is social prestige or other attributes linked to the use of the innovation [75].

- Its use is convenient, i.e., user-friendly and not very different from previous routine

- Everyday use should be equivalent or simpler than the previous authentication system. If possible, it should be also easier to use.

- There is integration with the practices, values and culture of personnel [75].

- The system is trustworthy, and transparent. The mechanisms involved in the smart card use are understood by users, without hidden functions. 
- There are alternatives to smart card use, so people have time to adapt and adjust, as well as perceiving the real benefits of adoption

- As an alternative, password authentication could still be used, but with security enforcing on the password chosen or other security token like a bank card. Such a dual authentication should be on test until every aspect of the smart card use could be understood.

\section{Patient's potential problems}

Less literate patients may have problems with using the card (and be potential targets for fraud), although CC use is similar to the use of a common debit card, quite common for many years now. There are also special requirements, particular to healthcare, that need to be addressed, such as ways to access information in an emergency situation, when the citizen does not have its card or is not conscious.

As with the healthcare workers, not all patients will be able to own a CC, only Portuguese citizens and Brazilians covered by the Porto Seguro treaty.

Following advices from previous smart card implementations, it is necessary to have and demonstrate clear advantages for the use of the card, as well as a marketing support and campaigns to support them [75]. In this sense, optimal information on the need for excellent health records for excellence healthcare, and the active role of the patient in providing and aggregating this information, vital to himself, must be passed on to the citizen/client/patient.

Patients should understand the importance of the security of their information, and the role that the CC may have on it, so they may understand and support its use under these conditions. Furthermore, implementation must follow according to their values and culture [75].

\subsection{Utilization Scenarios}

As a brief summary of what can be accomplished with the CC in healthcare, given all the information already covered, a few scenarios can be depicted. Apart for some minor software implementations and adaptations, a HIS already in place with minimal security can be easily adapted for use with the CC. A smart card reader, which is fairly cheap and durable, is needed for each workstation. 


\subsubsection{Authentication}

Authentication in HIS may be done by token alone, or token plus password, in this case the CC. This may be a solution for some problems described earlier in which it may be laborious to insert the PIN number many times over a short period of time. However, this is a compromise decision that can be adopted in certain secure settings, such as hospital workstations in restricted areas, in order to minimize the impact from logon process, or the circulation of forged tokens (which can become theoretically possible without enforcing the need for authentication PIN).

In other settings, authentication may be done via authentication PIN, and this way, over a secure connection (HTTPS, for instance) a hospital worker can login from his home or other location with a computer. This method allows for much greater security than the simple insertion of a card in the reader.

- In an emergency a neuroradiology expert, who is off the clock, is contacted to receive and report on an exam which can alter the course of treatment of a car crash victim.

- A small hospital can contact an expert oncologist from another institution for advice on a rare disease and ask him to login and review the EHR from the patient.

- Experts highly trained in small fields can work in central hospitals and give support to smaller ones, which wouldn't need such individuals at full time. Patients wouldn't have to be transferred so often by lack of expert advice.

- Patients could look into their PHR and give access to their chosen doctors (private or other), outside the institution which collected the data.

\subsubsection{Digital Signing}

Nowadays most important documents are printed, or handwritten, and hand signed. With the $\mathrm{CC}$, all reports and documents could be electronically signed, so that all requisites for Integrity and non repudiation are covered. The digital signature present in CC, as already discussed, is a "qualified signature" and attains the highest level considered for signing documents (for citizens) being legally equal to the graphic handwritten signature on paper $[158,179]$. Digital signing involves a specific action of the user, so it is not mistaken with authentication.

- Reports can be instantly available, without communication of results by insecure channels, such as email or telephone, because a digital signed paper is ready to be 
viewed, downloaded, or sent, and there is certainty of the creator of the document, as well as the time of creation.

- Patients can do the same for various purposes, such as informed consent.

- Paper would not be needed for legal binding reports or documents, and availability of such documents would be theoretically much better.

- Signatures can be verified within the CC PKI.

\subsubsection{Emergencies}

Although a secure access to data is always better for the safety and privacy of the data, it limits availability in some extent. One may need information in an emergency and the usual means to get it may be unavailable, such as in a emergency setting in which a car crash victim is unconscious, and his EHR is blocked, be it because the victim isn't from that area (no records in the hospital) or because the EHR, by principle, cannot be accessed without his explicit consent.

- A "break the glass" feature may be implemented, in which some individuals may access data without authorization, and justify afterwards the need for the action. This is relatively easy in a well implemented access control based on the CC, because authorized accesses may be logged, and "break the glass access could produce a "flag" which is directed to the responsible for audit, who then analyses the situation.

- In a trip to another country, if medical care is needed, an EHR/PHR may be easily consulted via a HTTPS link in an ordinary browser. This may be allowed by default, or, to enhance security, only if the trip description is logged into the system. 


\section{Conclusion}

The deployment of the CC in a HIS environment, although less complex and expensive than starting from scratch, still demands much hard work for testing all the functionalities and potentialities described in this document.

Security is not exclusively dependent on authentication or on the user. This is just one of the (many) important items of IT security. It is, nevertheless, one of the most difficult to accomplish, because it directly concerns the end users and, especially in the HIS setting, relies on the perceived security of people, mostly not directly related to IT, many times even greatly unaware of IT risks, security and its implications.

Securing HIS is not an easy job, but it may become significantly easier with the implementation and integration of an architecture that supports the CC. Role Based Access Control, for example, could be easily built if such an infrastructure is already in place. Such move could also greatly improve patient empowerment and confidence, effectively enforcing the rights stated by the rule of law. Privacy could be enhanced despite the move to effectively more ubiquitous and transferable contents, because the $\mathrm{CC}$ would provide the tools necessary for secure transactions.

Compared to biometry, the CC offers multiple advantages, because the system implementation is generally cheaper (the hardware is much cheaper and cards are already issued), the CC is (in practice) irreproducible, whereas most biometry is widely available (we leave fingerprints and palm prints all the time, our gait can be filmed and the iris photographed - and various attacks on these authentications methods are known). The CC may be used to sign documents in a secure and widely accepted format and it may be revoked and reissued if lost, or stolen, whereas biometry cannot.

In a move to enhance HIS, rather than repair its current flaws, a much better EHR and PHR could be securely created, oriented for the better care of people, yet possibly producing great savings with very little financial costs. The grounds for expanding telemedicine, providing global and complete care out of the metropolis and the bigger hospitals, while maximizing manpower and equipment. In a country which assures an essentially equal and free health system for everyone, this translates in genuine savings for the tax payers, and possibly better allocation of financial resources. The $\mathrm{CC}$, by providing effective tools to clearly identify the origin of data, could also addresses concerns of intellectual property. 
HIS cannot, and should not, be allowed to grow and expand as long as their security is less than sate of the art. This is paramount because the real improvement of this move can be greatly impaired by problems, possibly catastrophic, related to security accidents, that can thwart the system by irreparably provoking damages (e.g. to privacy) and effectively undermining confidence in IT usage in healthcare. Networks of computers have been around for many years and much experience can be drawn from past mistakes, so these should not be repeated. More than upholding the law, HIS security must protect individuals the same way a trusting doctor is sworn to secrecy.

The CC is a major technological advance in a number of areas in Portugal, and it can be so in e-health, by helping secure existent HIS. It can also facilitate the broad acceptance of IT in healthcare.

Much more studying and testing is needed, as this can only be considered a first and very preliminary tackling of an old, but often overlooked problem. It would be nothing short of unfortunate to miss this opportunity to try to really enhance HIS without jeopardizing the privacy and security of these systems, while capitalizing on a great investment, already made and ready to be utilized. The players are there, and they play fine... We should just find a way to make them play together. 


\section{Bibliography}

1. Reichertz, P.L., Hospital information systems--Past, present, future. International Journal of Medical Informatics, 2006. 75(3-4): p. 282-299.

2. Haux, R., Health information systems - past, present, future. International Journal of Medical Informatics, 2006. 75(3-4): p. 268-281.

3. Oliver, A., Public-sector health-care reforms that work? A case study of the US Veterans Health Administration. The Lancet. 371(9619): p. 1211-1213.

4. Win, K.T., A review of security of electronic health records. HIM J, 2005. 34(1): p. 13-8.

5. Wimalasiri, J.S., P. Ray, and C.S. Wilson. Security of electronic health records based on Web services. in Enterprise networking and Computing in Healthcare Industry, 2005. HEALTHCOM 2005. Proceedings of 7th International Workshop on. 2005.

6. Morris, R. and K. Thompson, Password security: a case history. Communications of the ACM, 1979. 22(11): p. 594-597.

7. O'Gorman, L., Comparing passwords, tokens, and biometrics for user authentication. Proceedings of the IEEE, 2003. 91(12): p. 2021-2040.

8. Furnell, S.M., I. Papadopoulos, and P. Dowland, A long-term trial of alternative user authentication technologies. Information Management \& Computer Security, 2004. 12(2): p. 178-190.

9. Piper, F. and S. Murphy, Cryptography: A Very Short Introduction. Very Short Introductions. 2002, New York: Oxford University Press. 160.

10. Shannon, C.E., Communication Theory of Secrecy Systems. Syst. Tech. J., 1949. 28: p. 656-715.

11. National Institutes of Health. The Hippocratic Oath. 20091 June 2009 [cited 2009 September 1]; Available from: http://www.nlm.nih.gov/hmd/greek/greek_oath.html.

12. Wynia, M.K., et al., Medical Professionalism in Society. N Engl J Med, 1999. 341(21): p. 1612-1616.

13. Senate and House of Representatives of the United States of America, Health Insurance Portability and Accountability Act of 1996. 1996.

14. U.S. Department of Health and Human Services, Office for Civil Rights HIPAA Privacy Rule Summary. 2003.

15. Committee of Ministers to member states on the impact of information technologies on health care - the patient and internet, Directive 95/46/EC of the European Parliament and of the Council of 24 October 1995 on the protection of individuals with regard to the processing of personal data and on the free movement of such data. 1995: Official Journal of the European Communities. p. 19.

16. Assembleia da República, Lei 12/2005 Informação Genética Pessoal e de Saúde. 2005: Diário da Républica. p. 606-611.

17. Anderson, R., Security in Clinical Information Systems. 1996, University of Cambridge: Cambridge.

18. Comissão Nacional Protecção de Dados, Relatório de Auditoria ao Tratamento de Informação de Saúde nos Hospitais. 2004. 
19. Mandl, K.D., P. Szolovits, and I.S. Kohane, Public standards and patients' control: how to keep electronic medical records accessible but private. BMJ, 2001. 322(7281): p. 283-7.

20. European Parliament and the Council of the European Union, EU Directive 1999/93/EC, Community framework for electronic signatures, in Official Journal of the European Communities. 2000. p. 12-20.

21. Van Der Haak, M., et al., Data security and protection in cross-institutional electronic patient records. International Journal of Medical Informatics, 2003. 70(2-3): p. 117-130.

22. Diffie, W. and M. Hellman, New directions in cryptography. Information Theory, IEEE Transactions on, 1976. 22(6): p. 644-654.

23. Preneel, B., A survey of recent developments in cryptographic algorithms for smart cards. Computer Networks, 2007. 51(9): p. 2223-2233.

24. Robshaw, M.J.B., Stream Ciphers, in RSA Laboratories Technical Report TR-701. 1995, RSA Laboratories: Redwood City.

25. RSA Laboratories. What is a stream cipher? 2009 [cited 2009 September 1]; Available from: http://www.rsa.com/rsalabs/node.asp?id=2174.

26. National Institute of Standards and Technology. Computer Security Resource Center. Computer Security Division 2006 10/12/2008 [cited 2008 10/12/2008]; Available from: http://www.csrc.nist.gov/pki/HashWorkshop/NIST\%20Statement/Burr_Apr2006.html.

27. Diffie, W., The first ten years of public-key cryptography. Proceedings of the IEEE, 1988. 76(5): p. 560-577.

28. RSA Laboratories. The RSA Factoring Challenge. 20092009 [cited 2009 September 1]; Available from: http://www.rsa.com/rsalabs/node.asp?id=2092.

29. Pietiläinen, H., Elliptic curve cryptography on smart cards. 2000.

30. De Dormale, G.M., et al. Low-cost elliptic curve digital signature coprocessor for smart cards. in Proceedings of the International Conference on Application-Specific Systems, Architectures and Processors. 2006.

31. Neame, R., Communications and EHR: Authenticating who's who is vital. International Journal of Medical Informatics, 2000. 60(2): p. 185-190.

32. Xiao, Q. Security Issues in Biometric Authentication. in Proceedings of the 2005 IEEE. 2005.

33. Zorkadis, V. and P. Donos, On biometrics-based authentication and identification from a privacy-protection perspective- Deriving privacy-enhancing requirements. Information Management \& Computer Security, 2004. 12(1): p. 125-137.

34. Kent, J. Malaysia car thieves steal finger. 2005 32st March 2005 [cited 2009 September 1st]; Available from: http://news.bbc.co.uk/2/hi/asia-pacific/4396831.stm.

35. Alert Life Sciences Computing SA. Alert Homepage. 2009 [cited 2009 July 8]; Available from: http://www.alert-online.com/?msg=online\&idLang=1.

36. Allaert, F.A. and C. Quantin, Le dossier médical personnel du patient : réflexions sur le portail d'accès unique et le masquage du dossier. IRBM, 2009. 30(3): p. 114-118.

37. Blobel, B., Authorisation and access control for electronic health record systems. International Journal of Medical Informatics, 2004. 73(3): p. 251-257. 
38. Brandner, R., et al., Electronic Signature for Medical Documents - Integration and Evaluation of a Public Key Infrastructure in Hospitals. Methods of Information in Medicine, 2002(4): p. 321-330.

39. Sucurovic, S., Implementing security in a distributed web-based EHCR. International Journal of Medical Informatics, 2007. 76(5-6): p. 491-496.

40. Hovav, A. and Y.J. Kim. Determinants of IP Version 6 Adoption. in Computing in the Global Information Technology, 2006. ICCGI '06. International Multi-Conference on. 2006.

41. Stallings, W., IPv6: the new Internet protocol. Communications Magazine, IEEE, 1996. 34(7): p. 96-108.

42. Ferraiolo, D. and R. Kuhn, Role-Based Access Controls. Proceedings of the 15th National Computer Security Conference (1992), 1992: p. 554 - 563.

43. Sandhu, R.S., et al., Role-based access control models. Computer, 1996. 29(2): p. 38-47.

44. Kammer, R.G., Digital Signature Standard. 2000, US Department of Commerce/National Institute of Standards and Technology: Federal Information Processing Standards Publication.

45. Tulu, B., et al., Implementing digital signatures for healthcare enterprises: The case of online disability evaluation reports. International Journal of Healthcare Technology and Management, 2005. 6(4-6): p. 470-488.

46. ArchiSig project, Conclusive and Secure Long Term Archiving of Digitally Signed Documents. 2006.

47. Lekkas, D. and D. Gritzalis, Long-term verifiability of the electronic healthcare records' authenticity. International Journal of Medical Informatics, 2007. 76(5-6): p. 442-448.

48. Bertino, E., B. Carminati, and E. Ferrari, XML security. Information Security Technical Report, 2001. 6(2): p. 44-58.

49. Ekelhart, A., et al., XML security - A comparative literature review. Journal of Systems and Software, 2008. 81(10): p. 1715-1724.

50. Shelfer, K.M. and J.D. Procaccino, Smart Card Evolution. Communications of the ACM, 2002. 45(7): p. 83-88.

51. Lu, H.K., Network smart card review and analysis. Computer Networks, 2007. 51(9): p. 2234-2248.

52. International Organization for Standardization, ISO/IEC 7816-1: Identification cards -Integrated circuit(s) cards with contacts -- Part 1: Physical characteristics 1998: Geneve.

53. NFC Forum. Near field Communication. 20087 December 2008]; Available from: http://www.nfc-forum.org/home.

54. Sun Microsystems. Java Card Forum. 2008 [cited 2008 December 10 2008]; Available from: http://www.javacardforum.org/.

55. Sun Microsystems. About Java Technology. 2009 [cited 2009 Sept]; Available from: http://www.sun.com/java/about/.

56. Sun Microsystems. Sun Opens Java. 2009 [cited 2009 Sept]; Available from: http://web.archive.org/web/20080513023707/http://www.sun.com/2006-

1113/feature/story.jsp. 
57. Sun Microsystems. Java Card 3. 2009 [cited 2009 Sept]; Available from: http://java.sun.com/developer/technicalArticles/javacard/javacard3/.

58. Gemalto. Gemalto Achieves Major Breakthrough in Security Technology with Java Card(TM) Highest Level of Certification. 2007 [cited 2009 Sept]; Available from: http://www.gemalto.com/php/pr_view.php?id=239.

59. International Standardization Organization, IT-Identification cards-Integrated circuit, ISO 7816. 1989: Geneva.

60. International Standardization Organization, International standard ISO/IEC 14443. 2003.

61. Dhem, J.-F. and N. Feyt, Present and Future Smart Cards, Gemplus, Editor. 2001.

62. Renaudin, M., et al., High Security Smartcards, in Proceedings of the conference on Design, automation and test in Europe - Volume 1. 2004, IEEE Computer Society.

63. Garcia, F.D., et al. Dismantling MIFARE Classic. in Esorics 2008: 13th European Symposium on Research in Computer Security. 2008. Malaga, Spain: Springer.

64. Mifare crack to be published as legal fight fails. Card Technology Today, 2008. 20(7-8): p. 1, 3-1, 3.

65. Gans, G.D., J.H. Hoepman, and F.D. Garcia. A practical attack on the MIFARE Classic. 2008. London, ENGLAND: Springer-Verlag Berlin.

66. Common Criteria. Common Criteria. 2008 [cited 2008 December 9 2008]; Available from: www.commoncriteriaportal.org.

67. Common Criteria, Common Criteria for Information Technology Security Evaluation: Part 3: Security assurance components 2007.

68. Smart Card Alliance, HIPAA Compliance and Smart Cards: Solutions to Privacy and Security Requirements. 2003, Smart Card Alliance: Princeton Junction.

69. Chan, A.T.S., WWW+smart card: towards a mobile health care management system. International Journal of Medical Informatics, 2000. 57(2-3): p. 127-137.

70. Stead, W.W., Rethinking Electronic Health Records to Better Achieve Quality and Safety Goals. Annual Review of Medicine, 2007. 58: p. 35-47.

71. Frist, W.H., Shattuck Lecture: health care in the 21st century. N Engl J Med, 2005. 352(3): p. 267-72.

72. Dwivedi, A., et al. Towards a practical healthcare information security model for healthcare institutions. in Proceedings of the 4th Annual IEEE Conf on Information Technology Applications in Biomedicine, UK. 2003.

73. A smarter way to stay healthy. Card Technology Today, 2007. 19(2): p. 14-16.

74. Houdeau, D., European Citizen Card Combined with Travel Document Function, Convergence or Divergence?, in ISSE 2006 - Securing Electronic Busines Processes. 2006, Vieweg. p. 25-29.

75. Aubert, B.A. and G. Hamel, Adoption of smart cards in the medical sector: the Canadian experience. Social Science \& Medicine, 2001. 53(7): p. 879-894.

76. European Commission, eGovernment Factsheet - Denmark. 2008.

77. European Commission, eGovernment Factsheet - Finland. 2008. 
78. The Social Insurance Institution of Finland. The Kela card. 2008 [cited 200823 November]; http://www.kela.fi/in/internet/english.nsf/NOS/171203103605MH?opendocument.

79. Manaouil, C., Le dossier médical personnel (DMP) : " autopsie » d'un projet ambitieux ? Médecine \& Droit, 2009(94): p. 24-41.

80. Marschollek, M. and E. Demirbilek, Providing longitudinal health care information with the new German Health Card--a pilot system to track patient pathways. Computer Methods and Programs in Biomedicine, 2006. 81(3): p. 266-271.

81. Blobel, B. and P. Pharow, A model driven approach for the German health telematics architectural framework and security infrastructure. International Journal of Medical Informatics, 2007. 76(2-3): p. 169-175.

82. Bruce, S. Germany's electronic health card stalls. 2009 April 2009 [cited 2010 January 23]; Available from: http://www.ehealtheurope.net/news/4757/germany\%E2\%80\%99s_electronic_health_c ard_stalls.

83. Borchers, D. Loss of Data has Serious Consequences for German Electronic Health Card The H Security 2009 [cited 2009 August 9]; Available from: http://www.honline.com/security/Loss-of-data-has-serious-consequences-for-German-electronichealth-card--/news/113740.

84. Karl A. Stroetmann, T.J., Alexander Dobrev, Veli N. Stroetmann, eHealth is Worth it? The economic benefits of implemented eHealth solutions at ten European sites. (eHealth IMPACT project study supported by the European Commission Information Society and Media Directorate-General.). 2006, European Commission Information Society and Media Directorate-General: Brussels.

85. National IT Institute for Healthcare, Corporate Profile, NICTIZ.

86. Zavod za zdravstveno zavarovanje Slovenije. Elektronske storitve ZZZS. 2008 [cited 2008 28-09-2008]; Available from: http://www.zzzs.si/.

87. Health Insurance Institute of Slovenia (2008) Implementing eHealth solutions in Slovenia for the benefit of all.

88. Trcek, D., et al., Slovene smart card and IP based health-care information system infrastructure. International Journal of Medical Informatics, 2001. 61(1): p. 33-43.

89. Slovenia welcomes new e-Health insurance cards. Card Technology Today, 2008. 20(3): p. 3-4.

90. Gemalto Delivers New Generation e-Health Insurance Cards Solution to Slovenia. 2008 [cited 2009 Sept]; Available from: http://www.smartcardalliance.org/articles/2008/02/19/gemalto-delivers-newgeneration-e-health-insurance-cards-solution-to-slovenia.

91. European Commission, eGovernment Factsheet - Sweden. 2008.

92. Bureau of National Health Insurance, NHI Profile. 2008.

93. Liu, C.T., et al., The impacts of smart cards on hospital information systems - An investigation of the first phase of the National Health Insurance smart card project in Taiwan. International Journal of Medical Informatics, 2006. 75(2): p. 173-181.

94. Bureau of National Health Insurance, National Health Insurance in Taiwan-Profile 2007 Current Status. 2007. 
95. Reiser, S.J., The clinical record in medicine. Part 1: Learning from cases. Ann Intern Med, 1991. 114(10): p. 902-7.

96. Shortliffe, E.H. and J.J. Cimino, Biomedical Informatics: Computer Applications in Health Care and Biomedicine. 3rd ed. 2006: Springer. 1037.

97. Galloway, G., Getting personal. The personal health record is a key element in attaining the complete patient medical record. Healthc Inform, 2006. 23(11): p. 45-6.

98. Wideman, C. and J. Gallet, Analog to digital workflow improvement: a quantitative study. J Digit Imaging, 2006. 19 Suppl 1: p. 29-34.

99. Cruz-Correia, R., et al., Integration of hospital data using agent technologies - A case study. Al Communications, 2005. 18(3): p. 191-200.

100. Vedvik, E., A. Tjora, and A. Faxvaag, Beyond the EPR: Complementary roles of the hospital-wide electronic health record and clinical departmental systems. BMC Medical Informatics and Decision Making, 2009. 9(1): p. 29.

101. Comité Européen de Normalisation, EN 12251: Health informatics - Secure User Identification for Health Care - Management and Security of Authentication by Passwords. 2004.

102. Comité Européen de Normalisation, EN 13606: Health informatics - Electronic health record communication. 2007.

103. openEHR Foundation. openEHR Foundation. [cited 2009 Sept]; Available from: http://www.openehr.org.

104. Bidgood, W.D., Jr. and S.C. Horii, Introduction to the ACR-NEMA DICOM standard. Radiographics, 1992. 12(2): p. 345-55.

105. American College of Radiology and National Electrical Manufacturers Association, Digital Imaging and Communications in Medicine (DICOM) in Part 1: Introduction and Overview. 2007, NEMA: Virginia.

106. Health Level Seven inc. Health Level Seven Homepage. 2009 [cited 2009 03/02]; Available from: http://www.hl7.org/.

107. International Organization for Standardization. ISO/TC215 Homepage. 2009 [cited 2009 03/02]; Available from: http://isotc.iso.org/livelink/livelink?func=II\&objld=529137\&objAction=browse\&sort=na me.

108. Eichelberg, M., et al., A survey and analysis of Electronic Healthcare Record standards. Acm Computing Surveys, 2005. 37(4): p. 277-315.

109. Garde, S., et al. Archetype-based knowledge management for semantic interoperability of electronic health records. 2009.

110. Leslie, H., International developments in openEHR archetypes and templates. Health Information Management Journal, 2008. 37(1): p. 38-39.

111. Hall, M.A. and K.A. Schulman, Ownership of Medical Information. JAMA, 2009. 301(12): p. 1282-1284.

112. Assembleia da República, Lei n.o 67/98 - Protecção de Dados Pessoais. 1998: Diário da República. p. 5536-46.

113. Assembleia da República, Lei 46/2007 - Acesso aos Documentos Administrativos e a sua Reutilização. 2007: Diário da República. p. 5680-86. 
114. Conselho Nacional de Ética Para as Ciências da Vida, Parecer N.o 57: Parecer Sobre o Projecto Lei $n \circ 788 / X$ - "Direitos dos Doentes à Informação e ao Consentimento Informado". 2009.

115. Brailer, D.J., Interoperability: the key to the future health care system. Health Aff (Millwood), 2005. Suppl Web Exclusives: p. W5-19-W5-21.

116. Gunter, T.D. and N.P. Terry, The Emergence of National Electronic Health Record Architectures in the United States and Australia: Models, Costs, and Questions. Journal of Medical Internet Research, 2005. 7(1): p. e3.

117. Jha, A.K., et al., The use of health information technology in seven nations. International Journal of Medical Informatics, 2008. 77(12): p. 848-854.

118. Spronk, R. AORTA, the Dutch national infrastructure (whitepaper). 2008 10/01 [cited 2009 03/02]; Available from: http://www.ringholm.de/docs/00980_en.htm.

119. Administração Central do Sistema de Saúde, RSE - Registo de Saúde Electrónico - R1: Documento de Estado da Arte (public discussion preliminary document). 2009.

120. Santos, R., et al. Registos de Saúde Pessoais: Ainda é cedo para o futuro? in Novas Perspectivas em Sistemas e Tecnologias de Informação. 2007. Porto: Edições Universidade Fernando Pessoa.

121. Grupo de Inteligência Artificial. A Informática na Saúde: O processo clínico electrónico. Sistemas de apoio ao diagnóstico em imagem médica. Integração de sistemas. 2005 [cited 2009 Sept]; Available from: http://gia1.di.uminho.pt/gia/default.aspx?m=50\&t=5001\&d=0.

122. Biostatistics and Medical informatics. VCIntegrator. 20092009 [cited 2009 Sept]; Available from: http://sbim.med.up.pt/index.php?option=com_jresearch\&view=project\&id=70\&task=sh ow\&ltemid=396\&lang=en.

123. Scott, J.T., et al., Kaiser Permanente's experience of implementing an electronic medical record: a qualitative study. BMJ, 2005. 331(7528): p. 1313-6.

124. Zhou, Y.Y., et al., Patient access to an electronic health record with secure messaging: impact on primary care utilization. Am J Manag Care, 2007. 13(7): p. 418-24.

125. Kaiser Permanente. Press release: Kaiser Permanente Completes Implementation of Outpatient EHR. Kaiser Permanente Press Releases: National 2008 May 05; Available from: http://xnet.kp.org/newscenter/pressreleases/nat/nat_080505_hc.html.

126. UK National Health System. NHS Connecting For Health. 2007 10/09/2007 [cited 2008 11/12/2008]; Available from: http://www.connectingforhealth.nhs.uk/.

127. UK National Health System. NHS Healthspace Homepage. 2009 [cited 2009 04/02]; Available from: https://www.healthspace.nhs.uk/visitor/default.aspx.

128. Berk, M., et al., To $E$ or not to E? The case for electronic health records. Acta Neuropsychiatrica, 2008(20): p. 104-106.

129. Chaudhry, B., et al., Systematic Review: Impact of Health Information Technology on Quality, Efficiency, and Costs of Medical Care. Ann Intern Med, 2006. 144(10): p. 742752.

130. Delpierre, C., et al., A systematic review of computer-based patient record systems and quality of care: more randomized clinical trials or a broader approach? Int J Qual Health Care, 2004. 16(5): p. 407-16. 
131. Kaiser Permanente, More Americans Aware of Digital Health Options; Use of Health IT Increases, in Kaiser Permanente Press Releases: National. 2008.

132. Markle Foundation. Attitudes of Americans Regarding Personal Health Records. 2005 [cited 2009 04/02]; Available from: http://www.phrconference.org/assets/research_release_101105.pdf.

133. Corrigan, J., et al., Crossing the Quality Chasm: A New Health System for the 21st Century. 2001, Washington, DC: National Academy Press. 166.

134. Walker, J., et al., The value of health care information exchange and interoperability. Health Aff (Millwood), 2005. Suppl Web Exclusives: p. W5-10-W5-18.

135. Terry, N.P., An eHealth diptych: the impact of privacy regulation on medical error and malpractice litigation. Am J Law Med, 2001. 27(4): p. 361-419.

136. Lohr, S., Health Care Technology Is a Promise Unfinanced, in New York Times. 2004: New York.

137. Markle Foundation, THE COMMON FRAMEWORK: Overview and Principles. 2006.

138. Markle Foundation, Linking Health Care Information: Proposed Methods for Improving Care and Protecting Privacy, in Conecting for Health. 2005.

139. Markle Foundation, Conecting For Health:The Personal Health Working Group. Final Report. 2003.

140. Leslie, H., OpenEHR: the world's record, in Pulse+IT. 2007, Pulse Magazine: Australia. p. 50-55.

141. AHIMA e-HIM Personal Health Record Work Group, The Role of the Personal Health Record in the EHR. Journal of AHIMA, 2005. 76(7): p. 64A-D.

142. Telemedical.com inc. Telemedical.com Homepage. 2009 19/01/2009 [cited 2009 05/02]; Available from: www.TeleMedical.com.

143. PersonalMD. PersonalMD Homepage. 2009 [cited 2009 05/02]; Available from: www.PersonalMD.com.

144. Medefile. Medefile Homepage. 20082008 [cited 2009 05/02]; Available from: www.medifile.com.

145. Capmed. Capmed Homepage. 2009 [cited 2009 05/02]; Available from: www.capmed.com.

146. MyMedicalRecords inc. MyMedicalRecords Homepage. 2008 [cited 2009 05/02]; Available from: www.MyMedicalRecords.com.

147. Medikeeper inc. Medikeeper Homepage. [cited 2009 05/02]; Available from: www.medikeeper.com.

148. Vimsystems. Vital Information Management Systems Homepage. 2007 [cited 2009 05/02]; Available from: http://vim.aediasoft.com/.

149. Sittig, D.F., Personal health records on the internet: a snapshot of the pioneers at the end of the 20th Century. Int J Med Inform, 2002. 65(1): p. 1-6.

150. Kim, M.I. and K.B. Johnson, Personal health records: evaluation of functionality and utility. J Am Med Inform Assoc, 2002. 9(2): p. 171-80.

151. Google. Google health quick tour. Google health 2009 [cited 2009 29/01/2009]; Available from: https://www.google.com/health/html/tour/index.html. 
152. Wangsness, L., Electronic health records raise doubt, in The Boston Globe. 2009, Globe Newspaper: Boston.

153. Microsoft. HealthVault Beta. Microsoft HealthVault 2009 [cited 2009 29/01/2009]; Available from: http://www.healthvault.com/.

154. Hellander, I., The deepening crisis in U.S. health care: a review of data, Spring 2008. Int J Health Serv, 2008. 38(4): p. 607-23.

155. Madrigal, A., Google Launches Medical-Records App, in Wired Blog Network, D. Tweney, Editor. 2008.

156. Part one: National ID - Europe. Biometric Technology Today, 2005. 13(9): p. 10-11.

157. (2006) Relatório Melhores Práticas Mundiais. Cartão de Cidadão.

158. Assembleia da República, Lei no 7/2007 de 5 de Fevereiro: Cria o cartão de cidadão e rege a sua emissão e utilização. 2007: Diário da República. p. 940-948.

159. Assembleia da República, Portaria no 202/2007 de 13 de Fevereiro - Elementos de segurança. 2007: Diário da República.

160. Unidade de Coordenação para a Modernização Administrativa (2007) Manual técnico do Middleware Cartão do Cidadão. Cartão Cidadão.

161. Infineon technologies, SLE66CX680PE / m1534-a13 evaluation documentation v1.2. 2005.

162. Unidade de Coordenação para a Modernização Administrativa (2007) Autenticação com o Cartão de Cidadão. Cartão Cidadão.

163. Agência para a Modernização Administrativa, Controlo do № de Versão do Documento. 2009: Lisbon.

164. Joint Interpretation Library, Application of Attack Potential to Smartcards v2.1. 2006.

165. Drimer, S. and S.J. Murdoch. Keep your enemies close: Distance bounding against smartcard relay attacks. in 16th USENIX Security Symposium. 2007.

166. Kardas, G. and E.T. Tunali, Design and implementation of a smart card based healthcare information system. Computer Methods and Programs in Biomedicine, 2006. 81(1): p. 66-78.

167. Comissão Nacional Proteç̧ão de Dados, Autorização № 385 /2005. 2005.

168. Relatório Final da Prova de Conceito - Projecto Pegasus. 2006.

169. Yang, Y.J., et al., A smart-card-enabled privacy preserving e-prescription system. leee Transactions on Information Technology in Biomedicine, 2004. 8(1): p. 47-58.

170. Lee, H.K., T. Malkin, and E. Nahum, Cryptographic strength of ssl/tls servers: current and recent practices, in Proceedings of the 7th ACM SIGCOMM conference on Internet measurement. 2007, ACM: San Diego, California, USA.

171. Takeda, H., et al., An assessment of PKI and networked electronic patient record system: lessons learned from real patient data exchange at the platform of OCHIS (Osaka Community Healthcare Information System). International Journal of Medical Informatics, 2004(73): p. 311-316.

172. International Organization for Standardization, ISO 27799, in Health informatics Information security management in health using ISO/IEC 27002. 2008: Switzerland. 
173. International Organization for Standardization, ISO/IEC 27002, in Information technology -- Security techniques -- Code of practice for information security management. 2005.

174. Eddy, D.M. and C.H. Clanton, The art of diagnosis: solving the clinicopathological exercise. N Engl J Med, 1982. 306(21): p. 1263-8.

175. Kassirer, J.P., Diagnostic reasoning. Ann Intern Med, 1989. 110(11): p. 893-900.

176. Berg, M., Implementing information systems in health care organizations: myths and challenges. International Journal of Medical Informatics, 2001. 64(2-3): p. 143-156.

177. Heeks, R., Health information systems: Failure, success and improvisation. International Journal of Medical Informatics, 2006. 75(2): p. 125-137.

178. Ash, J.S., M. Berg, and E. Coiera, Some unintended consequences of information technology in health care: the nature of patient care information system-related errors. Journal of the American Medical Informatics Association, 2004. 11(2): p. 104-112.

179. Assembleia da República, Decreto-Lei n.o 62/2003. 2003: Diário da República. p. 21702185.

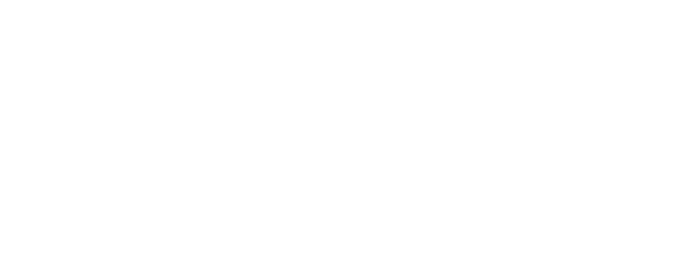

